\title{
WestVirginiaUniversity
}

THE RESEARCH REPOSITORY @ WVU

Graduate Theses, Dissertations, and Problem Reports

2007

\section{Aerosol generation and entrainment model for cough simulations}

Cem Ersahin

West Virginia University

Follow this and additional works at: https://researchrepository.wvu.edu/etd

\section{Recommended Citation}

Ersahin, Cem, "Aerosol generation and entrainment model for cough simulations" (2007). Graduate Theses, Dissertations, and Problem Reports. 2765.

https://researchrepository.wvu.edu/etd/2765

This Dissertation is protected by copyright and/or related rights. It has been brought to you by the The Research Repository @ WVU with permission from the rights-holder(s). You are free to use this Dissertation in any way that is permitted by the copyright and related rights legislation that applies to your use. For other uses you must obtain permission from the rights-holder(s) directly, unless additional rights are indicated by a Creative Commons license in the record and/ or on the work itself. This Dissertation has been accepted for inclusion in WVU Graduate Theses, Dissertations, and Problem Reports collection by an authorized administrator of The Research Repository @ WVU.

For more information, please contact researchrepository@mail.wvu.edu. 


\title{
Aerosol Generation and Entrainment Model for Cough Simulations
}

\author{
Cem Ersahin \\ Dissertation submitted to the \\ College of Engineering and Mineral Resources \\ at West Virginia University \\ in partial fulfillment of the requirements \\ for the degree of \\ Doctor of Philosophy \\ in \\ Mechanical Engineering \\ Dr. Ismail B. Celik, Chair \\ Dr. Ibrahim Yavuz, Co-chair \\ Dr. Omur Cinar Elci \\ Dr. Wade Huebsch \\ Dr. William Lindsley \\ Dr. Andrei Smirnov
}

Mechanical and Aerospace Engineering Department

West Virginia University

Morgantown, West Virginia

2007

Keywords: Aerosol Generation, Cough Simulation, Biofluids, Computational Fluid Dynamics

Copyright 2007 Cem Ersahin 


\section{ABSTRACT}

\section{Aerosol Generation and Entrainment Model for Cough Simulations}

\section{Cem Ersahin}

The airborne transmission of diseases is of great concern to the public health community. The possible spread of infectious disease by aerosols is of particular concern among health-care workers and emergency responders, who face a much greater risk of exposure to these hazards than does the general public. Some diseases, such as influenza, spread by dissemination and inhalation of aerosols of small droplet nuclei that are generated by coughing and remain airborne for an extended time. For that reason a better understanding of the generation of aerosols is important. Therefore, the main objective of this study is to investigate the flow dynamics and the aerosol generation during coughing. This research aims to develop a fairly simple yet an accurate model for the flow simulation in the upper respiratory tract, mainly in the larynx, and the number and size distribution of the aerosols generated during coughing.

In order to provide a more complete analysis tool, a secondary objective is to develop a simple reduced order model for the purpose of simulating the air flow and particle dynamics in the larynx. To this end a 
pseudo two-dimensional model (PTM) has been developed and run for several cases including, sinusoidal laminar and low Reynolds number flow cases including breathing and coughing. The comparison of the PTM model results with FLUENT has shown that the PTM model is capable of producing accurate results within a fraction of execution time needed for the multidimensional FLUENT's model.

The aerosol generation and entrainment model (AGEM) is integrated into this validated one-dimensional model. This is done by utilizing a one dimensional turbulent kinetic energy equation. AGEM is then employed to calculate the aerosol formations during a cough, which is simulated by the one dimensional flow solver. The final size distribution of the aerosol droplets is calculated and these findings are compared with laboratory measurements. It is shown that, with appropriate model coefficients, it is possible to obtain size distribution of aerosols that is consistent with the experimental findings. A parametric study by variation of physical properties of the mucus has also been carried out. The results show some interesting trend with changing surface tension and varying cough signals.

This study may be considered as a step towards a more complete understanding of aerosol generation mechanisms by coughing, which in turn lead to airborne transmission of diseases. The simulation tools developed should serve the scientist to do more parametric studies in a fairly quick manner and investigate the aerosol dispersion in the confined areas as well as studying particle deposition patterns within the upper respiratory track. 


\section{ACKNOWLEDGEMENTS}

I would like to express my sincere appreciation to my advisor Dr. Ismail Celik for his great technical and financial support throughout my studies. Without his support and encouragement, this dissertation would not have been accomplished. Besides his outstanding technical support and guidance, his personal kindheartedness has made those difficult days away from home manageable.

I also would like to thank my committee members for their valuable time reading my dissertation and for their valuable comments. I especially would like to thank Dr. Ibrahim Yavuz for his continuous help as a professor and as a friend during my doctoral study. Also, a special thanks to Dr. Omur Cinar Elci for introducing me to the field of bio-fluids and assisting me with the knowledge of the anatomy of the human respiratory tract. I also appreciate all the help, comments and information I have received from $\mathrm{Dr}$. William Lindsley and Dr. David Frazer when I needed it. I also appreciate the support form NIOSH during my assistantship. 
And the greatest thanks should go to my friends in the Computational Fluid Dynamics and Applied Multi-Physics Center. Their continuous support and friendship made my days in Morgantown very enjoyable and bearable. I especially would like to thank Dr. Pakalapati (Raju) for his great support, friendship-especially on the long weekends-and also for his critical and objective comments, which helped to improve my studies. I also would like to thank Lauren Vogel for her generous help and comments during her busy schedule.

I would like to thank Donna for being there for me during the tough times and her support during my long working days. Also, I cannot skip mentioning my four-legged friends, Köpük, Sir Tuggs, Lucky and the others, who take away all my stress and bring joy to my life after long working hours.

Finally, I would like to thank my parents for their unconditional love and support during every stage of my life. I owe them so much. 


\section{TABLE OF CONTENTS}

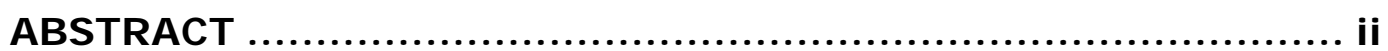

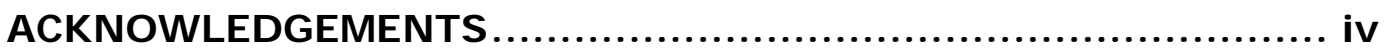

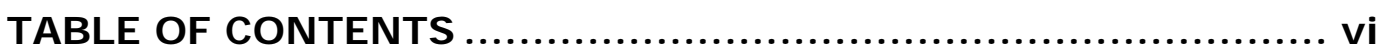

LIST OF FI GURES...........................................................viii

LI ST OF TABLES................................................................

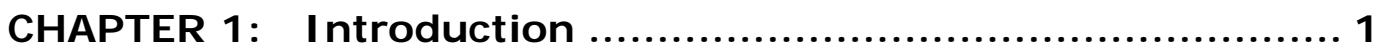

CHAPTER 2: Literature Review ........................................ 5

2.1 Flow Dynamics in Upper Respiratory Tract .................... 5

2.2 Particle Generation Studies ................................. 10

CHAPTER 3: Computational Model ................................... 13

3.1 Pseudo Two-Dimensional Flow Model.......................... 13

3.1.1 One-Dimensional Model ................................... 13

3.1.2 Two-Dimensional Velocity Field Reconstruction ............ 16

3.2 Droplet Model .............................................. 18

3.2.1 Droplet Entrainment Model .............................. 18

3.2.2 Droplet Generation Model.................................. 25

3.2.3 Droplet Breakup Model.................................... 26

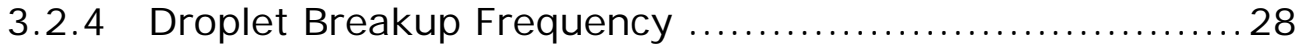

3.2.5 Turbulent Kinetic Energy Equation ....................... 29

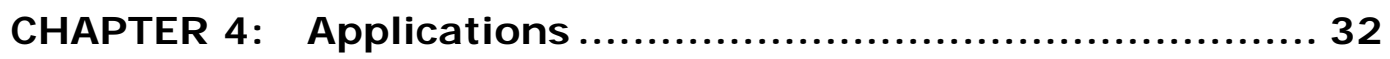

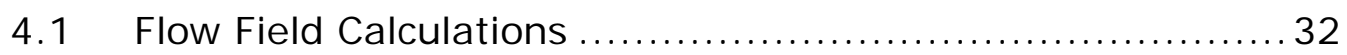

4.1.1 Laminar Flow Case ...................................... 34

4.1.2 Low Reynolds Number Turbulent Flow Case ............... 40

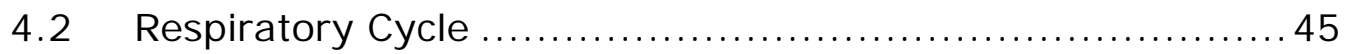




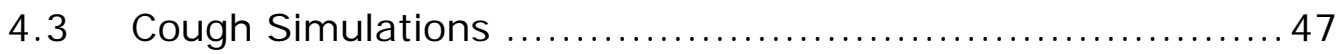

4.3.1 One-Dimensional Flow Analysis .......................... 47

4.3.2 Three-Dimensional Flow Analysis......................... 51

4.4 Kinetic Energy Calculations ................................. 57

4.5 Aerosol Generation ........................................ 59

4.6 Consecutive Coughing ..................................... 69

4.7 Aerosol Generation during a Child's Cough .................... 72

CHAPTER 5: Conclusions and recommendations ................ 77

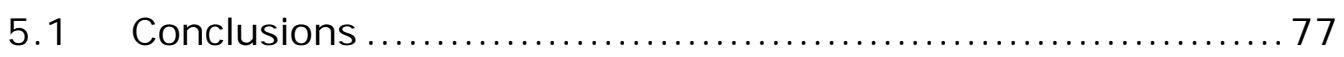

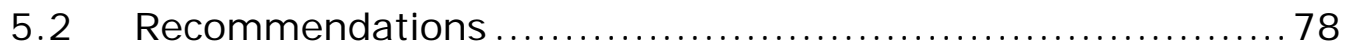

REFERENCES.................................................................... 80

APPENDI X A $\ldots \ldots \ldots \ldots \ldots$

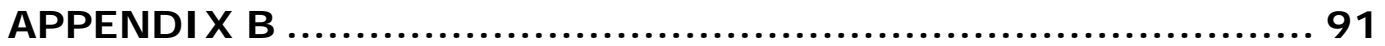

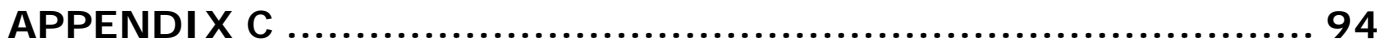




\section{ST OF FI GURES}

Figure 2.1: Schematic sections of the human larynx [9]. ...................6

Figure 2.2: The right half of the larynx in midsagittal section showing the cartilages and the vestibular and vocal ligaments [11] ...........6 6

Figure 2.3: Variation of deposition fraction with aerodynamic diameter

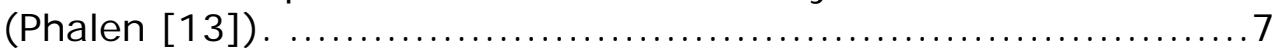

Figure 2.4: Normalized break-up frequency $\left(\mathrm{g} / \mathrm{g}_{\max }, g_{\max }=1769\right)$ with respect to normalized bubble diameter $\left(D / D_{c}\right) \ldots \ldots \ldots \ldots \ldots \ldots 12$

Figure 3.1: Schematic representation of droplet entrainment model. ........ 20

Figure 3.2: Plot of entrainment versus shear stress, Eqn. 3-18. ............ 23

Figure 3.3: Schematic representation of droplet generation model. .......... 25

Figure 3.4: Schematic representation of binary droplet breakup model. .... 27

Figure 4.1: Geometry of the system used in the simulations................ 33

Figure 4.2: Sinusoidal pressure variation at the boundaries................. 34

Figure 4.3: Mass flowrate variation in one complete cycle for laminar case. 35

Figure 4.4: . Axial velocity variation at centerline with time along axial direction for laminar case. ...................................... 35

Figure 4.5: Pressure variation with time along axial direction for laminar case by (a) present model and (b) FLUENT. ......................... 36

Figure 4.6: Velocity profiles at point B for laminar case by (a) present model and (b) FLUENT.

Figure 4.7: Velocity profiles at point C for laminar case by (a) present model and (b) FLUENT.

Figure 4.8: Velocity profiles at point $D$ for laminar case by (a) present model and (b) FLUENT.

Figure 4.9: Mass flowrate variation in one complete cycle for low Reynolds number turbulent flow case.

Figure 4.10: Pressure variation with time along axial direction for low Reynolds number turbulent case by (a) present model and (b) 
FLUENT.

Figure 4.11: Velocity profiles at point B for low Reynolds number turbulent case by (a) present model and (b) FLUENT.

Figure 4.12: Velocity profiles at point $C$ for low Reynolds number turbulent case by (a) present model and (b) FLUENT.

Figure 4.13: Velocity profiles at point $D$ for low Reynolds number turbulent case by (a) present model and (b) FLUENT. ......................44

Figure 4.14: Realistic pressure variation at the boundaries.................. 45

Figure 4.15: Mass flowrate variation in one complete cycle for breathing case.

Figure 4.16: Axial velocity variation at centerline with time along axial direction for breathing case.

Figure 4.17: Coughing data obtained from NIOSH [5] $\ldots \ldots \ldots \ldots \ldots \ldots \ldots \ldots . \ldots \ldots$

Figure 4.18: Extracted coughing data obtained from $\mathrm{NIOSH} . \ldots \ldots \ldots \ldots \ldots 48$

Figure 4.19: Pressure boundary conditions for cough simulations. ........... 49

Figure 4.20: Radius versus axial direction............................... 50

Figure 4.21: Pressure versus axial direction during cough at time $=0.2$ seconds after the cough begins. ............................... 50

Figure 4.22: Axial mean velocity versus axial direction during cough at time $=0.2$ seconds after the cough begins. ..................... 51

Figure 4.23: 200K Mesh generated by Gambit for FLUENT................... 51

Figure 4.24: Pressure contours at the peak of the cough.................... 53

Figure 4.25: Velocity contours at the peak of the cough.................... 53

Figure 4.26: Velocity contours and streamlines at the peak of the cough....53

Figure 4.27: Velocity contour and velocity vectors at the peak of the cough

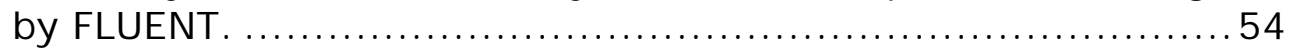

Figure 4.28: Turbulent kinetic energy at the peak of the cough by FLUENT. 54

Figure 4.29: Streamlines with velocity contours at the given axial locations.

Figure 4.30: Comparison of turbulent kinetic energy obtained from the model and FLUENT at a) $\mathrm{Re}=2400$; and b) $\mathrm{Re}=3800 \ldots \ldots \ldots \ldots \ldots \ldots$

Figure 4.31: Measured averaged aerosol size distribution during cough (The vertical axis is the concentration of aerosol) [5] ..................59 59

Figure 4.32: Averaged aerosol size distribution during a cough. .............60

Figure 4.33: Aerosol size distribution during, (a) second cough of first subject; (b) first cough of fourth subject; (c) third cough of fifth subject. 
Figure 4.34: Aerosol size distribution for all three coughs....

Figure 4.35: Difference between the aerosol size distribution for all three coughs and the average size distribution.

Figure 4.36: Effect of random function on the same cough signal. 64

Figure 4.37: Difference between the aerosol size distribution for the same cough in different runs. .65

Figure 4.38: Aerosol size distribution during first cough of third subject. ... 66

Figure 4.39: Aerosol size distribution for various surface tension values. (a)

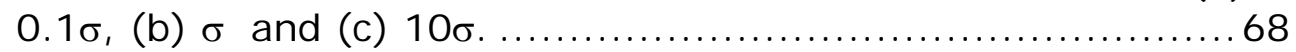

Figure 4.40: Boundary condition for consecutive coughs (same strength)...69

Figure 4.41: Boundary condition for consecutive coughs (decreasing strength). 70

Figure 4.42: Accumulated aerosol size distribution after coughs (same strength).

Figure 4.43: Accumulated aerosol size distribution after coughs (decreasing strength).

Figure 4.44: Upper respiratory tract of a child [55]...................... 73

Figure 4.45: One-dimensional geometry for child's respiratory tract. ....... 73

Figure 4.46: Axial velocity variation at the peak of the cough. ................ 74

Figure 4.47: Variation of flowrate with time. ............................... 74

Figure 4.48: Averaged aerosol size distribution during a child's cough....... 75

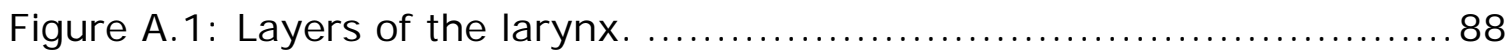

Figure A.2: Distribution of deposited particles among the sub-regions....... 89

Figure A.7: Sediment transport curve, Colorado River, Arizona [40]. ......... 91

Figure A.8: Total entrainment versus velocity in the trachea.................92 


\section{ST OF TABLES}

Table 2.1. Experimental parameters of Martinez-Bazan et al................. 11

Table 3.1. Variables used in droplet entrainment model (DEM) . .............. 21

Table 3.2. Variables used in critical shear stress calculation................... 24

Table 4.1. Physical properties, of the particles injected. .................... 56

Table 4.2. Injection properties. ........................................ 56

Table 4.3. Results of particle tracking from FLUENT simulations. ............. 56

Table 4.4. Model coefficients used in turbulent kinetic energy equation. .... 57 


\section{CHAPTER 1: \\ I NTRODUCTI ON}

"Science is the most reliable guide in life", M.K. Ataturk

The main goal of this study is to develop computational tools to investigate the flow dynamics and the aerosol generation during coughing. This research aims to develop a fairly simple yet an accurate model for the flow simulation in the upper respiratory tract, mainly in the larynx, and the number and size distribution of the aerosols generated during coughing.

Extend of this study will constitute investigation of the interrelationship between aerosol particles and spread of contiguous disease among the population, and also possible diseases such as larynx cancer in the human upper respiratory tract. The study was conducted using computational models that were validated against available experimental and numerical data in the literature $[1,2]$. The experiments conducted at the National Institute for Occupational Safety and Health ( $\mathrm{NIOSH}$ ) $[3,4,5,6]$ was used for the validation purpose of the numerical model. The secondary objective of this study is to compute the particle deposition patterns in the larynx as a function of air flow and geometry of the larynx. The larynx is selected because it is believed to be the most critical sections in the upper human airways. This section also plays a significant role in aerosol generation by cough. Moreover, significant factors that may increase the risk of such diseases could be related to size, shape and density of these aerosol particles, as well as their composition. A particular question is whether the 
high risk is affected more by the amount of deposition or by the physicochemical characteristics of the particles.

Both multi-dimensional Computational Fluid Dynamics (CFD) models such as FLUENT (FLUENT, Inc., Lebanon, NH) and simplified lumped models (e.g. transient pseudo two-dimensional model, PTM) are used. The latter is necessary for two reasons; i) to be able to include compliant walls, i.e. area change as a function of time in the respiratory tract especially near the glottis, ii) to be able to study the effect of many parameters in a reasonable amount of time. It should be noted that multi-dimensional transient models consume extensive amount of execution time.

Although this study is limited to the simulation in the larynx, it is possible to extend the methodology to include other parts of the respiratory system. This will give the opportunity to obtain more detailed and more realistic simulation results which will be combined together with similar studies, such as, aerosol transmission of infectious diseases during coughing or sneezing; prediction and optimization of inhaled therapies, and entire dynamic aerosol transport processes, and drug delivery procedures. Also in this study, the source for the aerosols during a cough is assumed to be the laryngeal part of the upper respiratory tract. It should be noted that in a real cough the main source of the aerosols is from the bronchi and only a smaller portion is from the rest of the respiratory tract.

In order to create a time efficient yet accurate tool, (especially, when a parametric study is in question), there should be an additional simplified model. Due to the unique shape of the larynx, the geometry was kept simple to obtain a practical yet accurate solution. Considering the moving walls and unsteady flow conditions and time consuming aerosol breakup process, the only acceptable approach is believed to be the implementation of simplified physics and geometry in the simulations. The complex system is simplified by 
assuming to be axially symmetric with a reconstructed (not calculated directly) flow field. This approach requires many input parameters from three-dimensional simulations to relate the flow field to the flow conditions and geometry of the system. This method is shown to be accurate enough to produce similar results to those of obtained from a multi-dimensional solver, such as FLUENT, in a fraction of a time $[7,8]$.

In the first phase of the study, the coughing condition in the larynx has been simulated. The coughing conditions were generated by the use of experimental data obtained from $\mathrm{NIOSH}[3,4,5]$. The flowrate versus time data was used to obtain the pressure variation at the lung side of the larynx. The simulations were carried out with the one-dimensional code and FLUENT. While the axial variation of mean velocity, pressure and density was investigated in the one-dimensional code, the velocity field was generated in FLUENT, and particles were introduced. The mucus was entrained during the cough at the subglottis and the number and size distribution of aerosols calculated.

In the second phase of the study the air flow characteristics and particle deposition patterns in the larynx were studied. In this stage a pseudo-two-dimensional transient flow model was employed, where the one dimensional flow was solved and then the two-dimensional flow field was reconstructed with the empirical relations depending on the flow regime. Quartz particles at different sizes were injected into the system and the deposition patterns of the particles were studied during normal breathing conditions [7]. The preliminary findings for the particle deposition in the larynx are given in Appendix A.

The experience gained and the tools generated up to this stage of the study are anticipated to be the stepping stones of the future studies and will help to develop a complete transient model for the flow simulation and 
particle tracking in the anatomically represented larynx and later in the entire upper human respiratory tract. This complete model for the human respiratory tract is aimed to be a practical tool for the medical scientists to simulate different breathing conditions, coughing and sneezing and observe the aerosol generation during cough and spread of these aerosols in the surrounding of the effected person, and particle deposition patterns, and study/examine epidemiological relations with particles, as well as drug transfer to the lungs. 


\section{CHAPTER 2: \\ LITERATURE REVI EW}

"The things I want to know are in books; my best friend is the man who'll get me a book I ain't read", A. Lincoln

\subsection{Flow Dynamics in Upper Respiratory Tract}

The larynx is a special section of the upper respiratory tract and its main role is to create phonation and a protective barrier before the lower respiratory tract (Fig.2.1) [9]. As an organ, the larynx has a unique dynamic feature which cannot be found in the other parts of the human body [10]. The larynx has a three dimensional flexibility and continuous active movements that effect air flow dynamics. The complex muscular structure of the larynx is also shown in Figure 2.2 [11].

Besides smoking and alcohol, occupational exposures play a role in the etiology of laryngeal disorders, especially cancer, which is one of the most common types of cancer in the Eastern Europe and Mediterranean region [12]. It is believed that the tissue interaction with particles suspended in air increase the risk of cancer. It is anticipated that the rate of aerosol generation during cough is proportional to the amount of aerosol deposition during breathing. Therefore, the deposition behavior of solid particulate matter inside the larynx of a human gains a significant importance (Fig.2.3) [13]. Figure 2.3 shows that a significant portion of the particles deposited in the larynx having diameters less than $1 \mu \mathrm{m}$. In the light of this observation it 
is deemed necessary to focus this study on understandings of the air flow characteristics and particle deposition patterns in the larynx.

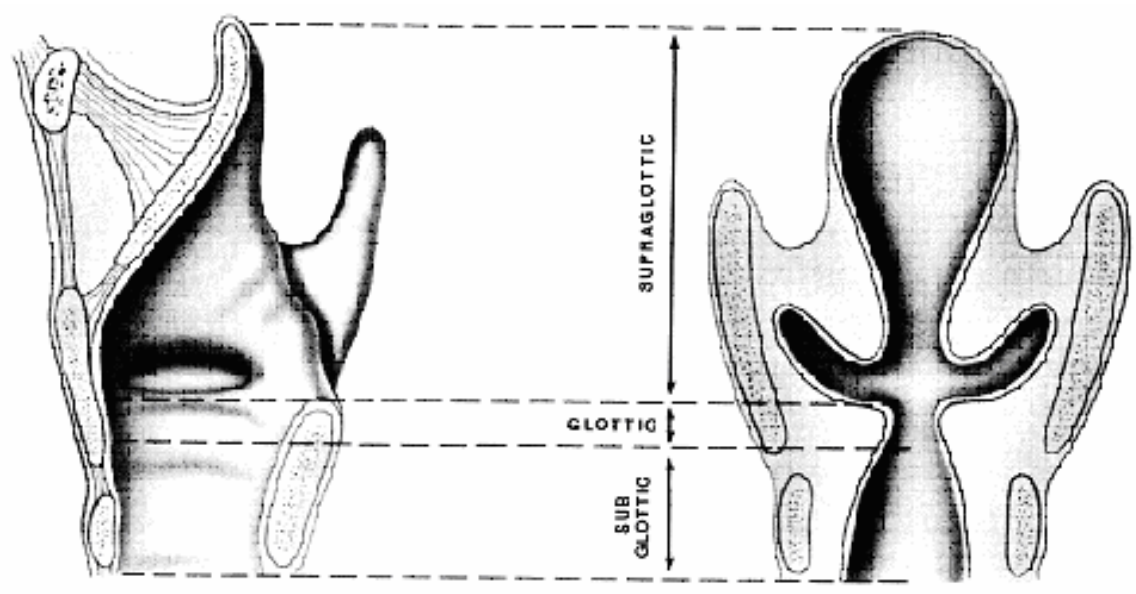

Figure 2.1: Schematic sections of the human larynx [9].

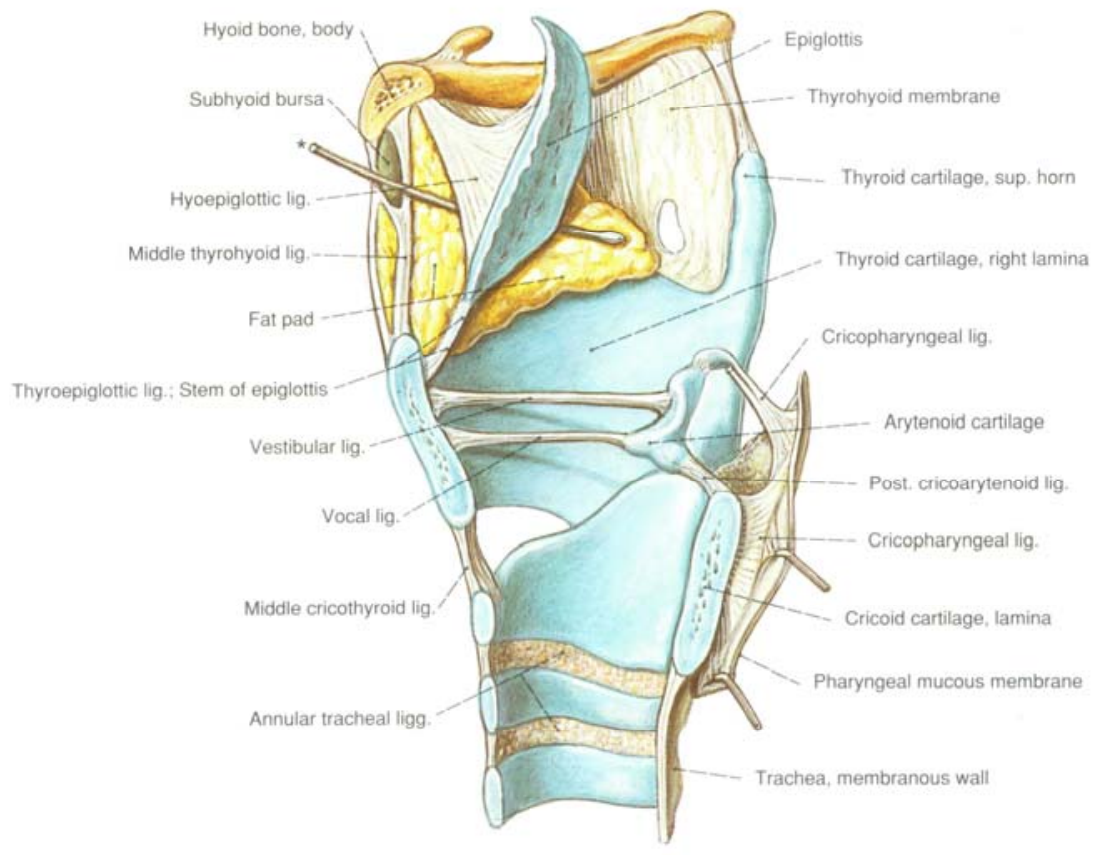

Figure 2.2: The right half of the larynx in midsagittal section showing the cartilages and the vestibular and vocal ligaments [11]. 


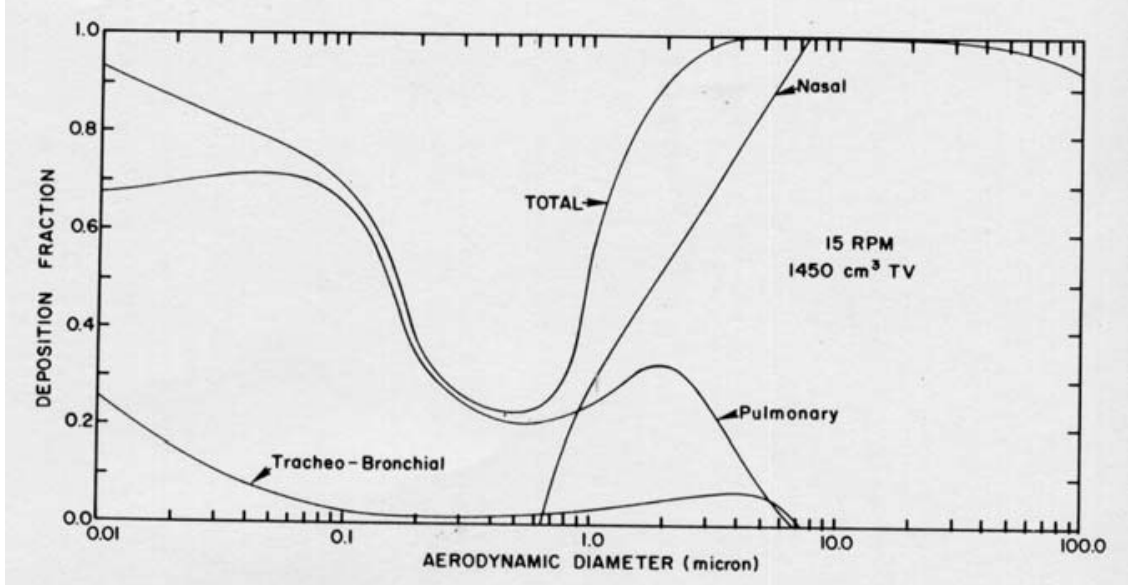

Figure 2.3: Variation of deposition fraction with aerodynamic diameter (Phalen [13]).

Flow simulation and particle tracking within the human respiratory system has important applications to inhalation toxicology and aerosol medicine. Particularly, the larynx has an important role in the development of the flow field in the airways and is prone to one of the most common cancer types due to the particle deposition [14]. Therefore, the distributions of inhaled particles within the larynx enhances the epidemiological concerns $[15,16]$. There have been numerous analytical, experimental and numerical studies concerning air flow and particulate transport processes within the upper airways and the larynx (See [7] for a review). Previous studies reported that occupational exposure to particulate matter, including silica, cotton dust, PAH and diesel exhaust, played a role in the etiology of laryngeal cancer $[17,18]$.

One of the difficulties encountered in modeling of the larynx is its moving boundaries. The transient nature of the respiratory system makes the complete simulation of the larynx very difficult, which results in two classes of studies in the literature; (1) steady flow simulations with moving boundaries decoupled from the transients and transient simulations with nonmoving boundaries; (2) reduced lumped models focusing on the transients. 
The proposed methodology is a hybrid of these two approaches and is capable of handling moving-boundaries. The pseudo two-dimensional nature of the method makes the simulation of transient flows more robust and allows for extensive parametric study. Hence, it will also be possible to capture the wave mechanics in the system under the transient flow conditions. The same method can also be adapted to compressible flows, which may be required in extreme cases where the glottal opening is nearly or completely closed.

Accurate description of the flow field in the larynx is the prerequisite step for the particle tracking and calculating the deposition behavior of the particles. After determining the flow field as a function of time and space in a complete cycle, the particles can be introduced into the system during the inhalation. These particles can then be traced and the deposition locations recorded. This in turn can be used to calculate histograms and probabilities of deposition in specific regions in the larynx. In what follows the proposed methodology for studying the particle deposition pattern in the larynx under various conditions is briefly described.

There have been numerous experimental, numerical and theoretical studies focusing on the human upper respiratory track and especially on the larynx to understand the flow dynamics and particle deposition patterns [1316,19-23]. These studies reveal the flow dynamics in the respiratory tract and are used to better understand the detailed mechanisms involved. However most of these studies exclude the exhalation part of the breathing cycle from the inhalation part, or the inhalation is taken into consideration as a constant flow through the system, or the geometry of the respiratory tract is assumed to be a circular pipe. In the current study the geometry changes and transient flow is taken into consideration. Moreover, the inhalation part of the breathing cycle is not excluded from the exhalation. 
There are different flow regimes present in the flow through the larynx. The flow upstream of the glottis (supraglottic during inhaling and subglottic during exhaling) can be considered laminar, whereas the flow downstream (subglottic during inhaling and supraglottic during exhaling) of the glottis is turbulent and requires accurate turbulence modeling [19]. Although, there are opposing views like Ertbruggen et al. [20], that the flow is laminar throughout the larynx, there are reasons to believe that the former to be true from our own experience in this subject [7]. This can also be seen from the numerical simulation of the flow during coughing conditions [8]. This view is also supported by the study of Alipour et al. [19], Gemci et al. [21, 22], and Renotte et al. [23]. To be able to account for this complex flow regimes a widely accepted and validated CFD code, namely FLUENT, is used in conjunction with a pseudo two-dimensional code, which was developed in house. As to our knowledge, there is no attempt in implementing moving boundaries to the study of the flow in the larynx. There are other studies on this subject using the KIVA code $[20,21]$. Although KIVA is very capable of solving a problem like this $[24,25,26]$, the lack of documentation and the pre- and post-processing modules, has discouraged the current researcher in using this code. Some researchers also used the FLUENT code to tackle this problem. However, even in this case they only investigated several instances of the larynx geometry, separately [23].

As it can be seen from the literature there are many studies on the flow field and particle deposition patterns in the upper respiratory tract in detail. However, each one of these studies is concentrated on one portion of the whole picture and neglects the rest of the picture due to the very complex nature of the system. As it is explained before, the geometry of the larynx is very complex and moreover moving boundaries during the normal operating conditions makes it even more problematical to model. The transient and reciprocating/pulsative behavior of the flow is another challenging issue in the complex geometry of the respiratory system. This study is an attempt to alleviate some of the short comings of previous 
studies by including inhaling and exhaling as well as coughing processes. Moreover a simple one-dimensional model is developed that can be used easily for parametric study hence for diagnostics of certain diseases.

In addition to the flow simulation in the respiratory tract and aerosol generation model for the cough a preliminary study was conducted to see the behavior of the deposition of particles under various breathing condition. For particle tracking a subroutine, which is previously developed by $\mathrm{Hu}$ [37], is included into the pseudo two-dimensional model. In this approach the Lagrangian method is used, where each particle or parcel is tracked

individually and the particle deposition on the larynx wall is recorded, which is believed to be an important parameter in the occurance of laryngeal cancer. The turbulence model of choice in this research is the low-Re $k-\varepsilon$ RNG model, which seems to be a better alternative than the standard $k-\varepsilon$ or RNG $k-\varepsilon$ models according to Yavuz et al. [24], as used in previous studies $[19,20]$.

\subsection{Particle Generation Studies}

There have been numerous experimental studies in the literature to model the break-up frequency of large bubbles or drops since late 1940's [27]. In many of these experiments the measurements of the time evolution of the drop size were taken in stirred tanks or turbulent pipe flows $[28,29,30]$. However, the difficulty in the stirred tank model is that the turbulence cannot be characterized very well due to the inhomogeneity in the turbulence arising from the presence of impeller blades.

Martinez-Bazan et al. [31,32], have utilized a completely different setup than the previous researchers in order to minimize the inhomogeneity in the turbulence and to satisfy the isotropy condition in the turbulence. They injected air bubbles into a water jet at the same mean velocity so that only 
the turbulence would affect the break up process. They have developed a model for the frequency $(\mathrm{g})$ of the break-up process as a function of the original bubble diameter (D) and dissipation rate $(\varepsilon)$ (Eqn. 2-1).

$$
g(\varepsilon, D)=K_{g} \frac{\sqrt{\beta(\varepsilon D)^{2 / 3}-\frac{12 \sigma}{\rho D}}}{D}
$$

where $\mathrm{K}_{\mathrm{g}}$ and $\beta$ are empirical constants, and $\sigma$ is the surface tension. The plot of this equation can be seen in Figure 2.4 for their experimental conditions (See Table 2.1). The relation given in Equation 2-1 is used in the present model to calculate the break up frequency of each droplet. The selection of this relation is made due to the well defined final form of the breakup frequency relation for this type of breakup process in addition to the experimental conditions. As explained earlier, the experiment is set up in such a way that only effect of turbulence is taken into consideration. This direct relation of turbulence and the breakup frequency is proper in this present approach since the effect of flow is transferred to the aerosol generation and entrainment models through the solution of turbulent kinetic energy.

Table 2.1. Experimental parameters of Martinez-Bazan et al.

\begin{tabular}{ccc} 
Variable & Value & Unit \\
\hline \hline$\beta$ & 8.2 & - \\
$\mathrm{K}_{\mathrm{g}}$ & 0.25 & - \\
$\sigma$ & 0.5 & $\mathrm{~N} / \mathrm{m}$ \\
$\rho$ & 1.225 & $\mathrm{~kg} / \mathrm{m}^{3}$ \\
$\varepsilon$ & 2000 & $\mathrm{~m}^{2} / \mathrm{s}^{3}$ \\
\hline
\end{tabular}




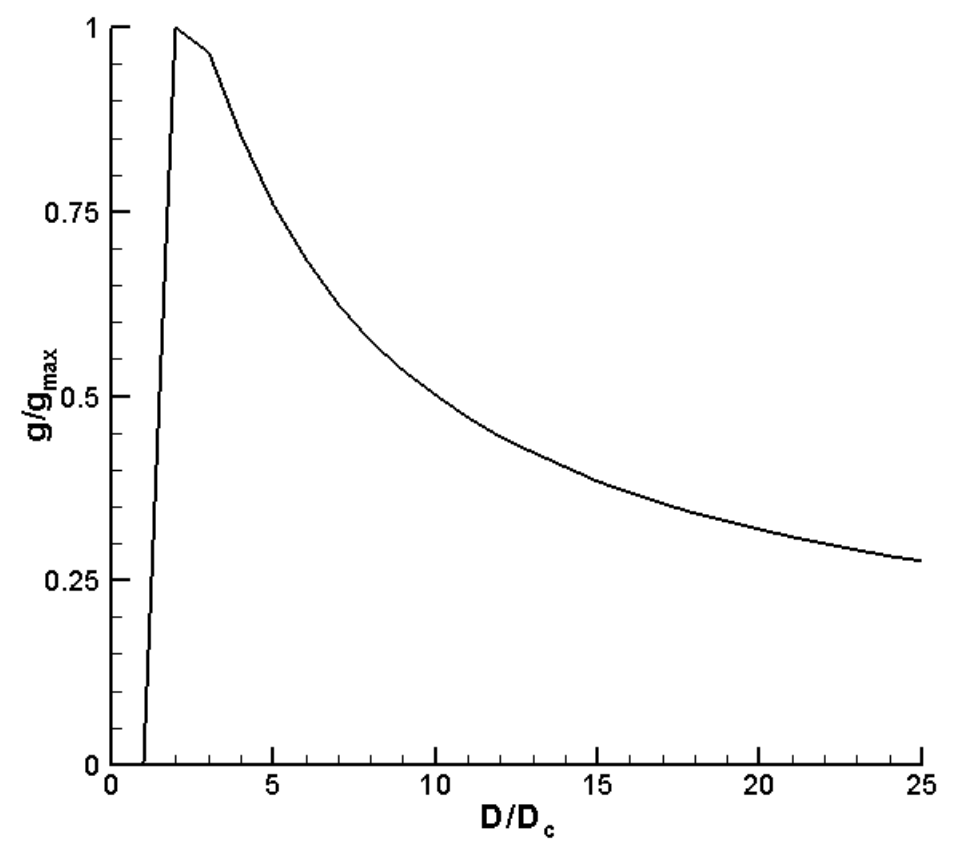

Figure 2.4: Normalized break-up frequency $\left(\mathrm{g} / \mathrm{g}_{\max }, g_{\max }=1769\right)$ with respect to normalized bubble diameter $\left(D / D_{c}\right)$. 


\section{CHAPTER 3: COMPUTATI ONAL MODEL}

"Yes, we have to divide up our time like that, between our politics and our equations. But to me our equations are far more important, for politics are only a matter of present concern. A mathematical equation stands forever", A. Einstein

\subsection{Pseudo Two-Dimensional Flow Model}

In this pseudo two-dimensional study, a simplifying assumption has been made for the three-dimensional flow within the larynx to calculate the average velocity. Although, the actual system is not axisymmetric, for the purpose of obtaining a simple algorithm for estimating the particle distribution in the larynx, an axisymmetric assumption was necessary.

\subsubsection{One-Dimensional Model}

The flow is governed by the continuity (Eqn. 3-1) and momentum equations (Eqn. 3-2), which can be written as follows for a quasi-twodimensional flow.

$$
\begin{aligned}
& \frac{\partial(\rho \mathrm{A})}{\partial \mathrm{t}}=-\frac{\partial \dot{\mathrm{m}}}{\partial \mathrm{x}} \ldots \ldots \ldots \ldots \ldots \ldots \ldots \ldots \ldots \ldots . . . . . . . . \\
& \frac{\partial \dot{\mathrm{m}}}{\partial \mathrm{t}}=-\frac{\partial}{\partial \mathrm{x}}(\mathrm{u} \dot{\mathrm{m}})-\mathrm{A} \frac{\partial \mathrm{P}}{\partial \mathrm{x}}-\frac{1}{2} \mathrm{C}_{\mathrm{f}} \rho|\mathrm{u}| \mathrm{u}
\end{aligned}
$$


where $\dot{m}=\rho \mathrm{uA}$ and $\mathrm{C}_{\mathrm{f}}$ is the friction coefficient which is defined as a function of Reynolds number (Eqn. 3-3). As it can be seen from this relation the friction coefficient consists of three different functions. First function covers the laminar regime, last covers the turbulent regime, and the second is the linear combination of the other two for the transitional regime.

$$
C_{f}=\left\{\begin{array}{cc}
\frac{16}{\operatorname{Re}} & \operatorname{Re}<2000 \\
b & 2000<\operatorname{Re}<4000 \\
\frac{0.316}{\operatorname{Re}^{0.25}} & \operatorname{Re}>4000
\end{array}\right.
$$

where $b=0.0397\left(\frac{\operatorname{Re}-2000}{2000}\right)+0.008\left(1-\frac{\text { Re-2000 }}{2000}\right)$.

We let $\mathrm{F}=\rho \mathrm{Au}=\dot{\mathrm{m}}$ and write the momentum equation as;

$$
\frac{\partial F}{\partial t}=F_{r h s}-A \frac{\partial P}{\partial x}
$$

where $\mathrm{F}_{\text {rhs }}$ represents all the terms involving convection, diffusion, and friction.

\section{Numerical Method}

For the numerical solution of the momentum equation the pressurecorrection method is employed as proposed by MacCormack [34] and implemented by Tatli [1] and Celik et al. [2]. First, an approximate value for $\hat{F}$ is calculated without the influence of pressure. This step is called the predictor step and given as the following (Eqn. 3-5).

$$
\hat{F}=F^{n}+\Delta t F_{\text {rhs }}^{n}
$$


where superscript $\mathrm{n}$ denotes the previous time step.

Then, the correction step is applied (Eqn. 3-6).

$$
\mathrm{F}^{*}=\mathrm{F}^{\mathrm{n}}+\frac{\Delta \mathrm{t}}{2}\left(\mathrm{~F}_{\text {rhs }}^{\mathrm{n}}+\hat{\mathrm{F}}_{\text {rhs }}^{\mathrm{n}}\right)
$$

where $\hat{F}_{\text {rhs }}^{n}$ is the right hand side of Equation 3-4 without the pressure evaluated using $\hat{F}$.

If we had included the pressure we would have had

$$
F^{n+1}=F^{n}+\frac{\Delta t}{2}\left(F_{\text {rhs }}^{n}+\hat{F}_{\text {rhs }}\right)-\Delta t A \frac{\partial P^{n+1}}{\partial x}
$$

Here superscript $\mathrm{n}+1$ denotes the current time level. We subtract Equation 3-6 from Equation 3-7 to obtain;

$$
F^{n+1}-F^{*}=-\Delta t A \frac{\partial P^{n+1}}{\partial x}
$$

We assume that if the pressure field is correct, $F^{n+1}$ should satisfy the continuity equation (Eqn. 3-1), i.e.

$$
\frac{\partial}{\partial \mathbf{x}}\left(\mathrm{F}^{\mathrm{n}+1}\right)=-\frac{\partial(\rho \mathrm{A})}{\partial \mathrm{t}}
$$

Taking the derivative of Equation 3-8 with respect to $\mathrm{x}$ and using Equation 3-9 yields an equation for pressure; 


$$
\frac{\partial}{\partial \mathbf{x}}\left(\mathrm{A} \frac{\partial \mathbf{P}^{\mathrm{n}+1}}{\partial \mathbf{x}}\right)=\frac{1}{\Delta \mathrm{t}}\left[\frac{\partial \hat{\mathrm{F}}^{*}}{\partial \mathbf{x}}+\frac{\partial(\rho \mathrm{A})}{\partial \mathrm{t}}\right]
$$

Equation 3-10 corresponds to the Poisson equation for pressure in quasi-two-dimensional flows. The derivative of the density with respect to time can be approximated explicitly (Eqn. 3-11).

$$
\frac{\partial \rho}{\partial \mathrm{t}}=\frac{\rho^{\mathrm{n}}-\rho^{\mathrm{n}-1}}{\Delta \mathrm{t}}
$$

After the pressure field calculation Equation $3-8$ is used to calculate $F^{n+1}$ from $F^{*}$ field. Equation 3-10 is discretized using the finite volume method (FVM). Equations 3-1 and 3-4 are solved using the MacCormack [35, 36] method. More details of these derivations and application can be found elsewhere $[1,2]$.

\subsubsection{Two-Dimensional Velocity Field Reconstruction}

After calculating the average velocity along the axial direction, a twodimensional velocity field is constructed. An assumed velocity profile has been applied which can handle the recirculating region by using certain empirical coefficients tuned according to the results obtained using FLUENT runs. The parameters used in the velocity profile are defined as functions of Reynolds number.

It has been noted that during the inhalation and exhalation upstream of the glottal opening of the larynx, a laminar velocity profile exists and downstream of the glottal opening, the flow becomes a thin jet around the centerline with a recirculation zone next to it. Considering that the flow changes its direction in a cycle, upstream and downstream switches when the flow is reversed. In order to capture this behavior, two different velocity 
profiles have been applied and selected depending on the direction of the flow. In the upstream region, a parabolic velocity profile (Eq. 3-12) is applied for the axial velocity component. The radial velocity is then related to the angle of the jet besides the axial velocity in such a way that velocity vectors are parallel to the centerline at the centerline and parallel to the jet border as they approach the recirculation region. Here,

$$
\frac{u_{r}}{u_{\max }}=1-\left(\frac{r}{R}\right)^{n}
$$

where $u_{r}$ is the axial velocity at point $r, u_{\max }$ is the maximum velocity at the centerline, $r$ is the distance from the center, $R$ is radius of the larynx, and $\mathrm{n}$ is 2 and 0.1 for, the laminar regime and turbulent regime, respectively. The radial velocity is calculated from

$$
\mathrm{v}_{\mathrm{r}}=\mathrm{u}_{\max } \tan \theta_{\mathrm{R}}\left(\frac{\mathrm{r}}{\mathrm{R}}\right)
$$

where $\theta_{\mathrm{R}}$ is the angle of the wall at the specific axial location at $r=\mathrm{R}$, and $\left(\frac{r}{R} \theta_{R}\right)$ becomes zero at the center $(r=0)$.

Downstream of the glottal opening, Eqs. 3-12 and 3-13 are used in the jet flow where $R$ is replaced by $R_{\text {jet }}$ as the radius of the jet. For the recirculation region, potential flow (Eq. 3-14) has been solved on a transformed domain and mapped back onto the physical domain in such a way that the velocity field corresponds to the stream function $\psi$ determined from $\dot{m}$. 


$$
\nabla^{2} \psi=\psi_{\varepsilon \varepsilon} \varepsilon^{2}+\left(\eta_{\mathrm{x}}^{2}+\eta_{\mathrm{y}}^{2}\right) \psi_{\eta \eta}=-\psi_{\eta} \eta_{\mathrm{xx}}
$$

The velocity components are calculated from $u=\frac{\partial \psi}{\partial y}$ and $v=-\frac{\partial \psi}{\partial x}$; the dimensionless coordinates are defined as $\varepsilon=\frac{\mathrm{x}}{\mathrm{x}_{\mathrm{R}}}$ and $\eta=\frac{\mathrm{r}-\mathrm{R}_{\mathrm{jet}}}{\mathrm{R}-\mathrm{R}_{\mathrm{jet}}}$; and the boundary conditions are defined so that the streamlines follow the boundaries of the recirculation region. Turbulence fluctuations in the magnitude of $10 \%$ of the average velocity are also added on the velocity field in order to imitate the turbulent flow.

Equation for the radius of the jet is calculated from Eq.3-15 by considering the reattachment length $\left(x_{r}\right)$ of the flow, which depends on the Reynolds number of the flow.

$$
\mathrm{R}_{\mathrm{jet}}(\mathrm{x})=\mathrm{R}_{\mathrm{cr}}+\left(\mathrm{R}-\mathrm{R}_{\mathrm{cr}}\right)\left(\frac{\mathrm{x}}{\mathrm{x}_{\mathrm{r}}}\right)^{2}
$$

where $R_{c r}$ is the radius of the glottal opening and $x_{r}$ is the reattachment length. Note that to maintain simplicity we use a two-dimensional stream function on a given $x-r$ plane, then revolve this field around the centerline.

\subsection{Droplet Model}

\subsubsection{Droplet Entrainment Model}

The role of droplet entrainment model (DEM) is to calculate the total amount of mucus (phlegm) to be entrained into the system. This model only 
determines the total amount of mucus entrained into the system, and does not take break-up process and size distribution into consideration.

The wall of the respiratory tract is assumed to be partially covered with mucus at a certain thickness depending on age, gender and health condition of the person. It is assumed that this mucus is locally homogenous. It should be noted that the particles deposited during the breathing process are also be included in this mucus. Therefore physical properties of the mucus depend on the particle content. However, this mixture is taken as a homogenous mixture without considering two-phase substance and effective values for these physical properties are used. The thickness of the mucus and the fraction of the area covered with mucus are two of the unknowns at this stage of the study. Therefore these two parameters should be obtained in further studies and updated as needed.

There is a certain film thickness of the mucus which is held by the surface tension of the mucus. The shear stress on the wall of the larynx can be calculated from the solution of the one-dimensional velocity. In this approach the effect of flow is felt on the mucus layer in terms of kinetic energy. In the case of droplets there is a critical diameter $\left(D_{c}\right)$, at which the shear stress (deformation forces, $\tau$ ) acting on the droplet is equal to the surface tension (confinement forces, $\tau_{c r}$ ) of the droplets. As the deformation force exceeds the confinement forces the droplet breaks up into smaller droplets. The droplets with larger diameter are prone to break-up into daughter droplets [39].

Similarly, a relation for a film may be used, where the critical diameter is replaced by critical thickness, $\delta_{c}$. At this critical thickness the surface tension and the shear stress should be in balance and this is called critical stress and represented by $\tau$. Therefore, it is expected that when the shear stress exceeds the surface tension some of the mucus will be entrained into 
the system until a new balance is attained. The amount of mucus is going to be calculated by this model. A schematic representation of the proposed model is given in Figure 3.1 as a visual representation of DEM. In this figure, $\delta, \tau$ and $\tau_{\mathrm{cr}}$ represent, film thickness of the mucus layer, shear stress on the layer and critical shear stress of the mucus surface, respectively. D is the diameter of the respiratory tract and $v^{\prime}$ and $k$ represent the turbulence flow fluctuations in terms of turbulent kinetic energy.

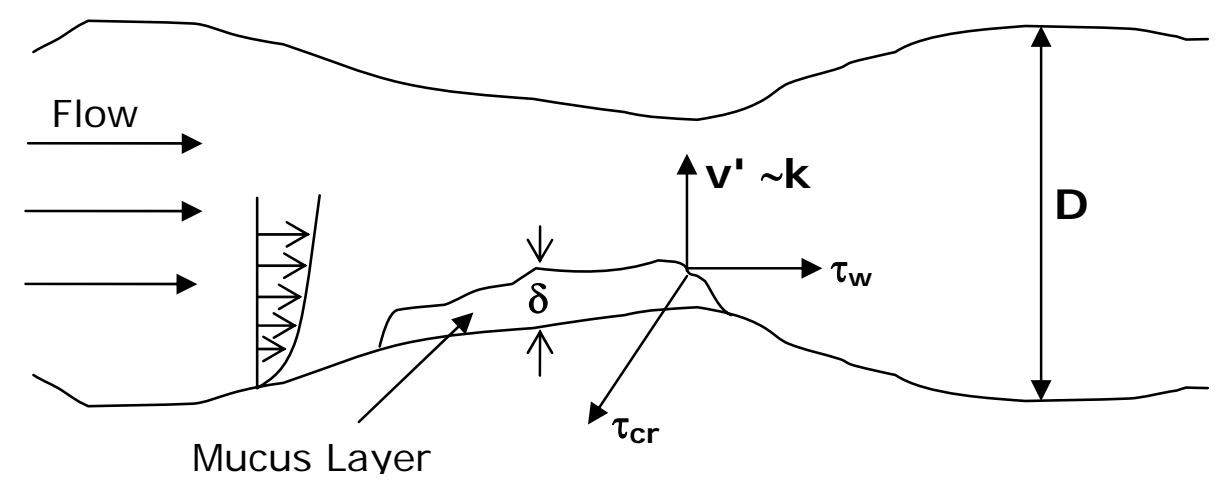

Figure 3.1: Schematic representation of droplet entrainment model.

The aim of this model is to calculate the rate of entrainment amount ( $\dot{E}$ ). For the development of this model it is assumed that the entrainment amount will depend on the physical parameters given in Table 3.1. The $\dot{E}$ is assumed to be a function of characteristic length, entrainment velocity, shear stress and critical shear stress (Eqn. 3-16).

$$
\frac{d E}{d t}=f\left(L_{c h}, V_{e}, \tau_{w}, \tau_{c r}\right)
$$


Table 3.1. Variables used in droplet entrainment model (DEM).

\begin{tabular}{clc} 
Variable & \multicolumn{1}{c}{ Definition } & Unit \\
\hline \hline $\mathrm{E}$ & Entrainment & $\mathrm{m}^{3}$ \\
$\mathrm{D}$ & Diameter & $\mathrm{m}$ \\
$\mathrm{k}_{\max }$ & Turbulent kinetic energy & $\mathrm{m}^{2} / \mathrm{s}^{2}$ \\
$\tau_{\mathrm{w}}$ & Shear stress & $\mathrm{N} / \mathrm{m}^{2}$ \\
$\tau_{\mathrm{cr}}$ & Critical shear stress & $\mathrm{N} / \mathrm{m}^{2}$ \\
$\varepsilon$ & Fraction of the mucus layer & - \\
$C_{\varepsilon}$ & Model constant & - \\
$\mathrm{n}$ & Model constant $(\mathrm{n}=2)$ & - \\
\hline
\end{tabular}

The Laursen formula for sediment discharge, which is used to calculate the total discharge in sediment transportation [40,41], is modified to calculate the entrainment during the cough. The entrainment rate is taken to be proportional to diameter of the respiratory tract (D), turbulent kinetic energy $(k)$, which represents the entrainment velocity, shear stress $\left(\tau_{w}\right)$ and critical shear stress $\left(\tau_{\mathrm{cr}}\right)$. Rearranging these variables and introducing two model constants $\left(C_{\varepsilon}\right.$ and $\left.n\right)$ and a coefficient $(\varepsilon)$ which defines the fraction of area covered with mucus to the inner surface area of the upper respiratory tract, $\dot{E}$ can be expressed as;

$$
\frac{\mathrm{dE}}{\mathrm{dt}}=\mathrm{C}_{\varepsilon} \varepsilon_{\mathrm{A}} \frac{\mathrm{D}^{2}}{2} \sqrt{\mathrm{k}_{\max }}\left(\frac{\tau_{\mathrm{w}}}{\tau_{\mathrm{cr}}}-1\right)^{\mathrm{n}}
$$

Selection of the model coefficients and the unknown physical properties, such as, the fraction of the area covered with mucus, is done in a way that the total amount of the entrainment matches the total amount of the aerosol generated during a cough measured experimentally. In this 
entrainment model, only the net entrainment is taken into consideration. During the entrainment and in the other sub models the deposition of the aerosols on the trachea is neglected. Therefore, the amount of mucus is entrained in a real life would be more than what is calculated with this model, however the difference of the aerosols would be depositing on the respiratory tract before they make it out of the system.

However, by considering the critical diameter concept in the break-up process it should be clear that shear stress should be greater than a certain value $\left(\tau_{\mathrm{cr}}\right)$ and the driving force should be the difference between the shear force and the critical shear force $\left(\tau_{\mathrm{w}}-\tau_{\mathrm{cr}}\right)$. Therefore for numerical application Equation 3-17 is modified to read;

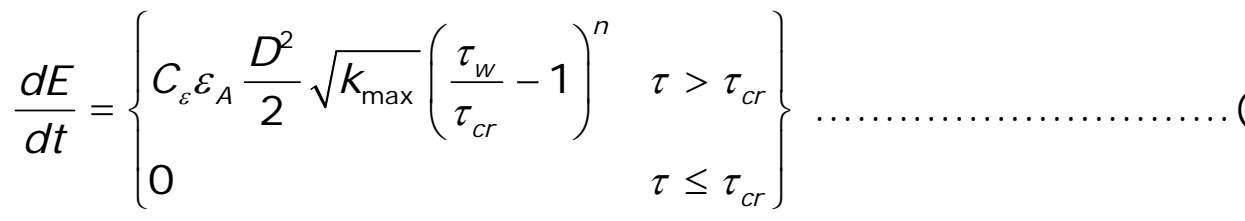

The trend of the entrainment rate versus shear stress is plotted in Figure 3.2 As it is seen from the figure, until the critical shear stress is reached, the entrainment amount remains at zero and after exceeding this value the amount of entrainment increases with increasing shear stress on the mucus.

The wall shear stress $\tau_{\mathrm{w}}$ involved in Eqn. 3-18 can be calculated from;

$$
\tau=-\mu \frac{\partial \mathrm{u}}{\partial \mathrm{n}}
$$




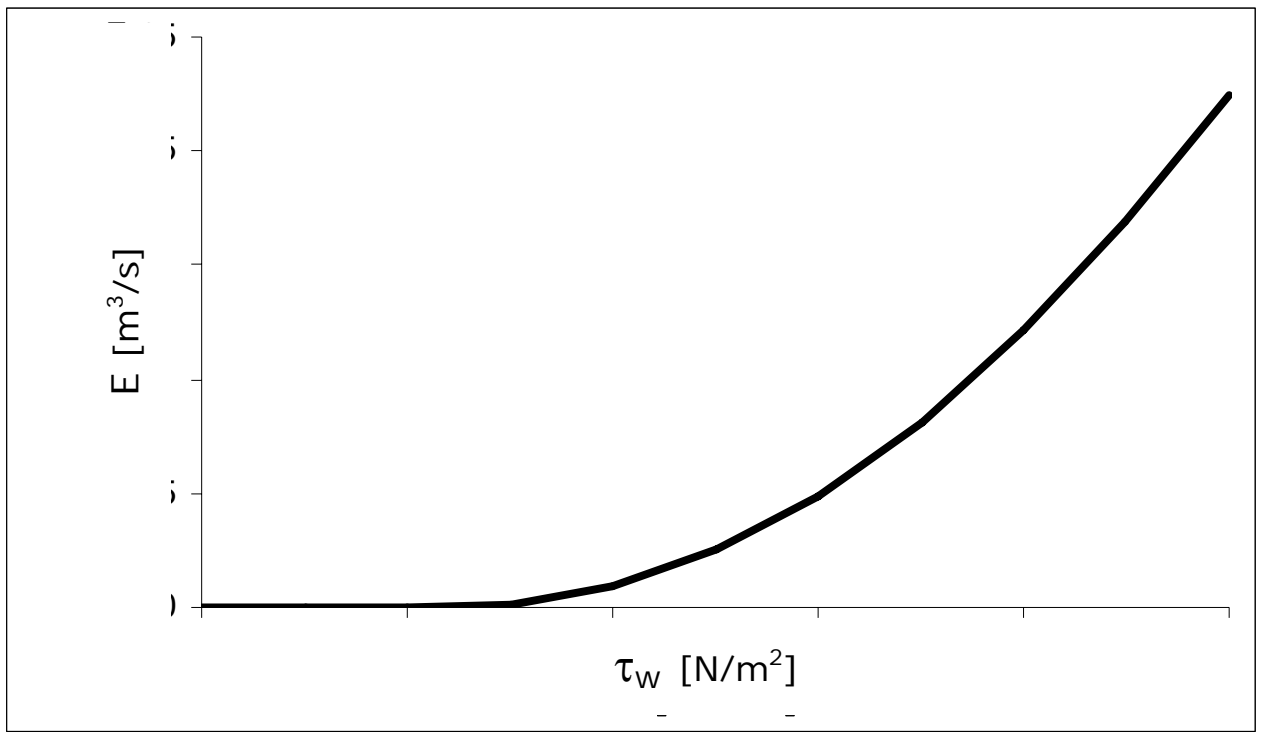

Figure 3.2: Plot of entrainment versus shear stress, Eqn. 3-18.

where $\mu$ is the molecular viscosity, $\mathrm{u}$ is the velocity component tangent to the wall and $\mathrm{n}$ is the direction normal to the wall. However this relation requires the reconstruction of the two-dimensional flow field and this brings extra burden into the solution whereas an equivalent approach may be introduced to eliminate this step. Therefore, the shear stress acting on the mucus lining of the respiratory tract can be written in terms of wall shear stress and could easily be calculated from the one-dimensional flow solution. This approach eliminates the reconstruction of the two-dimensional flow field which speeds up the calculations without any significant loss of the accuracy in the present model.

$$
\tau_{\mathrm{w}}=\frac{\mathrm{C}_{\mathrm{f}}}{2} \rho \mathrm{U}_{\mathrm{avg}}^{2}
$$

Equation 3-20 relates the wall shear stress to the friction, density and average velocity of the flow, which are already available through the onedimensional flow solver. Wall shear stress is calculated using this relation 
with the assumption of zero mucus velocity. In other words, the velocity of the mucus on the wall is very small compared to that of air passing through.

In addition to the calculation of the shear stress, the value of critical shear stress is needed. This requires another empirical relation. The critical shear stress is the force to overcome to rip some portion of the mucus from the wall. It depends on the physical properties of the mucus. Critical shear force may be written in terms of density, viscosity and surface tension, and the thickness of the mucus (Eqn. 3-22). The selection of variables is the basic assumption of the model development (Table 3.2).

$$
\tau_{\mathrm{cr}}=\mathrm{f}\left(\sigma, \delta, \mu, \mu_{\text {air }}\right)
$$

The functional relation between critical shear stress and these variables is written as follows (Eqn. 3-22).

$$
\tau_{\text {cr }}=\mathrm{C}_{\tau} \frac{\mathrm{We}^{\frac{1}{2}}}{\operatorname{Re}}\left(\frac{\sigma}{\delta}\right)\left(\frac{\mu}{\mu_{\mathrm{air}}}\right)
$$

where We and Re are Weber and Reynolds numbers and defined as $\mathrm{Du}^{2} \rho / \sigma$ and $\mathrm{Du} \rho / \mu$, respectively. The other variables used in this relation are given in Table 3.2.

Table 3.2. Variables used in critical shear stress calculation.

\begin{tabular}{clc} 
Variable & \multicolumn{1}{c}{ Definition } & Unit \\
\hline \hline$\delta$ & Film thickness & $\mathrm{m}$ \\
$\sigma$ & Surface tension & $\mathrm{N} / \mathrm{m}$ \\
$\rho$ & Density & $\mathrm{kg} / \mathrm{m}^{3}$ \\
$\mu$ & Viscosity & $\mathrm{kg} / \mathrm{m} \mathrm{s}$ \\
\hline
\end{tabular}




\subsubsection{Droplet Generation Model}

The droplet entrainment model described above calculates the amount of mucus to be stripped of the walls as a bulk material. Droplet generation model (DGM) takes the turbulence into consideration and determines the sizes of the drops formed (Figure 3.3). The maximum diameter of the droplets formed is function of surface tension and the thickness of the mucus, the dissipation rate of the turbulent kinetic energy and turbulent length scale as given by [44];

$$
D_{\text {max }}=\min \left\{C_{B}\left(\frac{\sigma}{\delta}\right)^{\frac{3}{5}} \varepsilon^{-\frac{2}{5}}, \ell_{\text {tur }}\right\}
$$
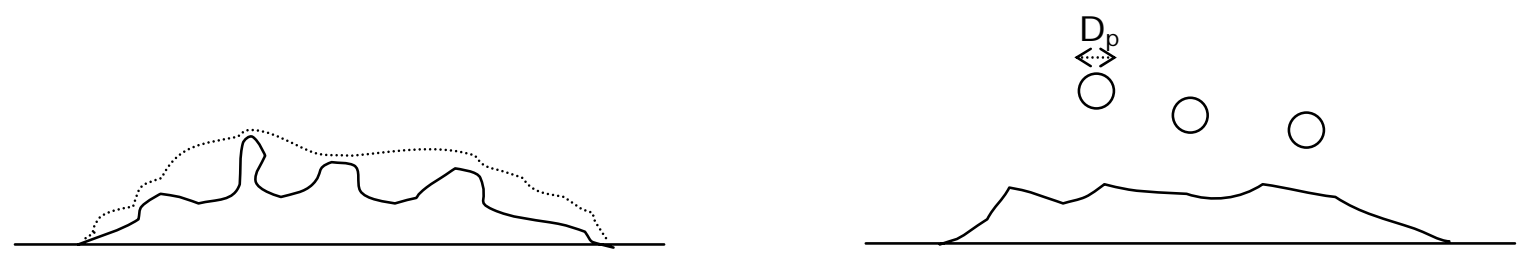

Figure 3.3: Schematic representation of droplet generation model.

If the total amount of mucus entrained, $E$, and the maximum drop size, $D_{\max }$ are known, then the total number of droplets, $n$, can be calculated from the following relation.

$$
\mathrm{n}=\left\lceil\frac{\mathrm{E}}{\frac{4}{3} \pi\left(\frac{\mathrm{D}_{\max }}{2}\right)^{2}}\right\rceil
$$


In this calculation all the droplets are assumed to have the same diameter and the total volume of the drops formed is equal to the total entrainment amount. Since the total number of drops has to be integer, a small modification is needed. One way of resolving this issue is rounding the total number of drops to the next smallest integer. In this case, the maximum particle diameter should be decreased slightly. This is done as follows;

$$
\mathrm{D}_{\max }^{\prime}=2 \sqrt{\frac{\mathrm{E}}{\frac{4}{3} \mathrm{n} \pi}}
$$

The relations 3-24 and 3-25 give the number of droplets to be entrained from the wall and the initial diameter of these droplets, respectively.

\subsubsection{Droplet Breakup Model}

Although the droplet breakup can occur in different ways, it is very common to assume a binary droplet breakup process and has been shown that it agrees well with the experimental measurements [45-47]. During a binary breakup process the mother droplet is divided into two daughter droplets. The process of binary droplet breakup process is shown in Figure 3.4. 


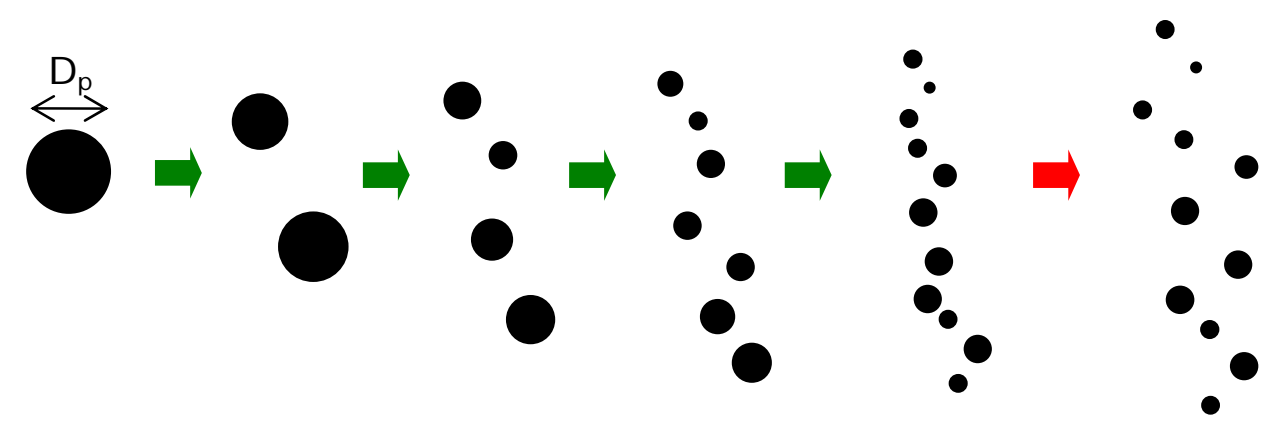

Figure 3.4: Schematic representation of binary droplet breakup model.

The sizes of the daughter droplets are less likely to be the same size. Therefore, the size of one of the daughter droplet is first calculated and the complementary daughter size is then obtained relatively in such a way that the total mass is conserved. The sizes of the daughter droplets cannot be larger than the maximum drop size and smaller than the minimum drop size. The break up process continues until the minimum droplet size is obtained. This is represented in Figure 3.4 with the green arrows. Once the minimum size of droplets is reached, the breakup process stops (red arrow) and the droplets maintain their size until the rest of the simulation. Minimum drop size is a function of physical properties of the fluid making the droplet and the flow parameters and it is defined as:

$$
\mathrm{D}_{\min }=\left(\frac{12 \sigma}{\beta \rho \mathrm{D}}\right)^{3 / 2} \varepsilon^{-1}
$$

where $\varepsilon$ is the turbulent dissipation rate of the air flow, $\sigma$ and $\rho$ are surface tension and density of the mucus fluid, respectively, $D$ is the diameter of the mother droplet, and $\beta$ is an empirical coefficient which is obtained experimentally to be $8.2[31,32]$. 
Knowing the mother droplet diameter and the minimum possible droplet diameter with those mucus properties and at certain flow conditions, the diameter of the first daughter droplet can be calculated. At this time a random function is utilized in order to estimate the diameter of the first droplet. A pseudo-random number is generated within the range of $\left[D_{\min }-D_{\text {mother }}\right]$ and this number is assigned as the diameter of the first daughter droplet. Then the complementary daughter drop size is found as;

$$
D_{\text {complementary }}=\left(D^{3}-D_{\text {min }}^{3}\right)^{1 / 3}
$$

which satisfies the conservation of mass during a break-up process.

\subsubsection{Droplet Breakup Frequency}

Droplet breakup frequency depends on a characteristic length and a characteristic velocity. Obviously, droplet diameter can be selected as the characteristic length. However, the characteristic velocity can be defined as a function of droplet physical properties and the relative velocity, i.e. the difference between droplet velocity and fluid velocity. Calculation of the relative velocity is not trivial therefore a formulation is sought in measurable quantities. In this case the relative velocity is written as a function of the dissipation rate and the droplet diameter by following Kolmogorov's universal theory [31], which leads to the frequency equation shown below:

$$
\mathrm{f}_{\text {breakup }}=\mathrm{K}_{\mathrm{g}} \sqrt{\beta\left(\varepsilon \mathrm{d}_{0}\right)^{2 / 3}-12 \frac{\sigma}{\rho \mathrm{d}_{0}}} / \mathrm{d}_{0}
$$

where $\mathrm{K}_{\mathrm{g}}$ is found to be 0.25 , experimentally, and $\beta=8.2$ [31,32]. The value of $d_{0}$ is equal to the diameter of the mother droplet. New values of $d_{0}$ 
were updated after each binary breakup. In a more general model the relative velocity could be calculated directly from the equations of motion.

Once a droplet is broken-up into daughter droplets, the mother droplet is set as inactive such that it disappears from the system and is ignored in the rest of the calculations. At the time of this break-up, the present simulation time is set as the birth time for the daughter droplets, as well as the life spans for each droplet is assigned by using the inverse of Equation 3-28. Thus each droplet has information about their life span in other words their break-up time. Once this time is attained, each daughter droplet becomes mother droplets and the break-up process continues until the simulation is over or the minimum droplet size is reached by every droplet in the system.

\subsubsection{Turbulent Kinetic Energy Equation}

Integration of the aerosol generation and the entrainment model into the one-dimensional flow field requires information about dissipation rate and turbulent kinetic energy from the main flow solver. This information is passed to the model through the solution of a one-dimensional integral turbulent kinetic energy equation.

Essentially, the solution of turbulent kinetic energy equation and passing the results to the model calculations is the most important interaction between the one-dimensional flow solver and the droplet models.

The derivation of the integral turbulent kinetic energy equation can be found elsewhere [33]. Here, the final form of the integral turbulent kinetic energy equation is shown (Eqn. 3-29). 


$$
\frac{\partial}{\partial t}(\rho A \Delta x k)=\left(\rho u A k-\Gamma_{k} \rho A \frac{\partial k}{\partial x}\right)_{w}-\left(\rho u A k-\Gamma_{k} \rho A \frac{\partial k}{\partial x}\right)_{e}+G-D
$$

where $\mathrm{k}$ represents the turbulent kinetic energy, $\rho$ and $\mathrm{u}$ are density and average flow velocity, respectively, $A$ is the cross-sectional area normal to the flow direction and $\Gamma_{k}$ is the effective viscosity defined as:

$$
\Gamma_{k}=v+\frac{v_{t}}{\sigma_{\text {tur }}}
$$

here $v$ is the molecular viscosity and ${ }^{V_{t}}$ is turbulent eddy viscosity and $\sigma_{t u r}$ is a kind turbulence Prandtl-Schmidt numbers which is taken as 1 in this study. Turbulent viscosity is calculated from:

$$
v_{t}=C_{v} u \ell_{t}
$$

where $C_{v}$ is the model coefficient and $\ell_{t}$ is a turbulent length scale, which is taken to be proportional to the boundary layer thickness. Model coefficient is $C_{r}$ set equal to 0.5 and the turbulent length scale is taken as 0.1 of the radius.

$G$ and $D$ denote turbulent kinetic energy generation and dissipation, respectively, and they are calculated from:

$$
\begin{aligned}
& G=\tau_{w} u A_{\text {lateral }}+\mu_{t} \frac{\partial u}{\partial x} A u \\
& D=C_{D} \frac{k^{3 / 2}}{\ell_{t}} \rho A \Delta x
\end{aligned}
$$


where $C_{D}$ is a model coefficient and taken as 2 and $\ell_{t}$ is the turbulent length scale and taken as 0.1 of the radius. It should be noted that the values cited above for the model coefficients are not selected arbitrarily. This selection process was based on the past literature on turbulent flow models and some arbitration performed during this study.

Wall shear stress in generation term is defined as:

$$
\tau_{w}=\mu_{t} \frac{\partial u}{\partial r} \cong \frac{C_{f} \rho}{2} u_{a v g}^{2}
$$

The approach outlined above eliminates the need for the reconstruction of the two-dimensional flow field. This, in turn, improves the efficiency of the solver by decreasing the computational time in addition to the errors arising in the reconstruction process. The one-dimensional solver is based on the lumped model and it solves the integral one-dimensional continuity and momentum equations. Solution of the turbulent kinetic energy equation based on this lumped model is more proper than indirectly obtaining the information through the reconstructed flow field, which may include other inaccuracies. 


\section{CHAPTER 4: \\ APPLICATI ONS}

"I am a great believer in luck, and I find the harder I work the more I have of it." T. Jefferson

\subsection{Flow Field Calculations}

The pseudo two-dimensional larynx flow (PTL) model has been verified and compared with the FLUENT results for two cases; a laminar case and a low Reynolds number turbulent case. Then, the PTL model was applied to the actual geometry in a human respiratory system. The Reynolds number based on the maximum average velocity and glottal opening, are calculated as 1200,3000 , and 15000 for the laminar, low Reynolds number turbulent case, and the breathing case, respectively.

The methodology of a pressure-correction algorithm, in conjunction with a projection method, has been employed for obtaining the average velocity within upper airways. It has already been shown that this approach presented promising results for a one-dimensional flow problem [36]. This approach has initially been applied to a steady converging-diverging nozzle flow and the results have been compared with the analytical solution [1]. It was shown that the solution was reasonably accurate. In the present study, the validated code for steady flow has first been applied to the same converging diverging nozzle with oscillatory boundary conditions. Then it has been applied to the larynx geometry with a pressure boundary condition 
$(\mathrm{P}=1 \mathrm{~atm})$ at the inlet and a sinusoidal boundary condition at the outlet $[2,7]$. The results obtained with these boundary conditions are compared with the two-dimensional flow field results obtained for the same conditions using the FLUENT CFD software package [7]. The results lead to the application of more realistic boundary conditions relevant to a human respiratory cycle. These boundary conditions are oscillatory but different than the simple sinusoidal boundary conditions.

The geometry used in this study (Fig.4.1) is a simplified version of the actual larynx, with the axisymmetric assumption. The dimensions and the ratios are obtained from several references, and the figures obtained from these references have been digitized by the known dimensions of certain locations. The dimensions used in the simulations are for an average male human larynx with an average glottal opening.

The PTL model calculations are carried out on a uniform staggered grid. The grid points in the axial and radial directions are 41 and 21, respectively. For the FLUENT case, an unstructured grid with 2746 cells was used.

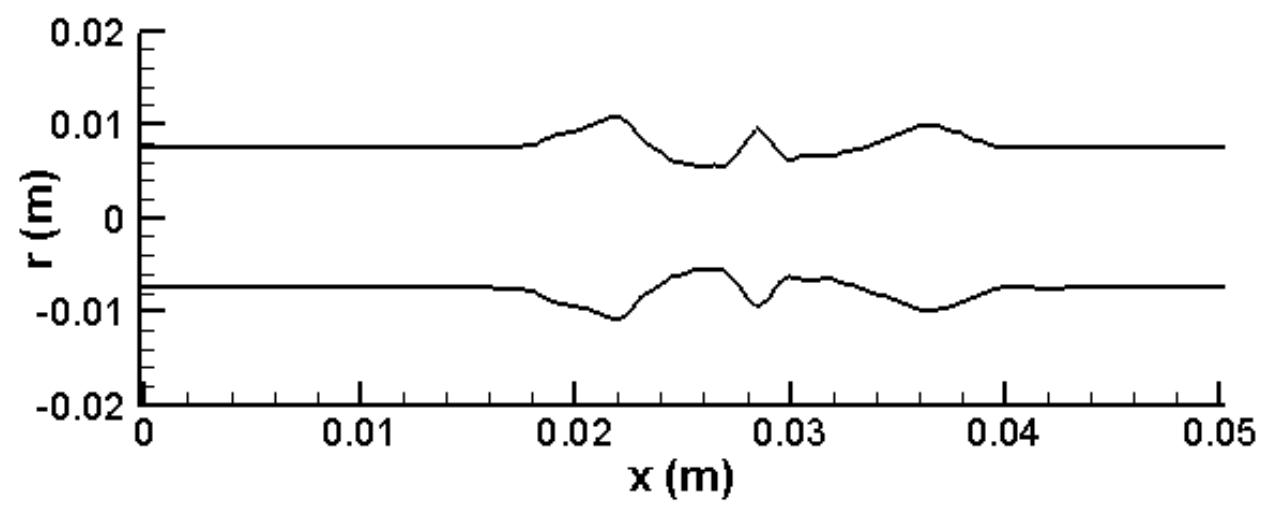

Figure 4.1: Geometry of the system used in the simulations. 


\subsubsection{Laminar Flow Case}

In this case, at the inlet of the larynx (supraglottic area), which is the mouth side, atmospheric pressure is applied. At the exit (subglottic area), which is the lung side, pressure is oscillated around the atmospheric pressure as seen in Figure 4.2 .

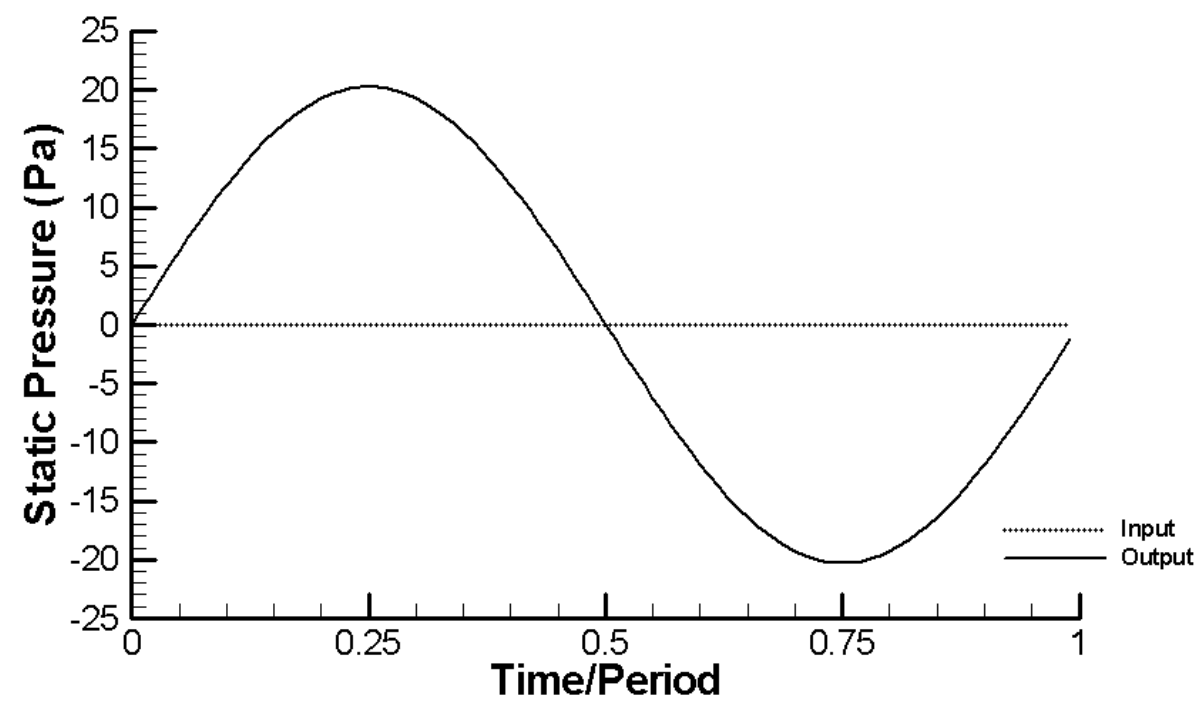

Figure 4.2: Sinusoidal pressure variation at the boundaries.

Comparing the mass flowrate of both methods is the first constraint. Mass flowrate variation in one complete cycle has shown that the present model agrees quiet well with the FLUENT results with a maximum of $10 \%$ difference (Fig. 4.3). The axial velocity and pressure profiles along the axial direction are compared in Figures 4.4 and 4.5. These figures show a very good agreement with the FLUENT results.

The two-dimensional velocity profiles were also compared to check the performance of the PTL model. For this comparison, the velocity profiles were recorded at several axial locations and plotted in Figures 4.6 - 4.8. The velocity profiles obtained by the PTL model also show a good agreement with the FLUENT results at points B and C (Fig. 4.6,4.7), however, at point D the 
same level of agreement could not be achieved (Fig. 4.8). See Fig. 4.1 for relative locations of the axial points $B, C$, and $D$.

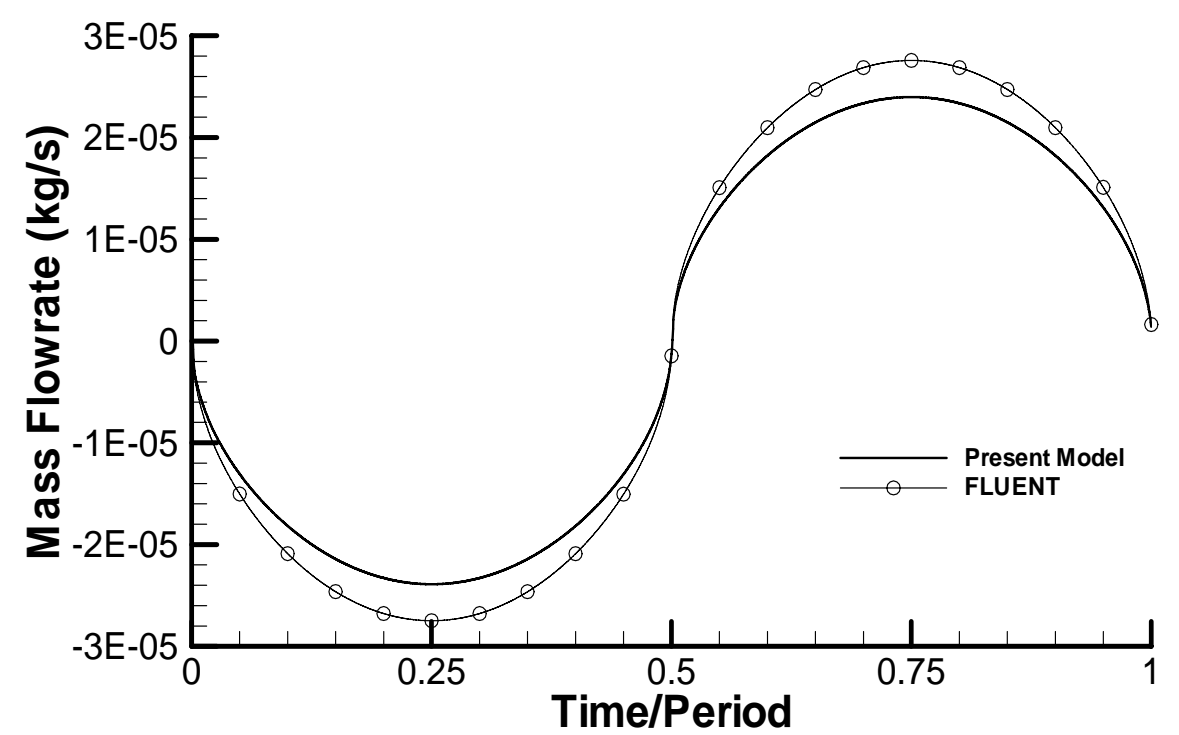

Figure 4.3: Mass flowrate variation in one complete cycle for laminar case.

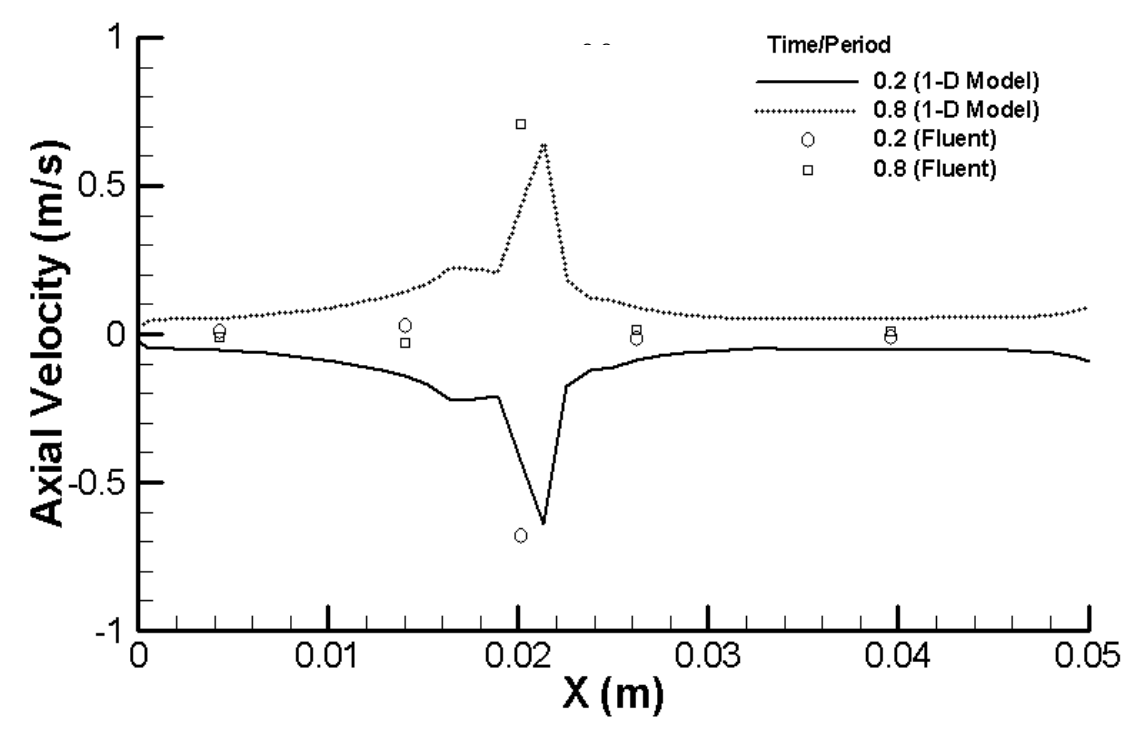

Figure 4.4:. Axial velocity variation at centerline with time along axial direction for laminar case. 

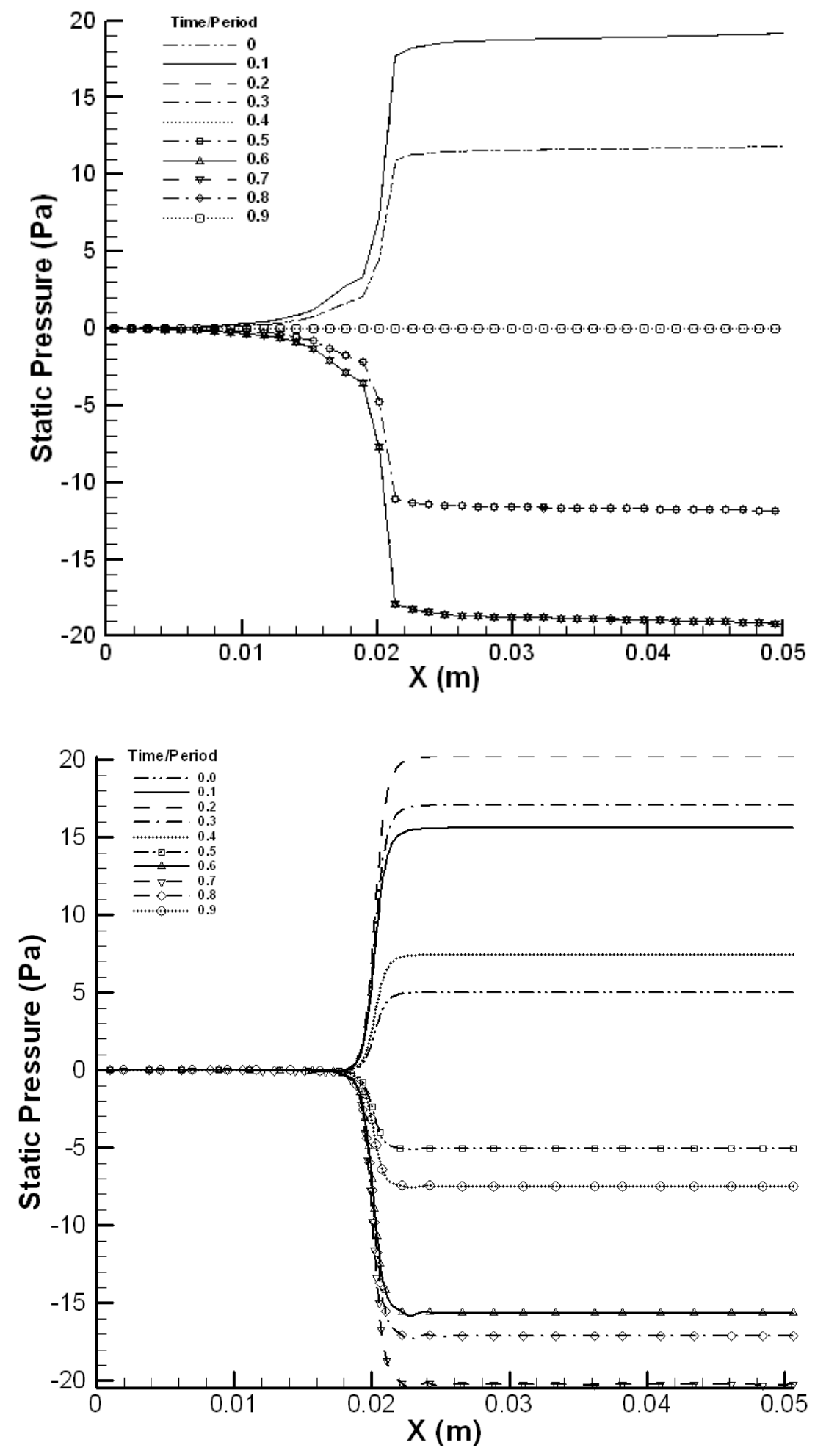

Figure 4.5: Pressure variation with time along axial direction for laminar case by (a) present model and (b) FLUENT. 

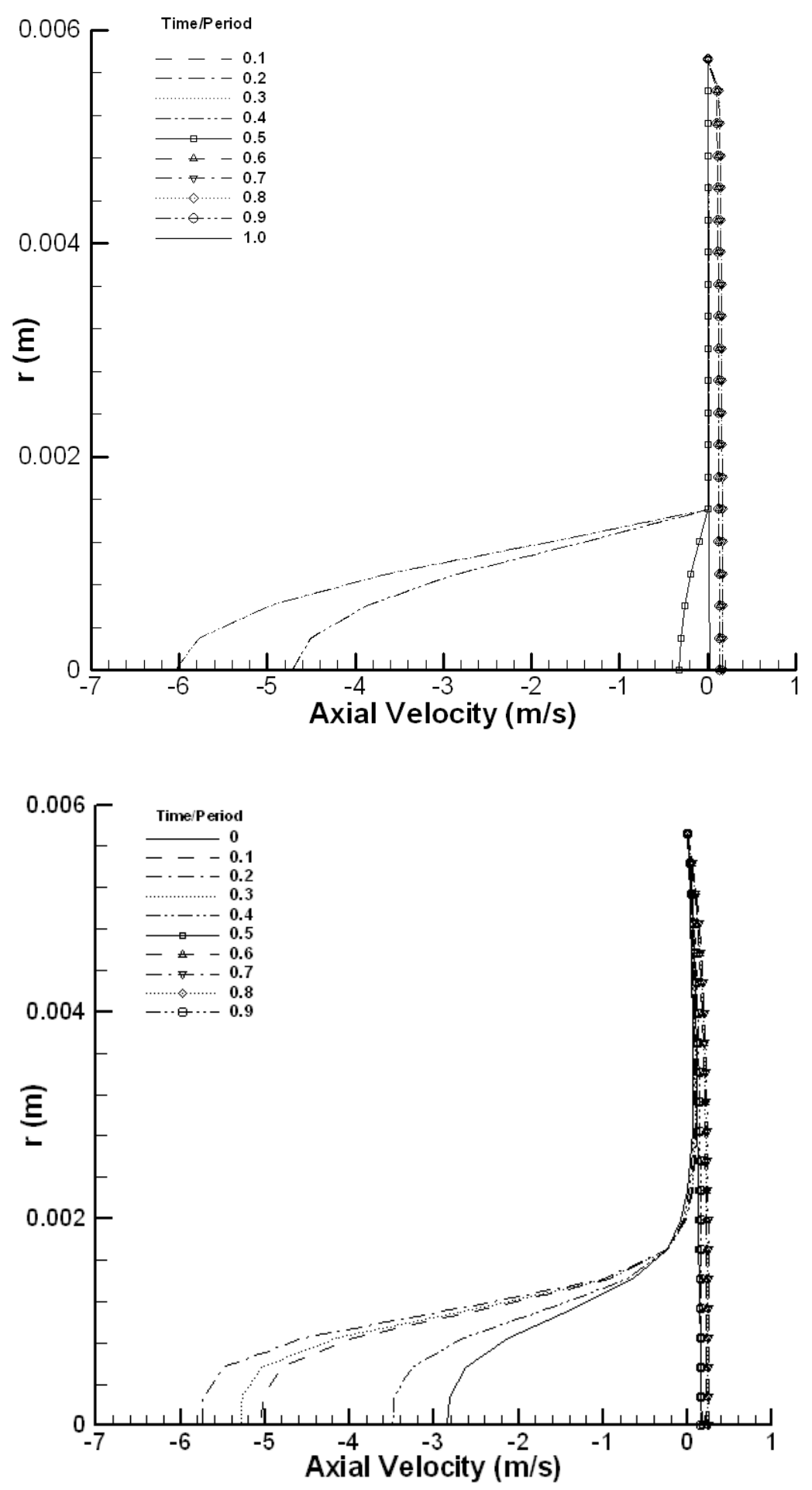

Figure 4.6: Velocity profiles at point B for laminar case by (a) present model and (b) FLUENT. 

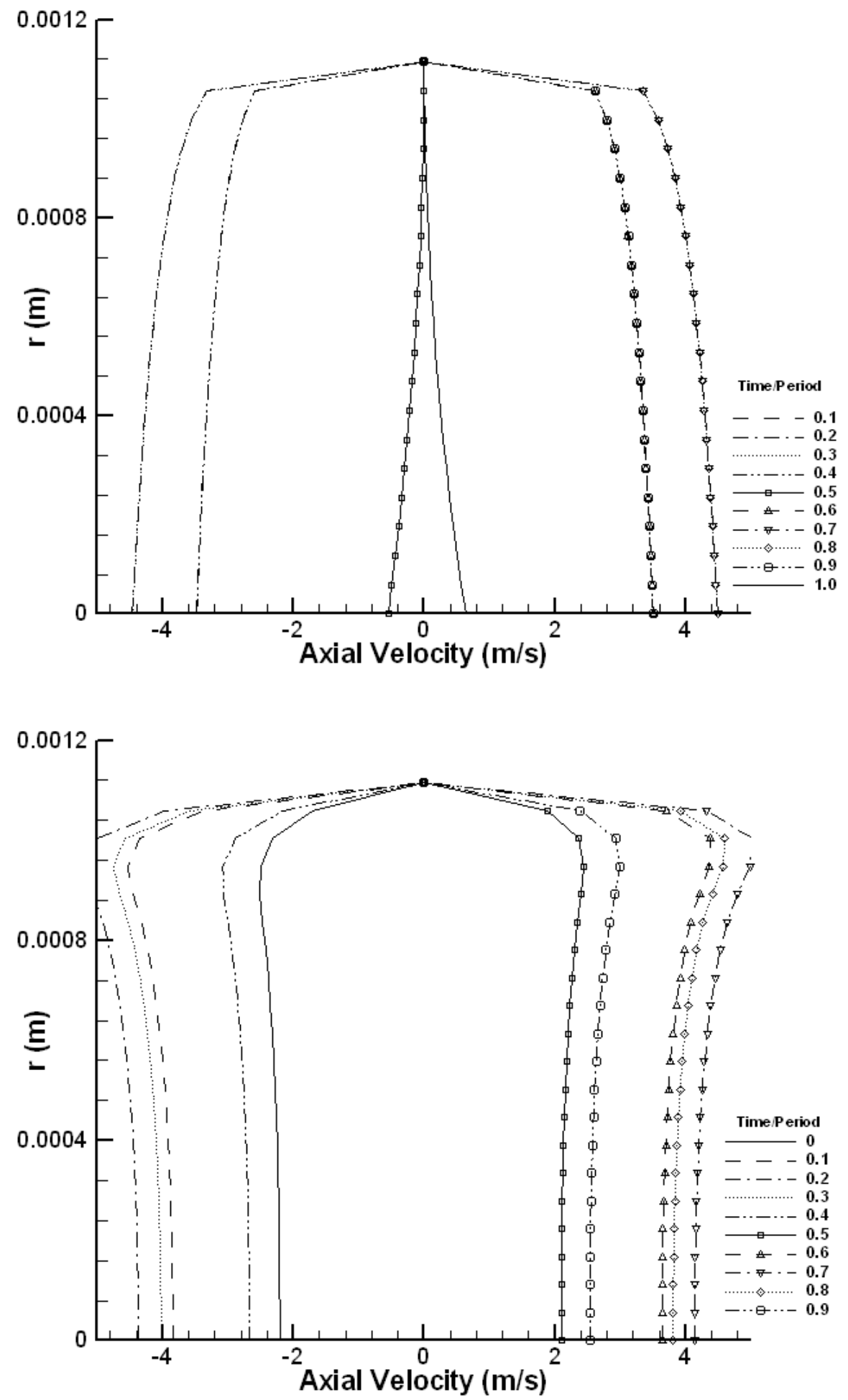

Figure 4.7: Velocity profiles at point C for laminar case by (a) present model and (b) FLUENT. 

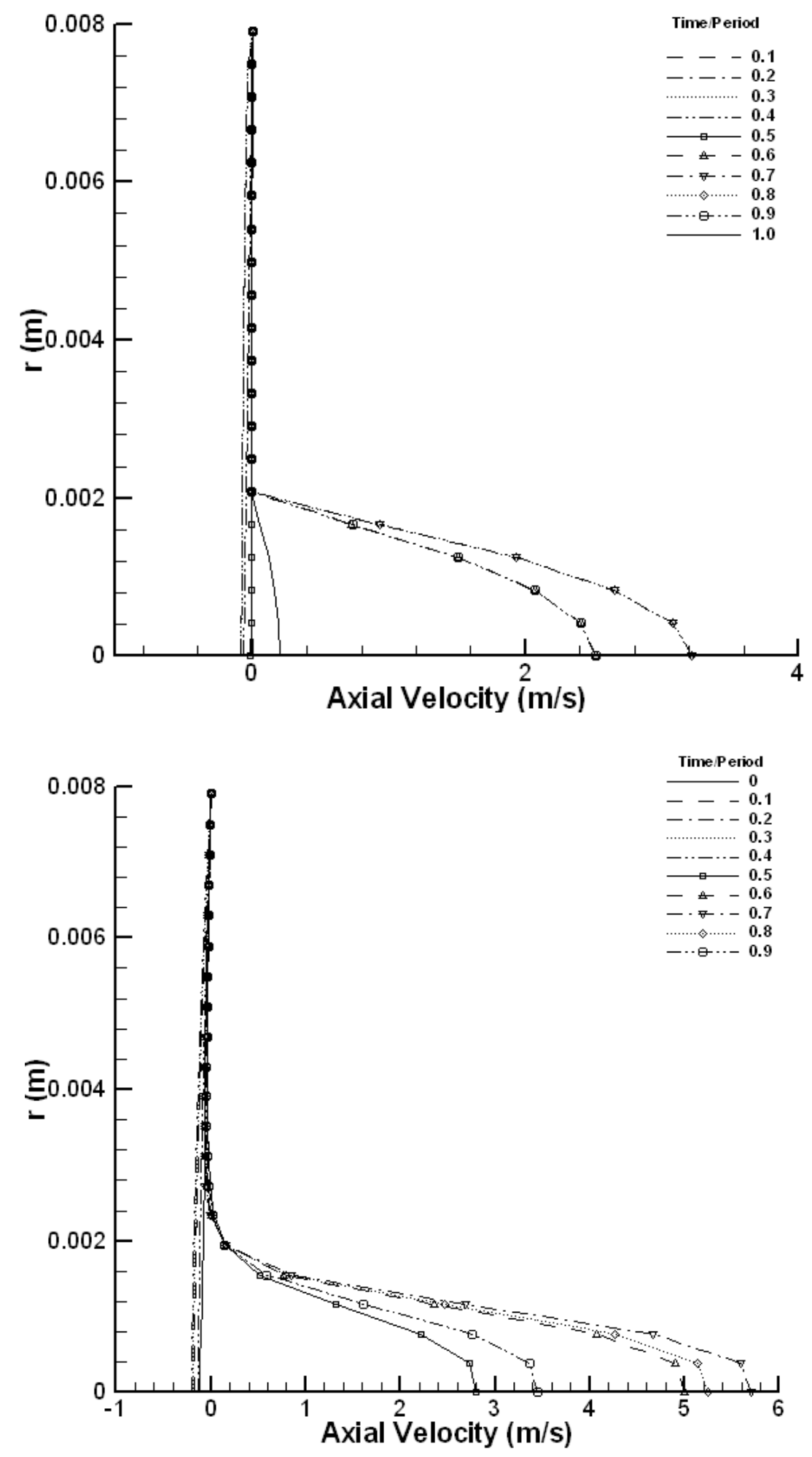

Figure 4.8: Velocity profiles at point $D$ for laminar case by (a) present model and (b) FLUENT. 


\subsubsection{Low Reynolds Number Turbulent Flow Case}

The mass flowrate from the present model and that of the FLUENT calculations also agrees well for low Reynolds number turbulent flow case. In FLUENT calculations the renormalization Group Theory (RNG) $\mathrm{k}-\varepsilon$ model with low Reynolds number correction was employed. (Fig. 4.9).

The pressure profiles along the axial direction depicted in 4.10 also show a very good agreement between the present model and the FLUENT results. As it is seen from this figure, the sharp pressure gradient in the axial direction at various time steps can be captured very well.

The velocity profiles obtained by the PTL model for the low Reynolds number turbulent flow case show similar agreement with the FLUENT calculations as in the laminar case (Figs. 4.11 - 4.13).

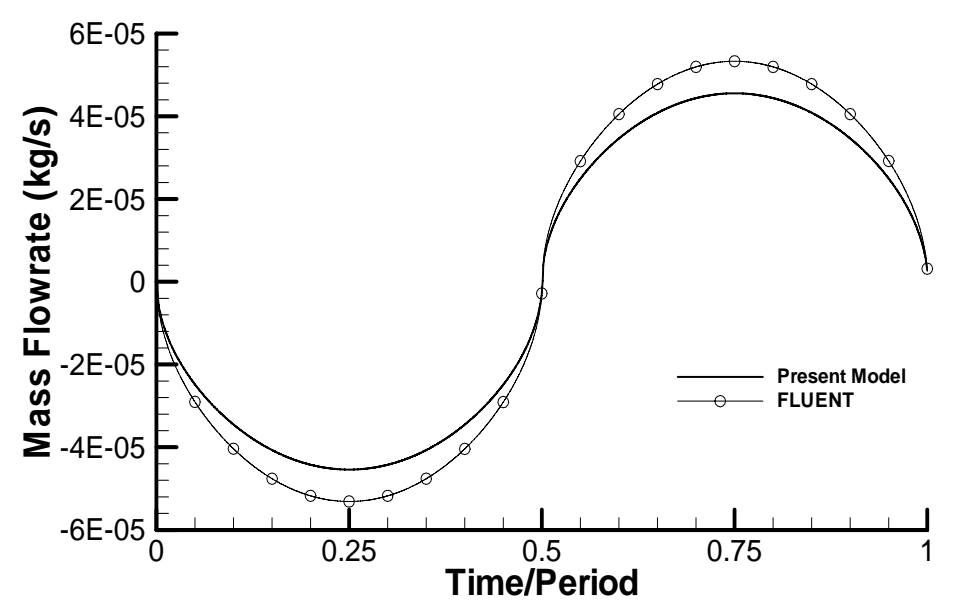

Figure 4.9: Mass flow rate variation in one complete cycle for low Reynolds number turbulent flow case. 

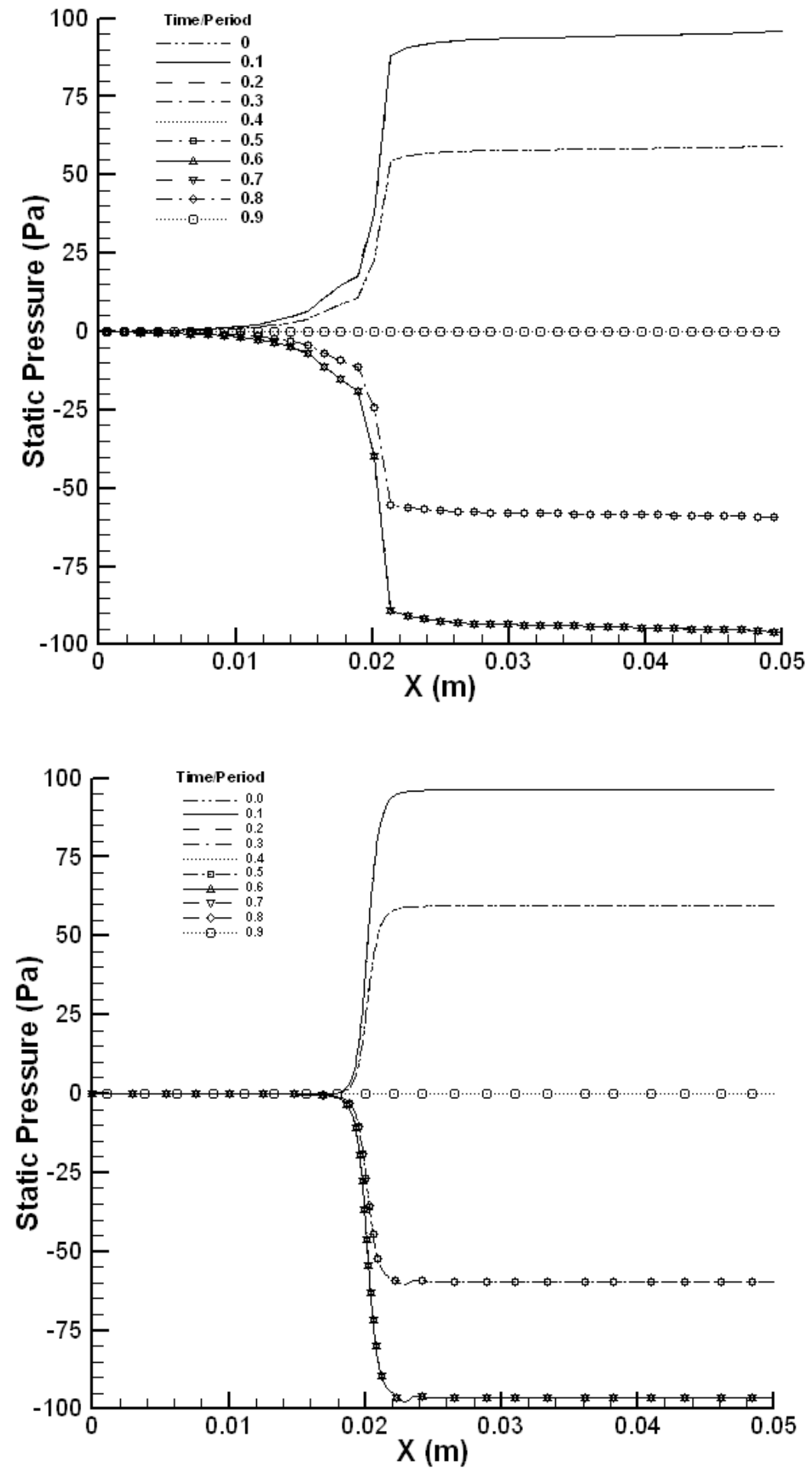

Figure 4.10: Pressure variation with time along axial direction for low Reynolds number turbulent case by (a) present model and (b)

\section{FLUENT.}



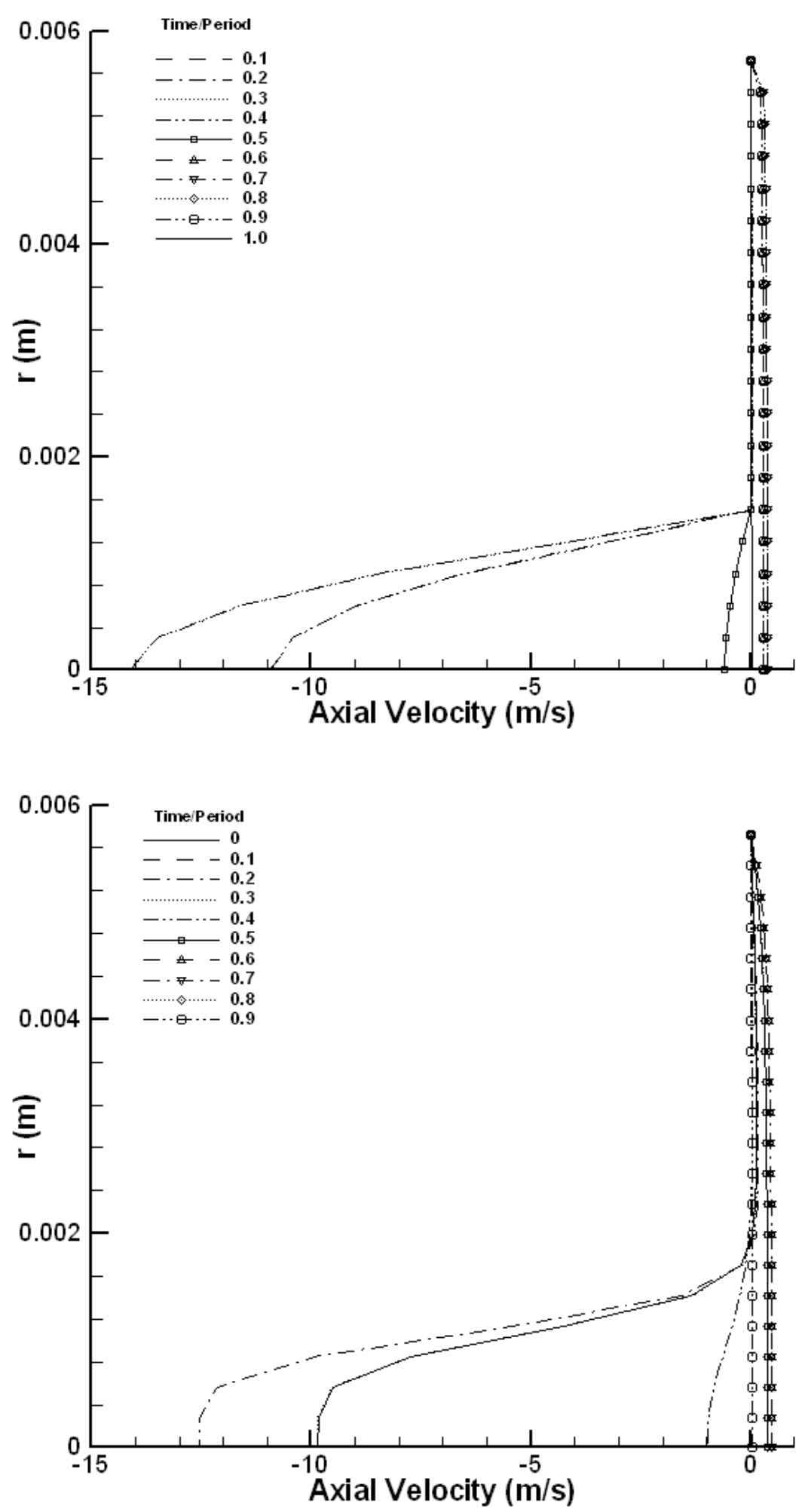

Figure 4.11: Velocity profiles at point B for low Reynolds number turbulent case by (a) present model and (b) FLUENT. 

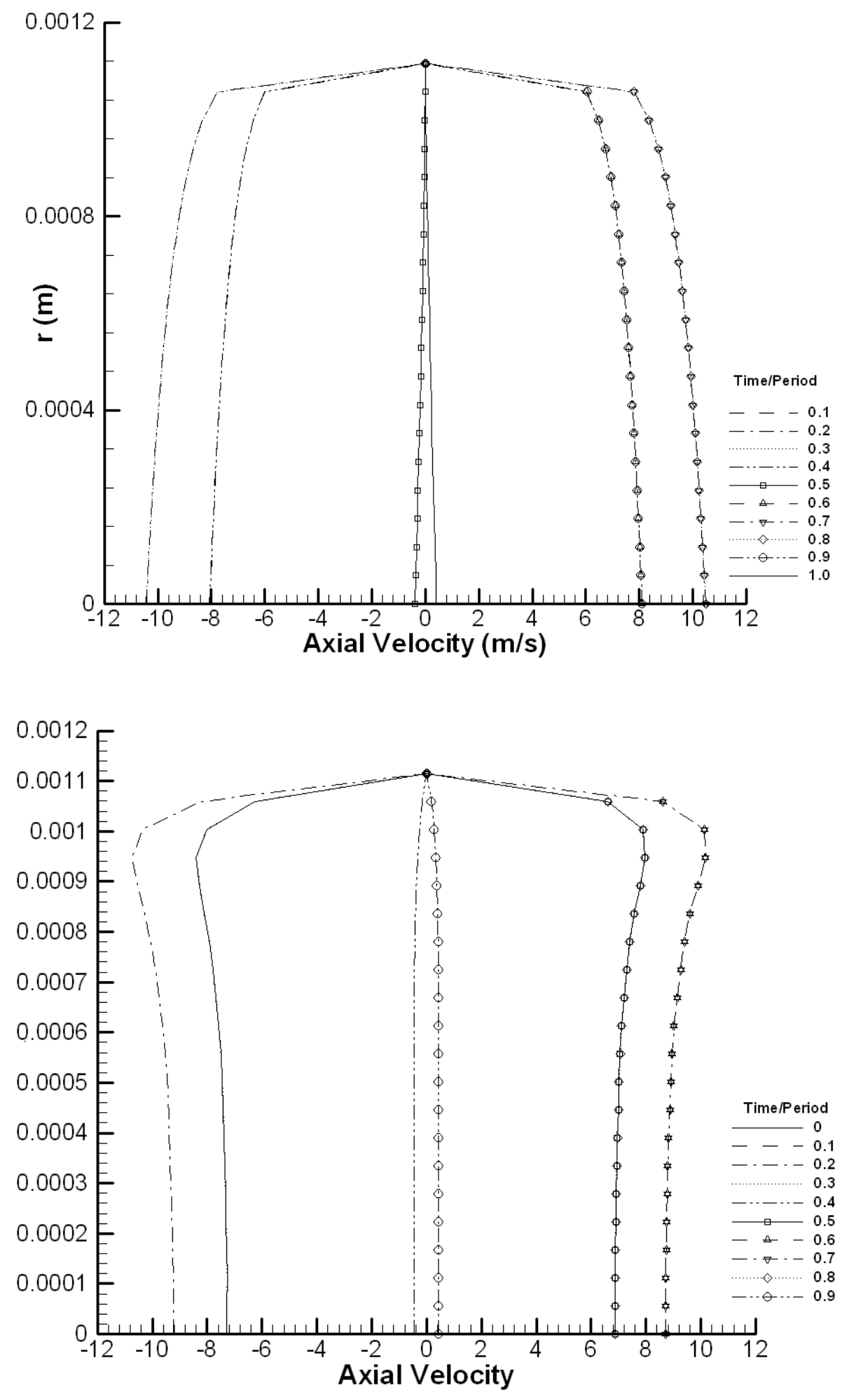

Figure 4.12: Velocity profiles at point $C$ for low Reynolds number turbulent case by (a) present model and (b) FLUENT. 

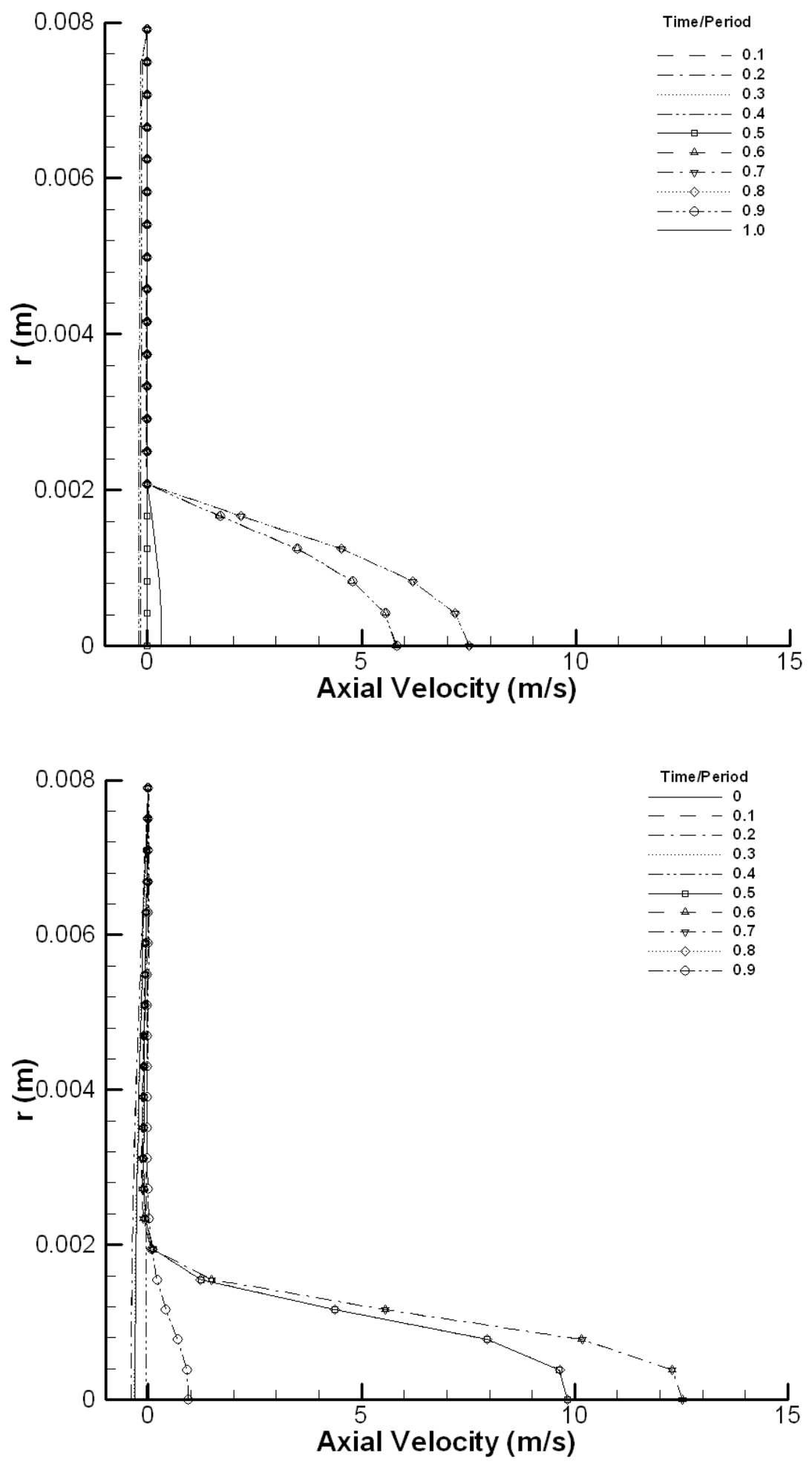

Figure 4.13: Velocity profiles at point $D$ for low Reynolds number turbulent case by (a) present model and (b) FLUENT. 


\subsection{Respiratory Cycle}

For this case, the trend of the pressure variation with time has been obtained from the medical literature [13]. As can be seen from Figure 4.14, the realistic pressure profile significantly differs from a sinusoidal wave. Although it changes between a minimum and a maximum, the change is not symmetric, due to the nature of the respiratory cycle. It should also be noted that there are some moments where the pressure at both ends are equal which is called the resting period. This pressure cycle is applied to generate air flow to closely represent the flow physics in the larynx.

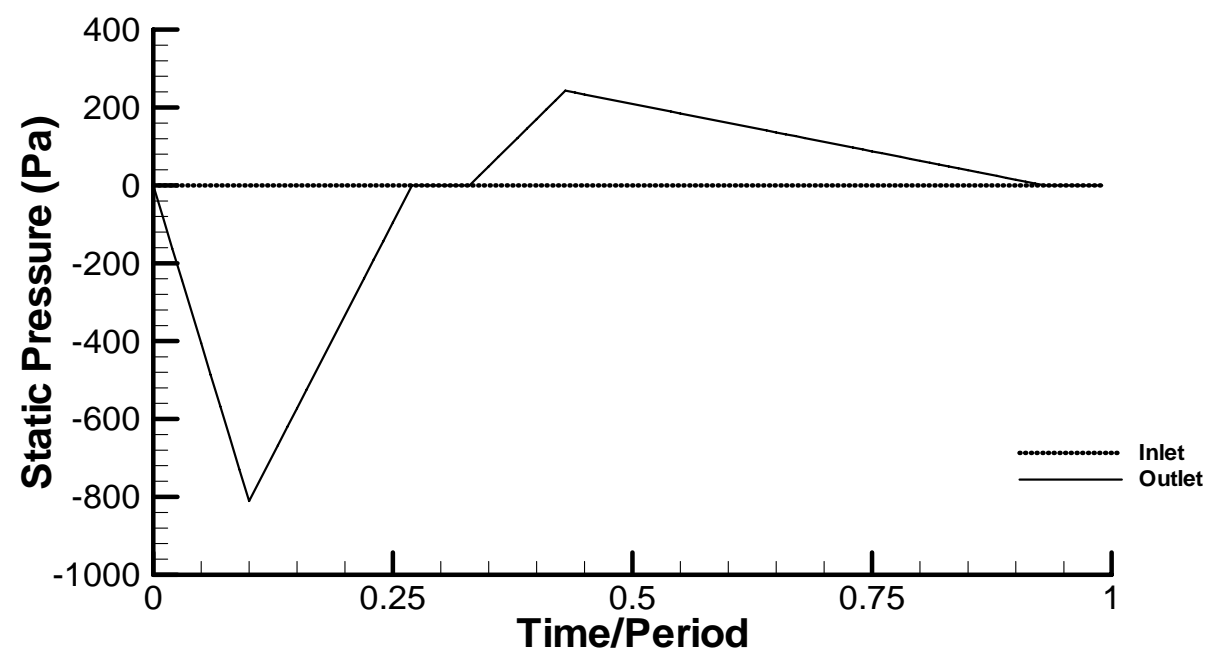

Figure 4.14: Realistic pressure variation at the boundaries.

Although, the main goal was to simulate the actual respiratory cycle, using a pressure drop led to too high velocities at the glottal opening. Therefore, the same boundary condition function has been applied with a lower pressure drop in order to obtain acceptable velocities at the glottal opening. Figure 4.15 shows the mass flowrate variation for one complete cycle. Here, the total inhalation and exhalation air volumes are compared to check conservation of mass in the system. 
The axial velocity profiles along the axial direction at different time steps are plotted in Fig. 4.16. The velocity profiles along axial direction shows the same trend as the other two cases with a higher maximum velocity.

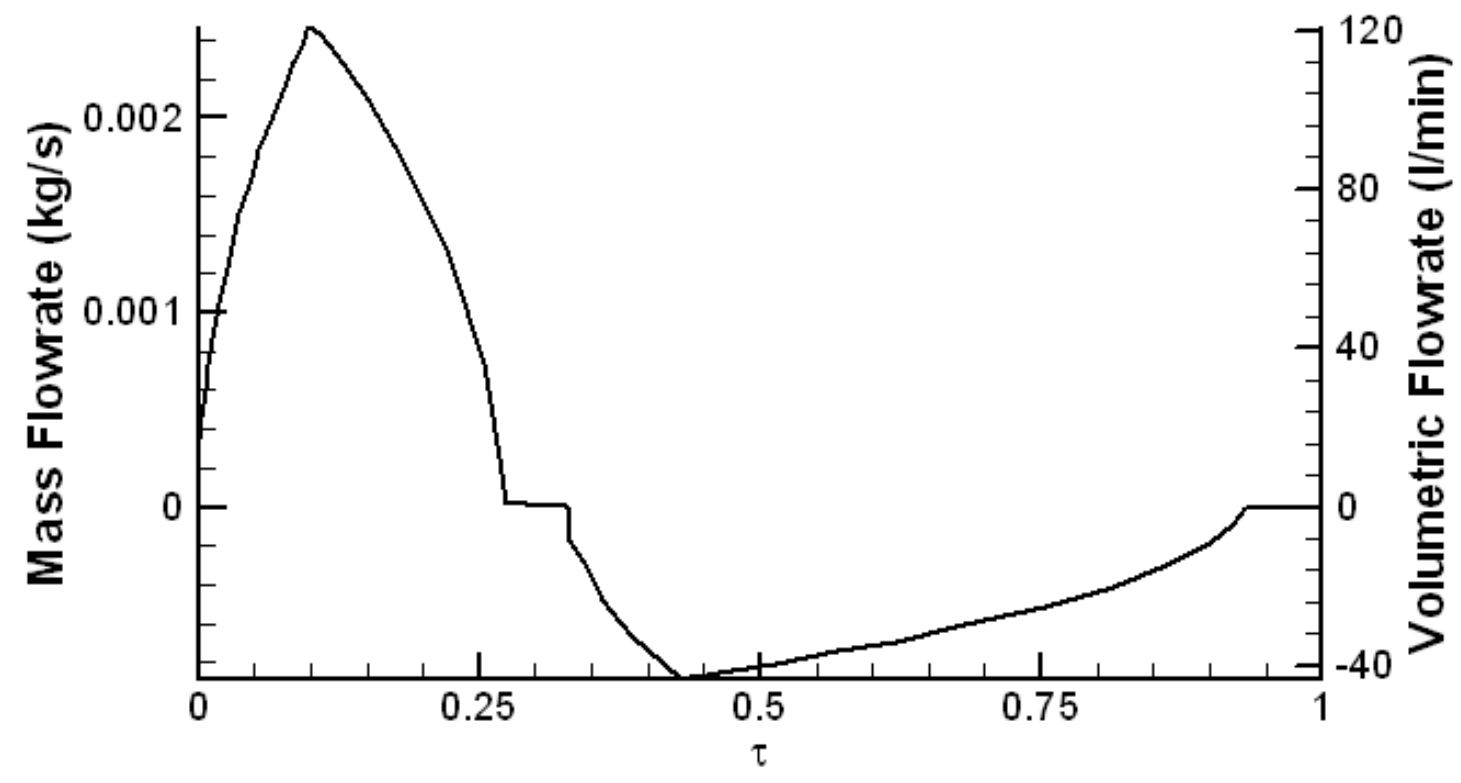

Figure 4.15: Mass flow rate variation in one complete cycle for breathing case.

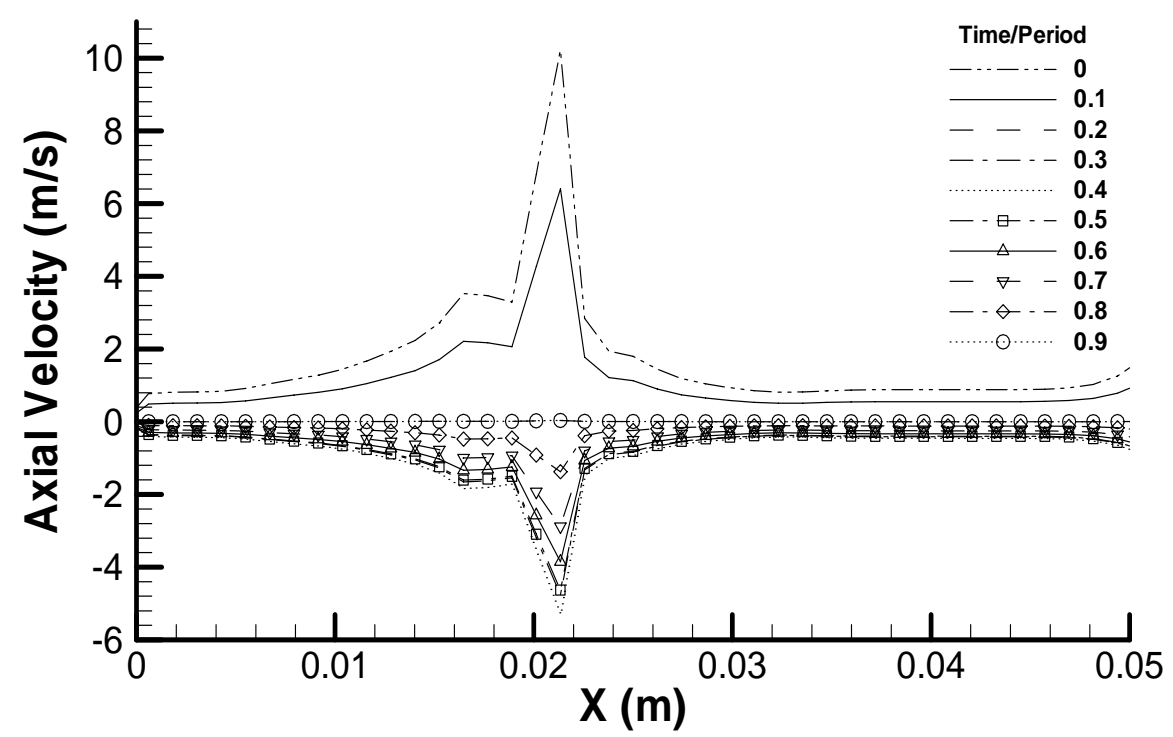

Figure 4.16: Axial velocity variation at centerline with time along axial direction for breathing case. 


\subsection{Cough Simulations}

\subsubsection{One-Dimensional Flow Analysis}

After simulating the breathing conditions with PTL model, the next step has been the simulation of the coughing conditions. In this study only one dimensional analysis is conducted on the improved geometry based on threedimensional scan data obtained from NIOSH [49]. The reconstruction of the two-dimensional flow field during the cough is left for a future study.

In the cough simulations the required boundary condition is obtained from the data provided by NIOSH [3]. The researchers at NIOSH conducted some experiments on coughing [4,5]. Five people have been used and each person coughed three times ending up in fifteen different coughing conditions. The data is available in flowrate versus time format as can be seen in Figure 4.17.

It is seen from Figure 4.17 all the flowrate curves show a similar trend with some minor differences. There is a peak in the flowrate at the early stages of the coughing than it decreases and finally reaches approximately to zero. One of the coughing conditions has been selected for the simulations as shown in Figure 4.18.

The extracted data on flowrate has been used to calculate the pressure boundary condition at the lung side. The relation between pressure drop and the flowrate is postulated as;

$$
\Delta \mathrm{P} \propto \dot{\mathrm{m}}^{2}
$$




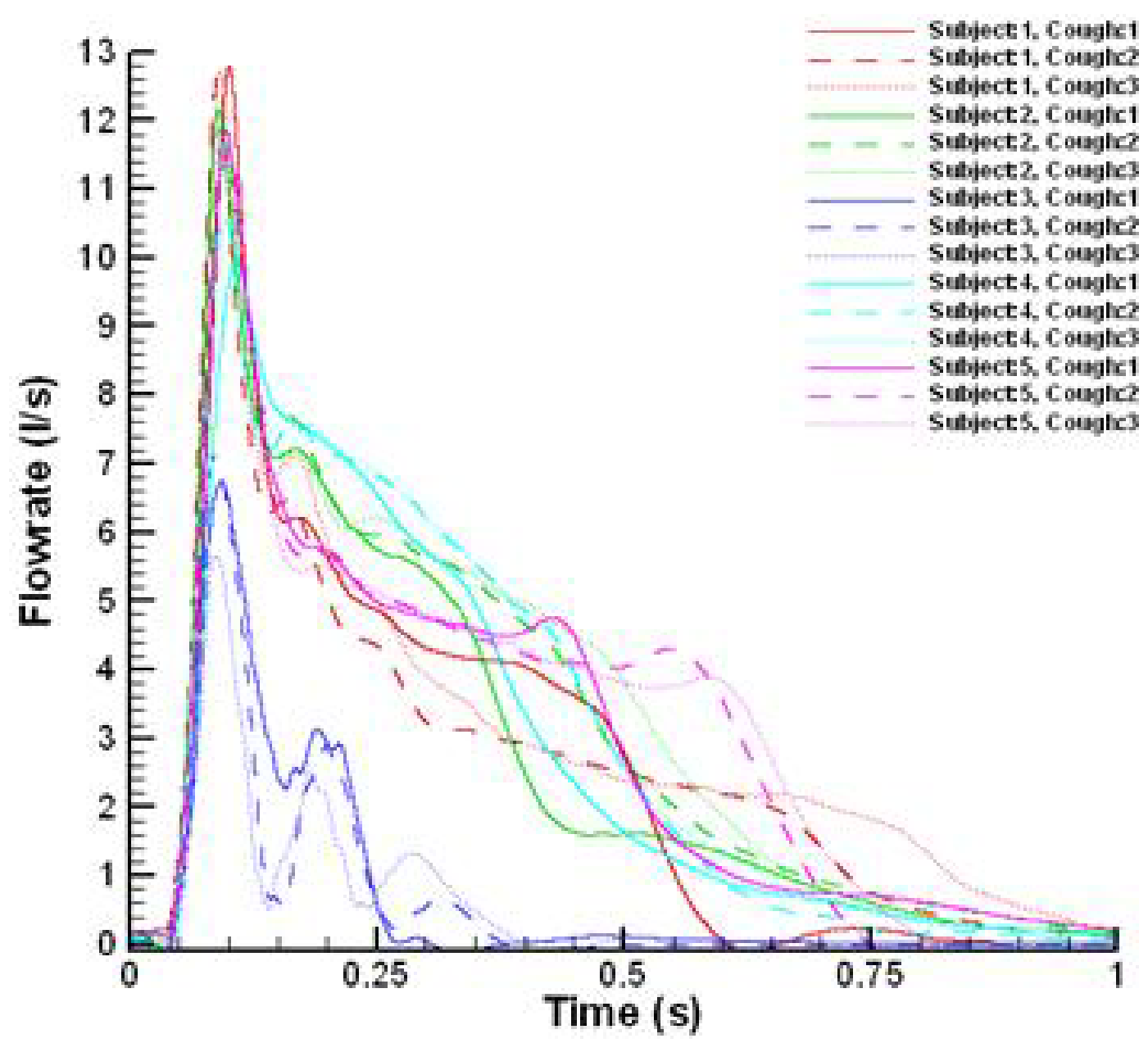

Figure 4.17: Coughing data obtained from NIOSH [5].

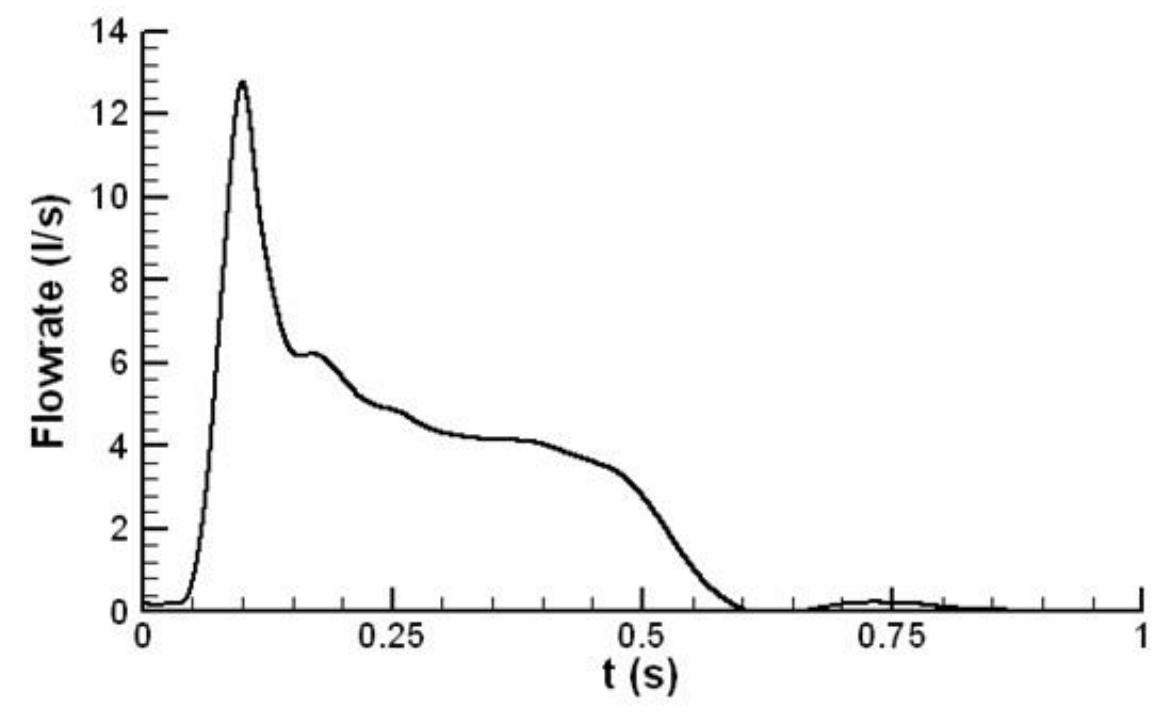

Figure 4.18: Extracted coughing data obtained from NIOSH. 
By using the relation above (Eqn. 4-1) the following pressure trend is obtained as the boundary condition (Fig. 4.19).

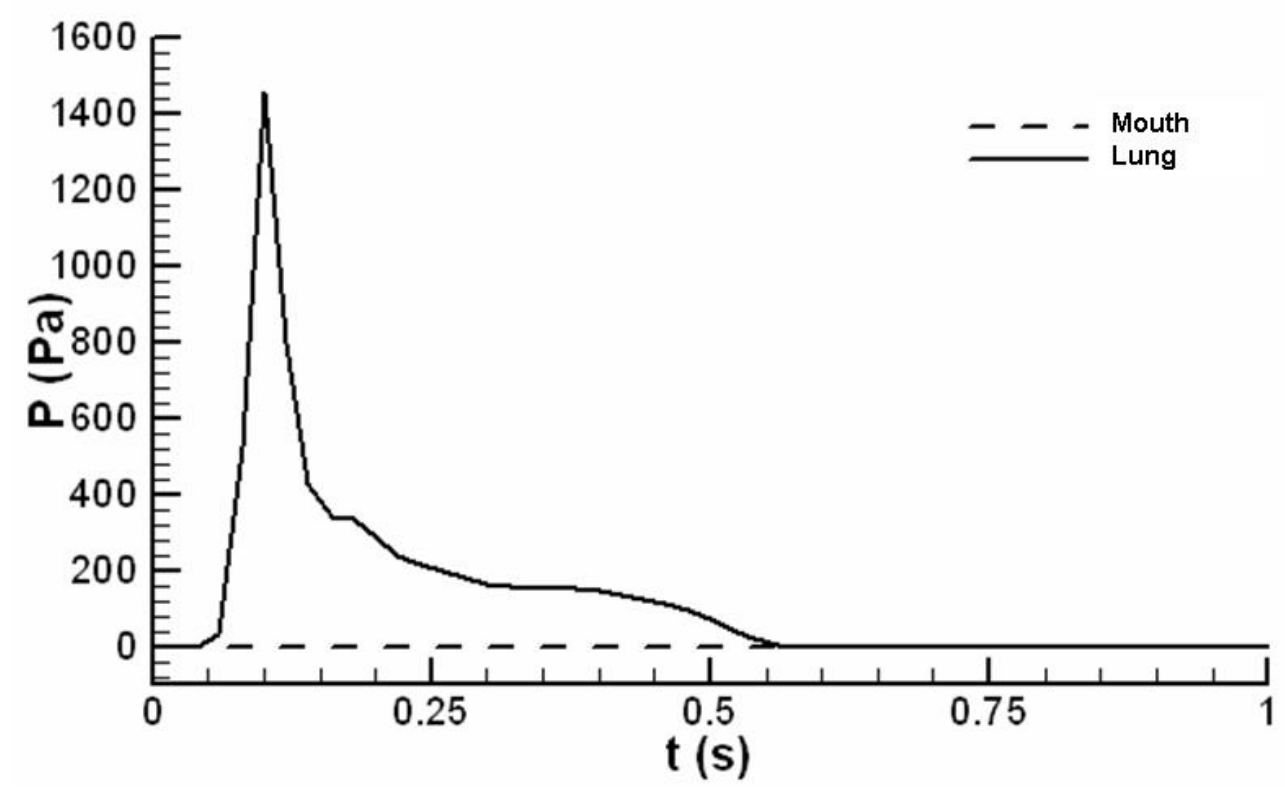

Figure 4.19: Pressure boundary conditions for cough simulations.

Using the pressure variation shown in Figure 4.19, the simulations have been carried out and the following results are obtained. In Figure 4.20, the variation of the effective radius is seen along the axial direction. The effective radius is defined as the equivalent radius of a circular cross section having the same area of the actual cross section at that axial location.

In Figure 4.21 the variation of pressure can be seen at the peak of the cough. There is a significant pressure gradient in the axial direction. This behavior can also be seen in the FLUENT simulations of the same case (Section 4.3.2). Figure 4.22 shows the variation of the axial mean velocity along the axial direction. As expected, the velocity reaches its maximum value at the glottal opening. 


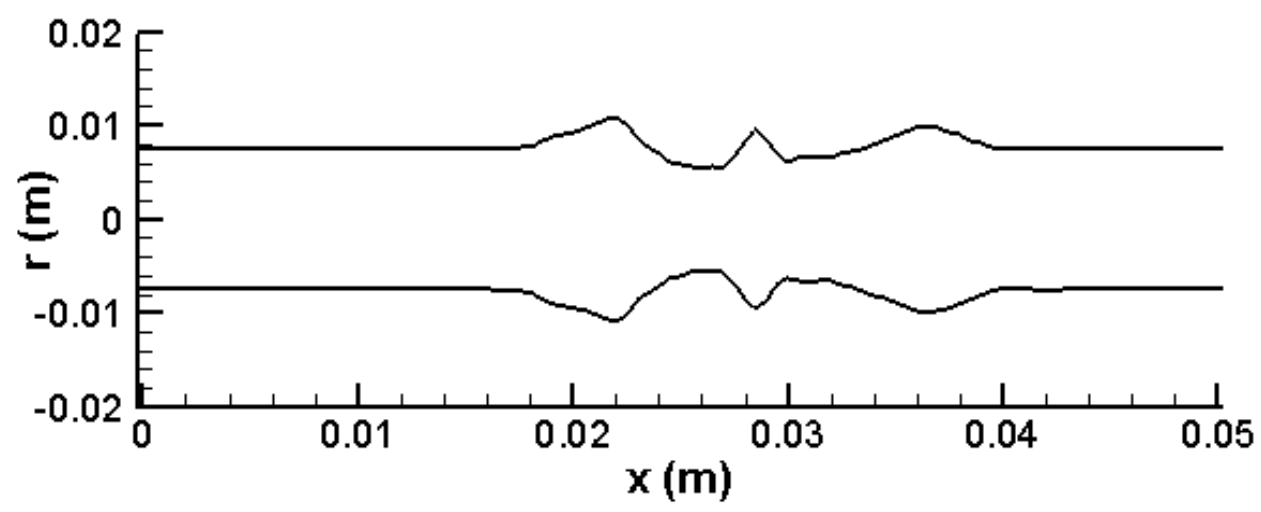

Figure 4.20: Radius versus axial direction.

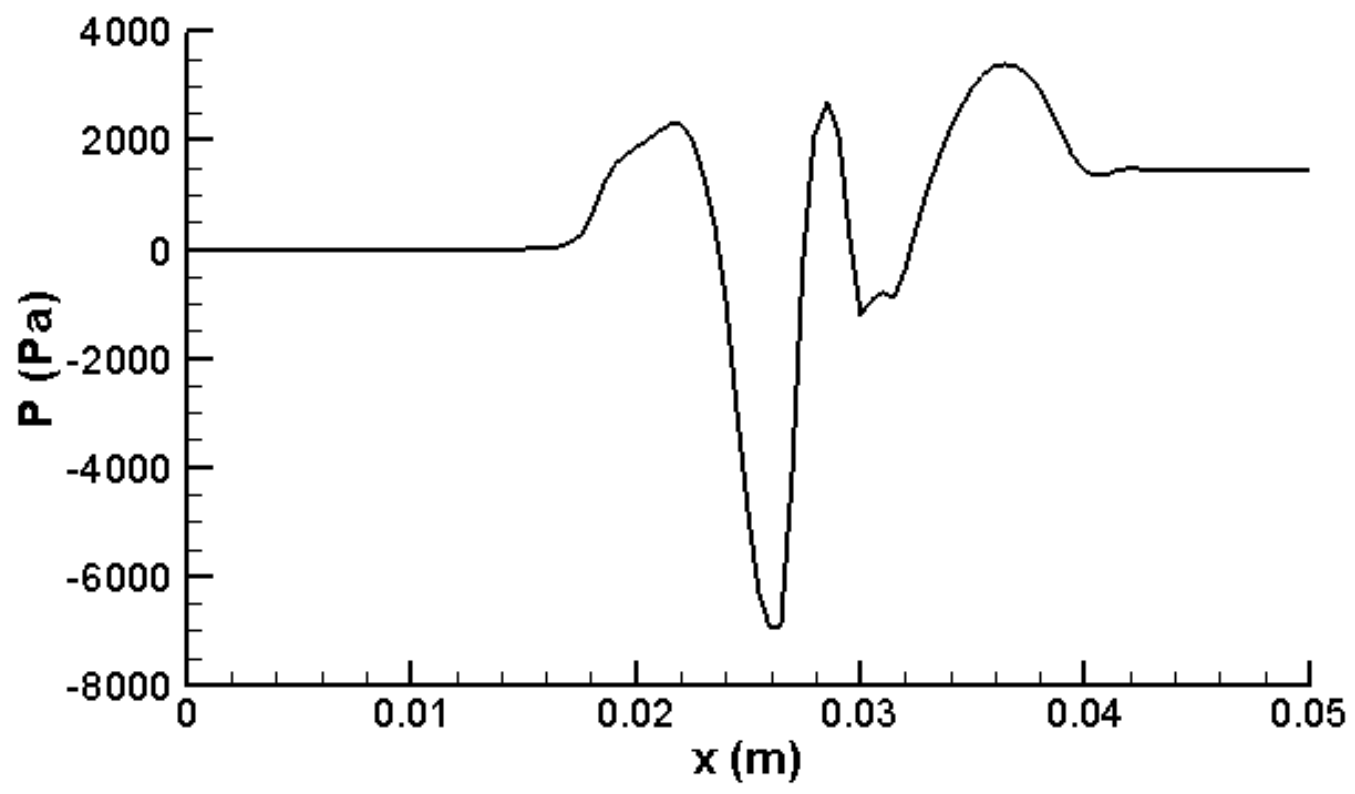

Figure 4.21: Pressure versus axial direction during cough at time $=0.2$ seconds after the cough begins. 


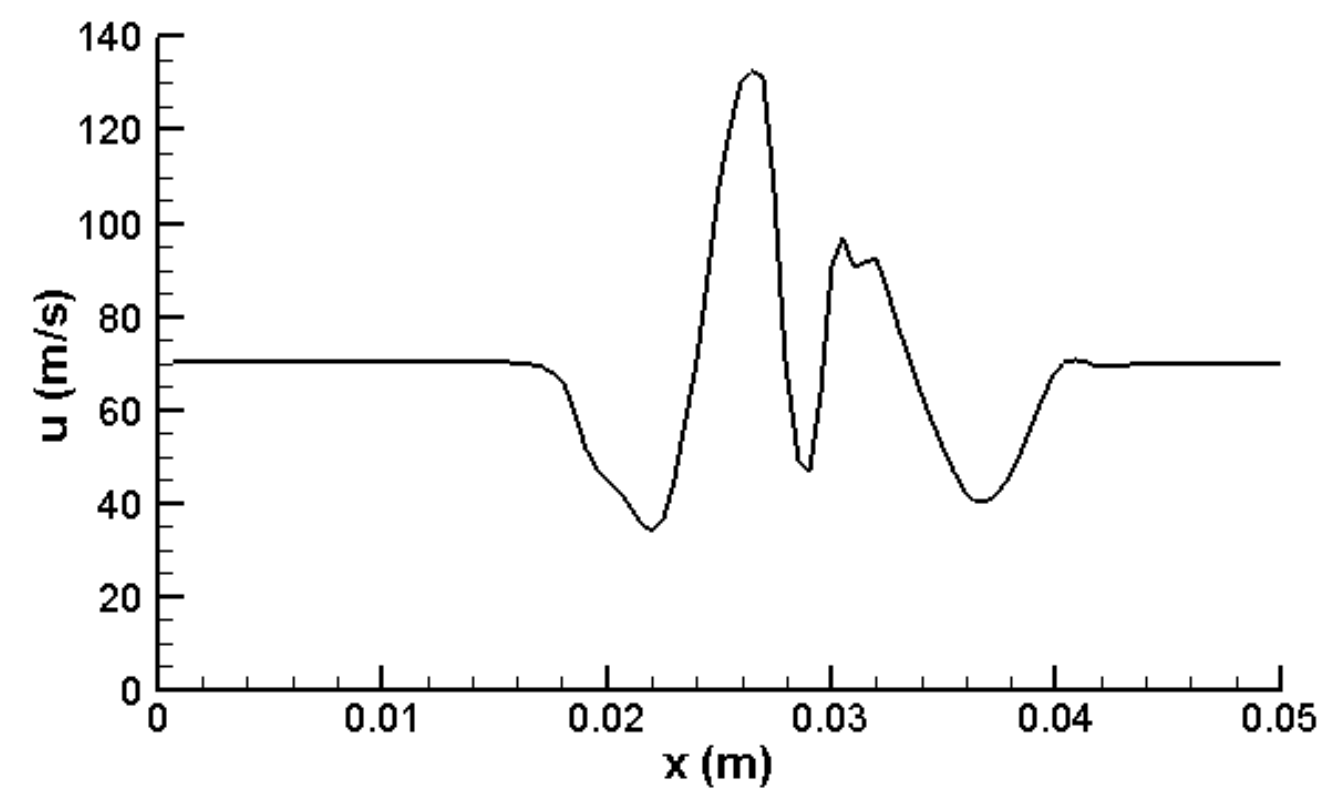

Figure 4.22: Axial mean velocity versus axial direction during cough at time $=0.2$ seconds after the cough begins.

\subsubsection{Three-Dimensional Flow Analysis}

Coughing and particle dispersion during cough is also simulated using FLUENT. The first step in these simulations was to generate a suitable grid for the larynx geometry. Gambit is used to generate such a grid. The three dimensional scan data was modified and refined so that it could be used in Gambit. Some sharp gradients in the geometry are smoothened in such a way that a reasonably smooth grid could have been generated. The surface mesh of the generated 200,000 grid is given in Figure 4.23.

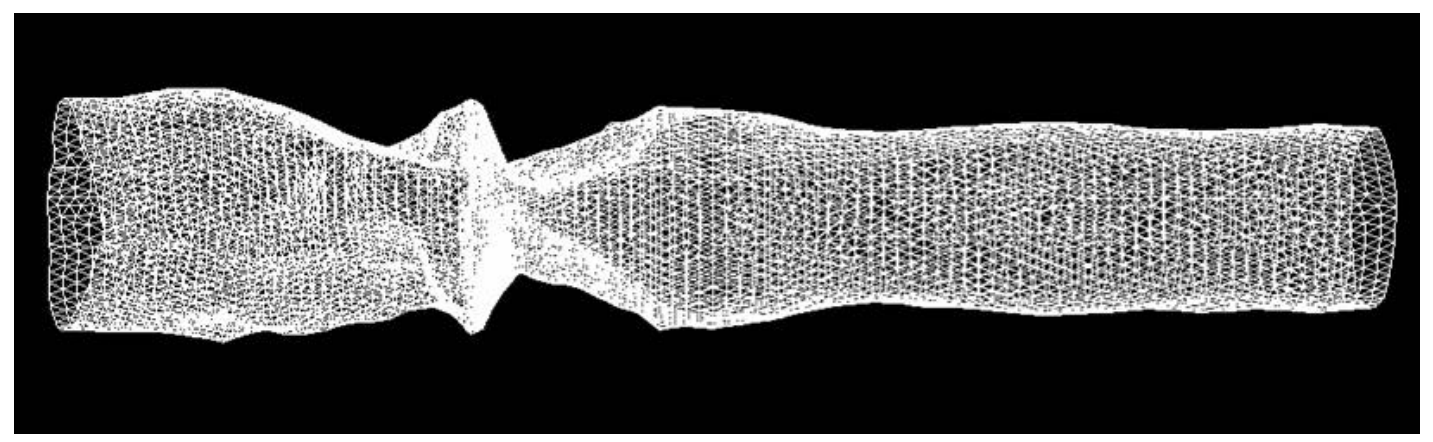

Figure 4.23: 200K Mesh generated by Gambit for FLUENT. 
After generating a grid a trial run is carried out in FLUENT where constant pressure boundary conditions are used on both boundaries. After this trial run, the same boundary conditions are employed as in the PTL model (Fig. 4.19).

RNG $\mathrm{k}-\varepsilon$ model is used as the turbulence model in the cough simulations. The time step is $1 \times 10^{-3}$, and the residuals for mass and momentum are set to $1 \times 10^{-6}$ with maximum inner iterations of 1000 . It is observed that at some time levels it was not possible to achieve this level of convergence. However, the overall accuracy of these simulations are seemed to be sufficient for the purpose.

The pressure contours at the peak of the cough is plotted in Figure 4.24. It can be seen from the figure that there is a significant pressure gradient through the glottis. This behavior is also seen in the onedimensional analysis.

Velocity contours are shown in Figure 4.25. In Figures 4.26 and 4.27, the streamlines and velocity vectors are also shown. The jet formation can easily be seen from these figures downstream of the glottis.

The turbulent kinetic energy is plotted in Figure 4.28. There are some regions where the turbulent kinetic energy has significantly large values although it is almost zero in most of the regions. This appears to be a good representation of the transitional flow regime from laminar to turbulent in the larynx. 


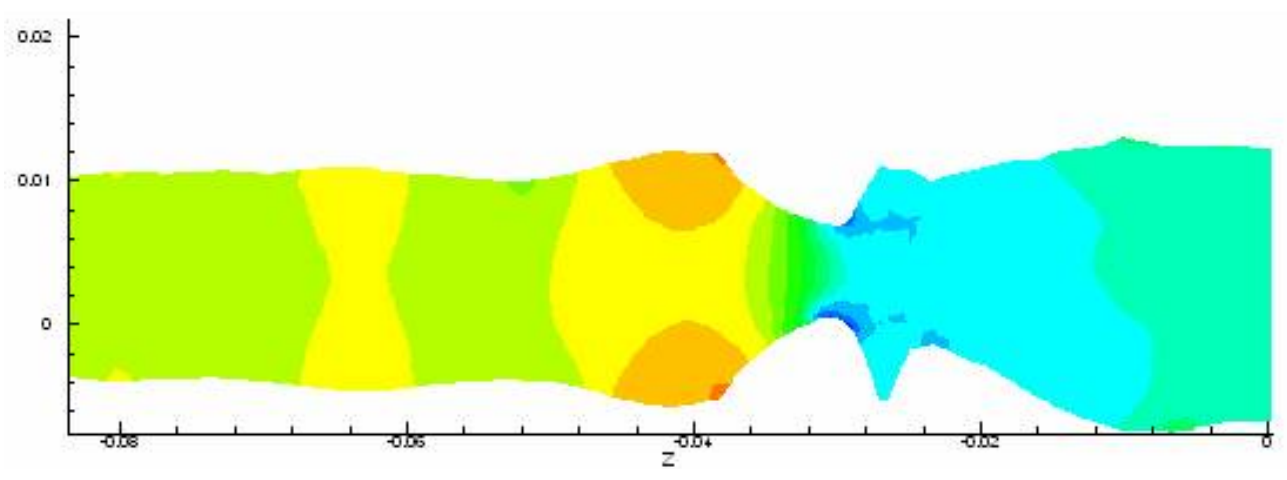

Figure 4.24: Pressure contours at the peak of the cough.

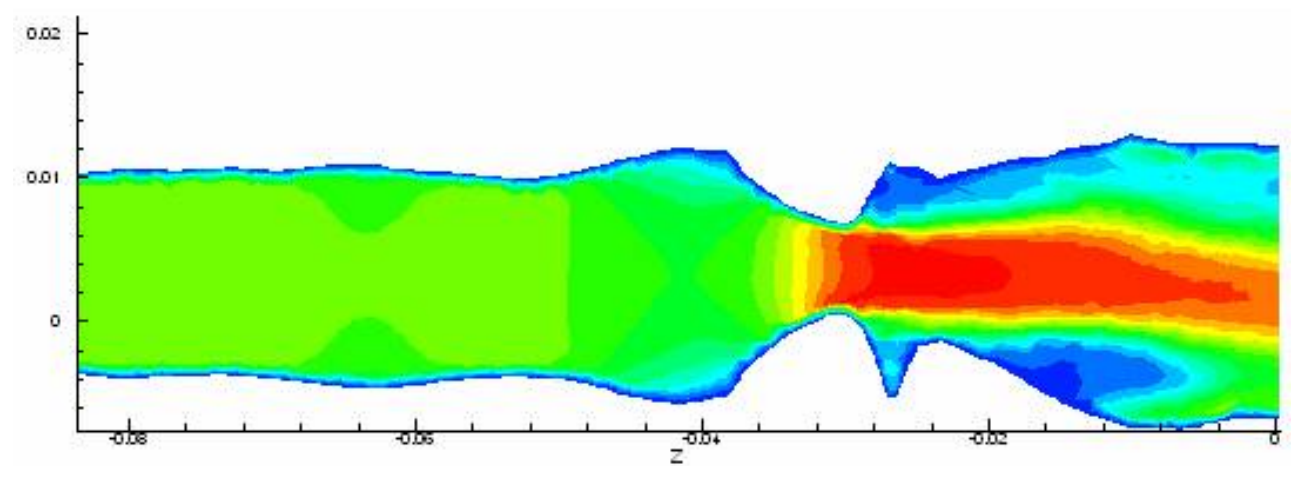

Figure 4.25: Velocity contours at the peak of the cough.

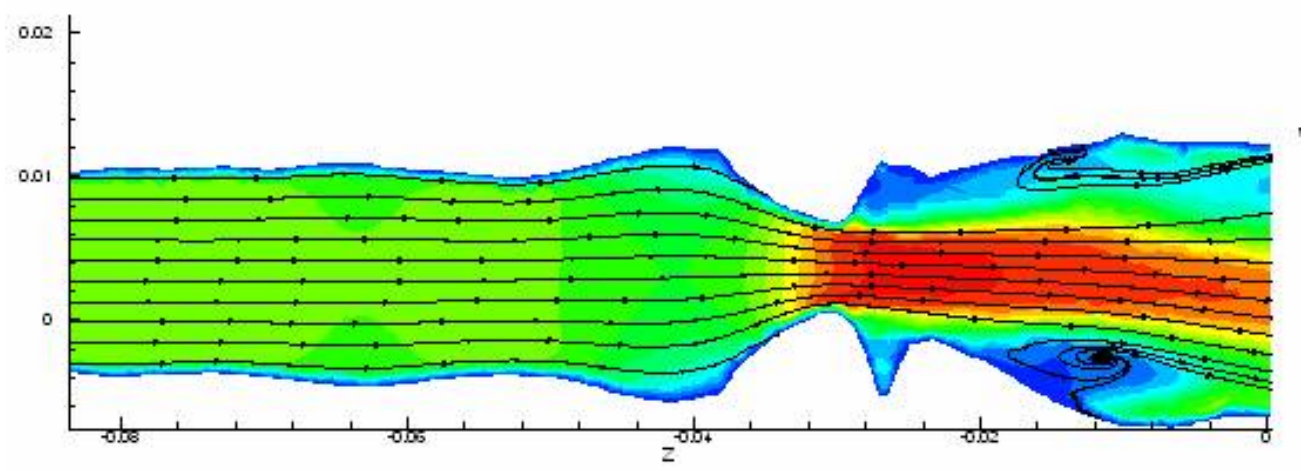

Figure 4.26: Velocity contours and streamlines at the peak of the cough. 


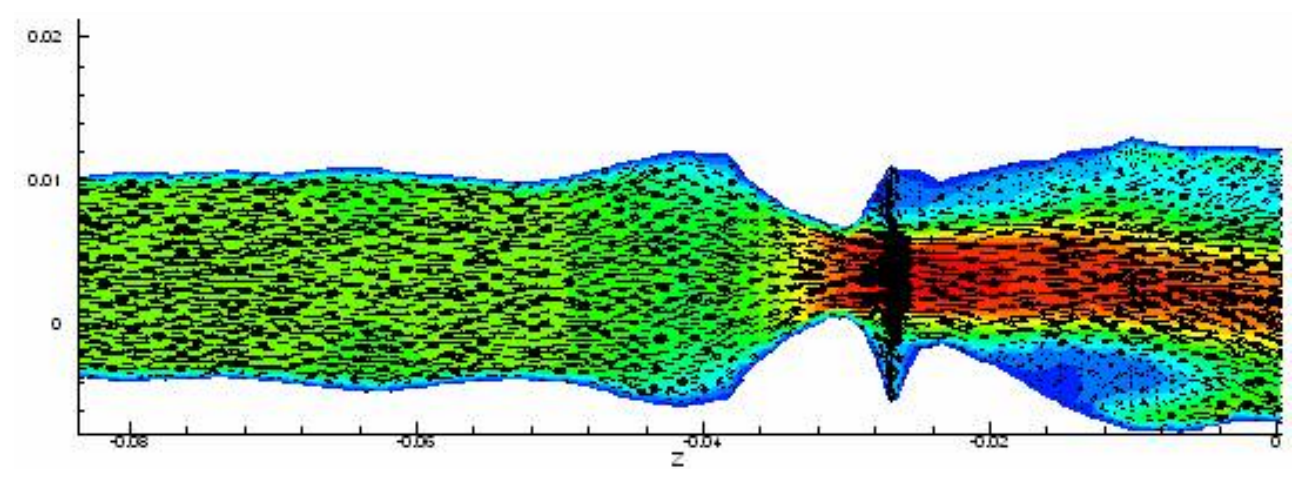

Figure 4.27: Velocity contour and velocity vectors at the peak of the cough by FLUENT.

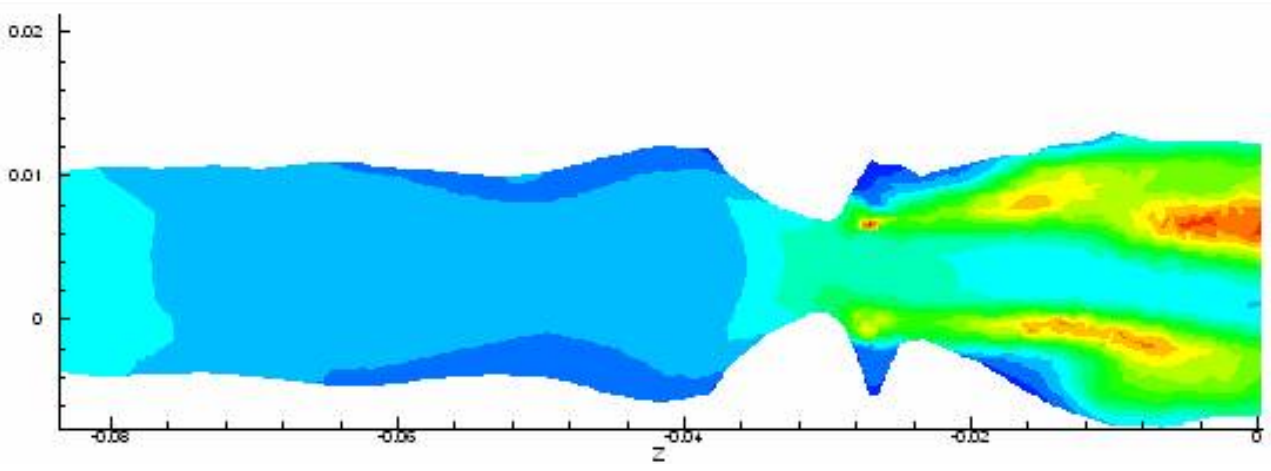

Figure 4.28: Turbulent kinetic energy at the peak of the cough by FLUENT.

The flow field is recorded at certain axial locations during the simulations. The locations, at which the two-dimensional velocity field is recorded and the streamlines with velocity contours at these locations, are depicted in Figure 4.29. 


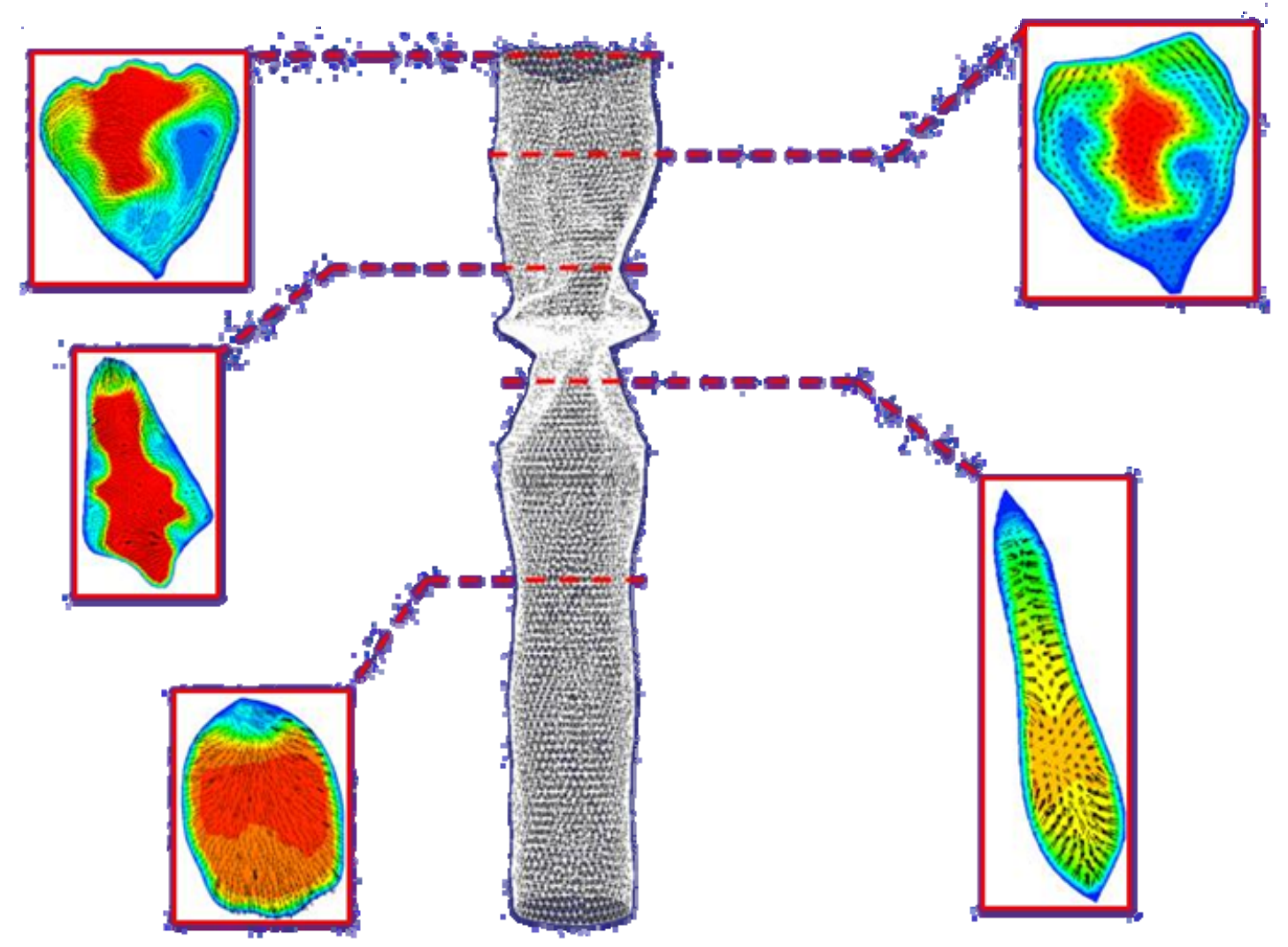

Figure 4.29: Streamlines with velocity contours at the given axial locations.

Figure 4.29 shows that the flow is quite regular upstream of the glottis but is very complicated with many recirculation regions observed downstream of the glottis.

A further step in this simulation is the injection of the particles to the system and recording the deposition patterns during the exhalation process. The injections of the particles are carried out in such a way that they represent the particles which will be entrained during the cough. For this purpose four lines are defined in the FLUENT in the subglottic region at $90^{\circ}$ apart from each other and the injection took place only until the peak of the cough and then it was stopped. In a more detailed study, these lines may be replaced by a surface, which covers the whole subglottic region, to represent 
a more realistic entrainment, and the injections may be defined as given in the proposed droplet model. The physical properties of the particles and the injection parameters are given in Table 4.1 and Table 4.2., respectively.

Table 4.1. Physical properties, of the particles injected.

\begin{tabular}{cl} 
Property & \multicolumn{1}{c}{ Value } \\
\hline \hline Particle Type & Quartz \\
Particle Density & $2650 \mathrm{~kg} / \mathrm{m}^{3}$ \\
Particle Diameter & $1 \mu \mathrm{m}$ \\
\hline
\end{tabular}

Table 4.2. I njection properties.

\begin{tabular}{cl} 
Parameter & \multicolumn{1}{c}{ Value } \\
\hline \hline Injection Rate & $1 \mathrm{mg} / \mathrm{s}$ \\
Injection Period & $0.012 \mathrm{~s}$ \\
Total Injection Mass & $12 \mathrm{mg}$ \\
\hline
\end{tabular}

In the simulations all the particles that hit the wall are assumed to be depositing on the wall. According this assumption it is found that circa half of the particles left the system whereas, the other half deposited on the walls of the respiratory tract (Table 4.3).

Table 4.3. Results of particle tracking from FLUENT simulations.

\begin{tabular}{cc} 
Property & Value \\
\hline \hline Injected Particles & 920 \\
Particles Left & 465 \\
Deposited Particles & 454 \\
\hline
\end{tabular}




\subsection{Kinetic Energy Calculations}

The kinetic energy equation is solved in a pipe flow by both PTL model and FLUENT. The pipe radius is taken as the larynx radius at the largest cross section. The calculated kinetic energy from the PTL model is compared with the results obtained from FLUENT on various planes at different axial locations. Then the three-dimensional FLUENT results are integrated over the cross-sectional area perpendicular to the axial direction to obtain an average kinetic energy. The PTL model coefficients are tuned to match the FLUENT results. The comparison is done at three different Reynolds numbers and two of these comparisons are plotted in Figure 4.30.

After the model coefficients are tuned to match the cases mentioned above they are fixed at those values through out the study. This tuning is done in such a way that the best match is encountered at higher Reynolds numbers. In this way the turbulent kinetic energy prediction has higher accuracy at higher Reynolds number, where we encounter more entrainment. The PTL model coefficients used are given in Table 4.4.

Table 4.4. Model coefficients used in turbulent kinetic energy equation.

\begin{tabular}{clc} 
Variable & \multicolumn{1}{c}{ Definition } & Value \\
\hline \hline$\sigma_{\text {tur }}$ & Turbulent model coefficient & 1 \\
$C_{v}$ & $\begin{array}{l}\text { Turbulent viscosity model } \\
\text { coefficient }\end{array}$ & 0.5 \\
$\ell_{\mathrm{t}}$ & Turbulent length scale & $0.1 \mathrm{R}$ \\
$C_{D}$ & Dissipation model constant & 2 \\
\hline
\end{tabular}




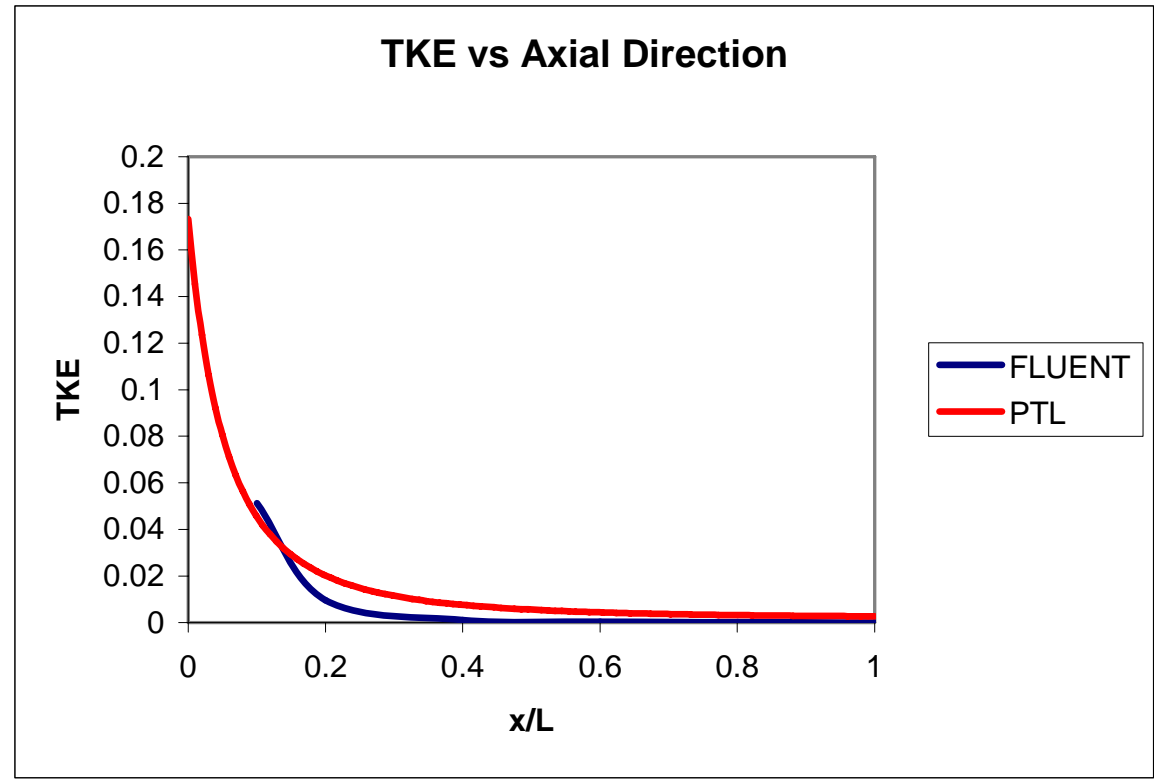

(a)

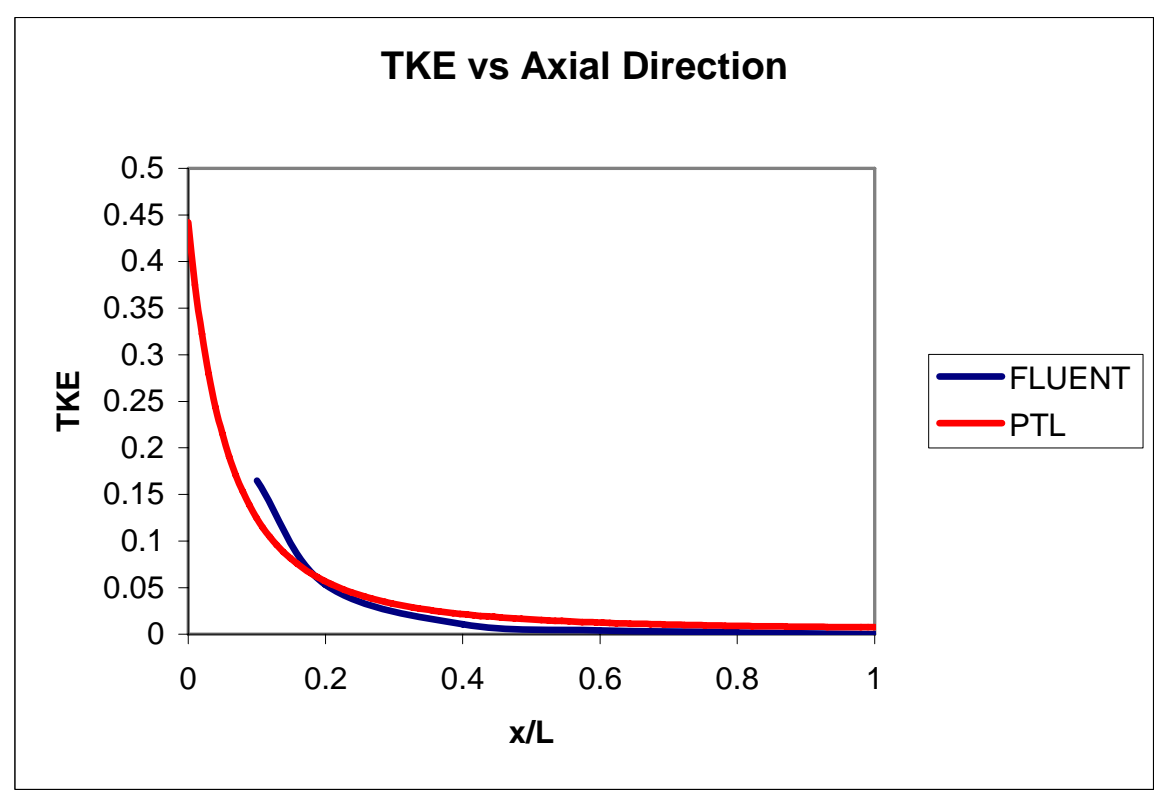

(b)

Figure 4.30: Comparison of turbulent kinetic energy obtained from the model and FLUENT at a) $R e=2400$; and b) $R e=3800$. 


\subsection{Aerosol Generation}

The aerosol generation model is integrated with the one-dimensional flow solver through the solution of the turbulent kinetic energy equation. The model constants in the turbulent kinetic energy equation are tuned by comparison with the FLUENT results. The tuning of the model coefficients in the aerosol generation and entrainment model are done with the only experimental data available in hand. The coefficients are adjusted in such a way that the final aerosol size distribution matches the experimental measurements. The experimental data is given in Figure 4.31.

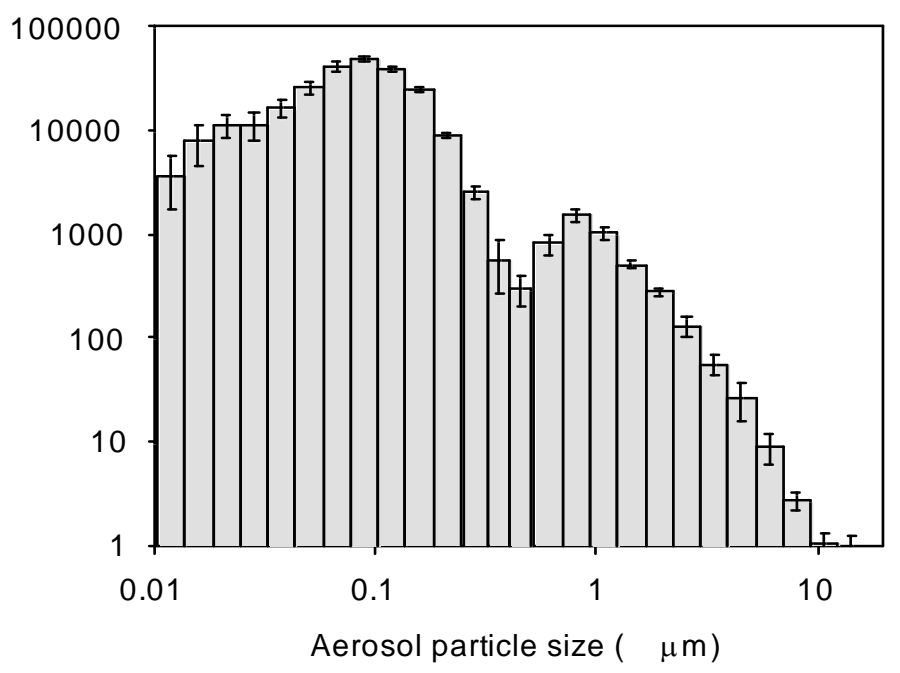

Figure 4.31: Measured averaged aerosol size distribution during cough (The vertical axis is the concentration of aerosol) [5].

The aerosol size distribution obtained by the proposed model is given in Figure 4.32. It can be from the figures that, the model can produce similar results to the experimental measurements. 


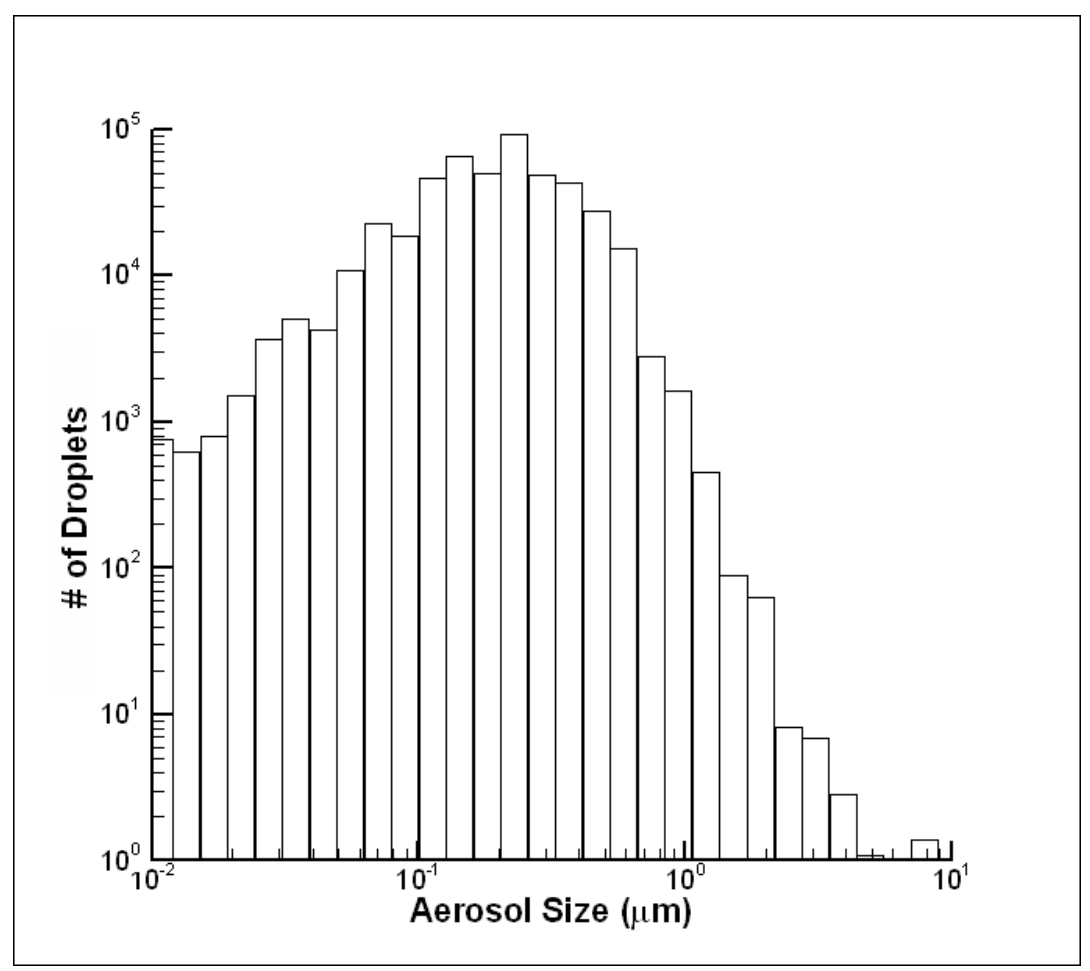

Figure 4.32: Averaged aerosol size distribution during a cough.

The aerosol size distribution obtained by the model given above is the average of the twelve different aerosol distributions for twelve different cough signals, excluding subject three (Figure 4.17). In order to see the effects of different cough signals, three different size distributions is given for three different coughs with their average aerosol size in the following figures (Figure 4.33). 


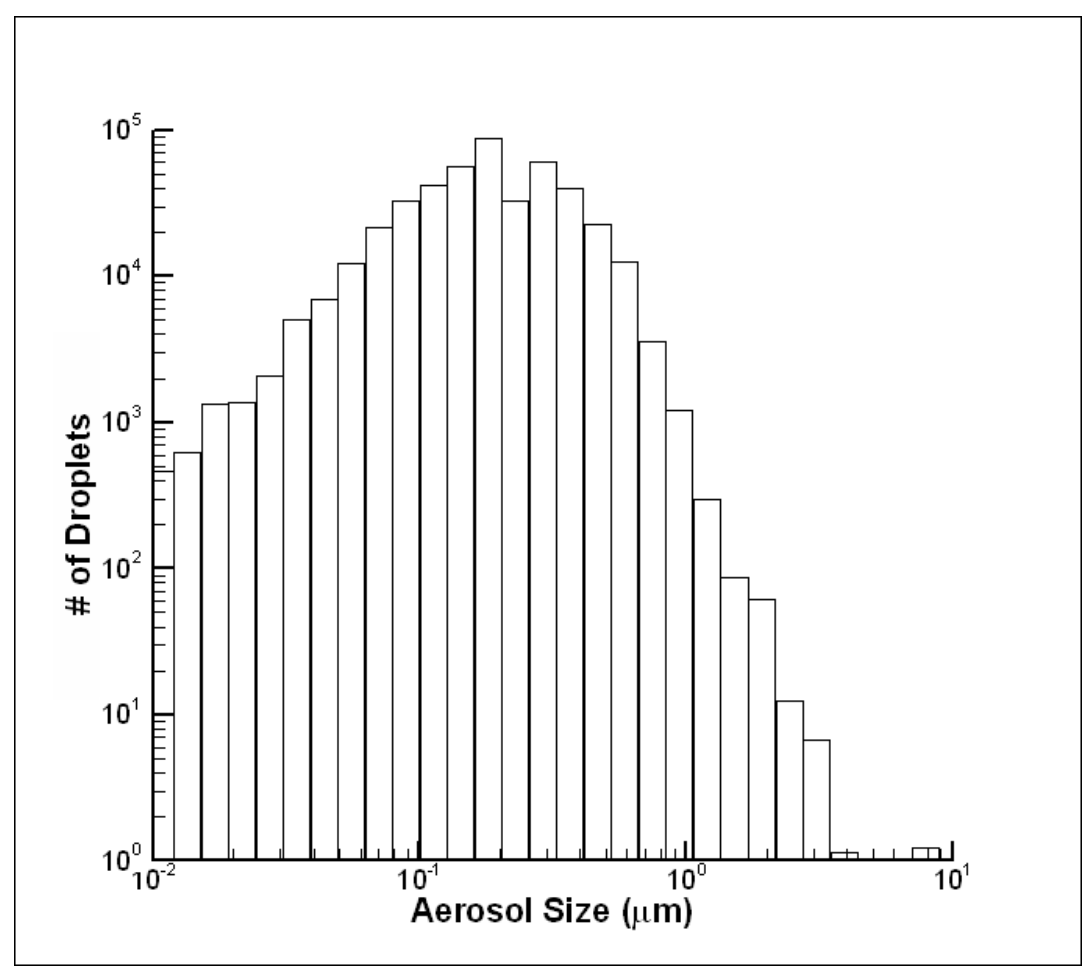

(a)

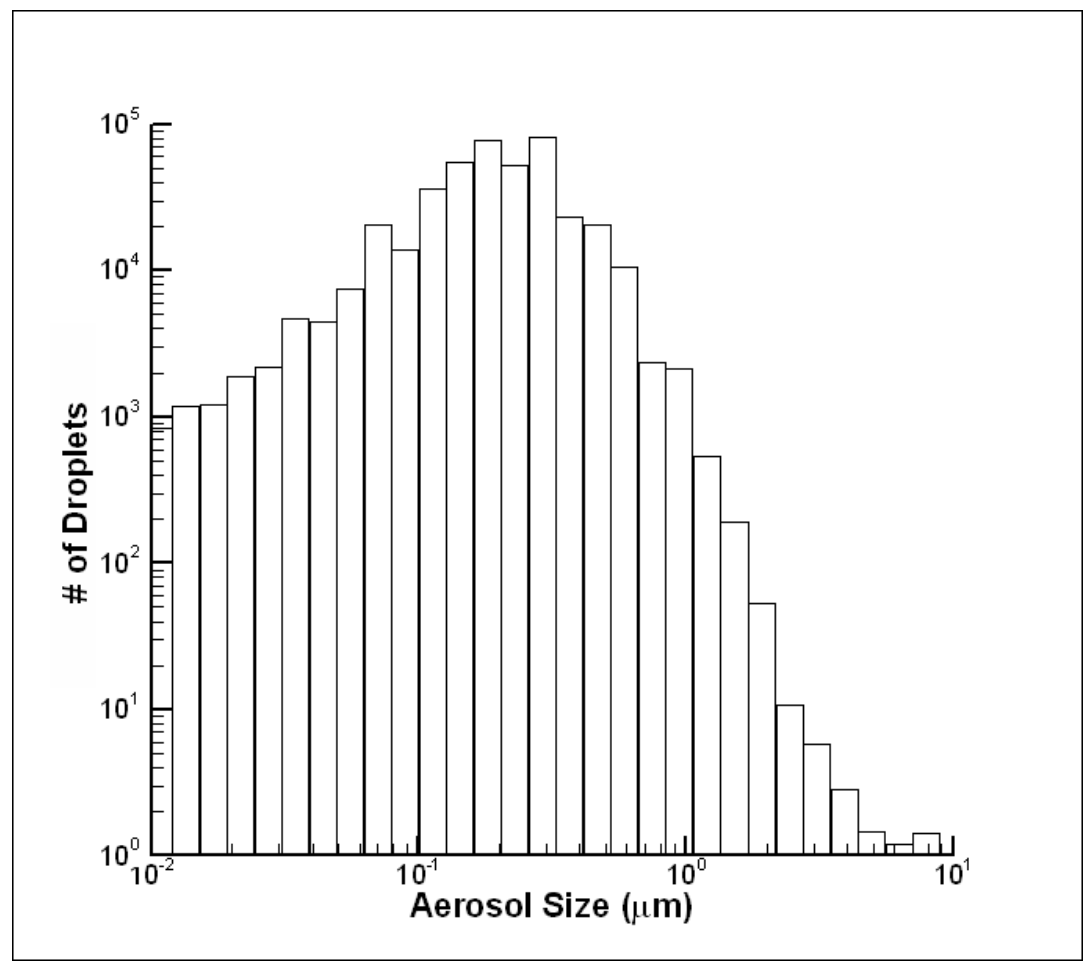

(b) 


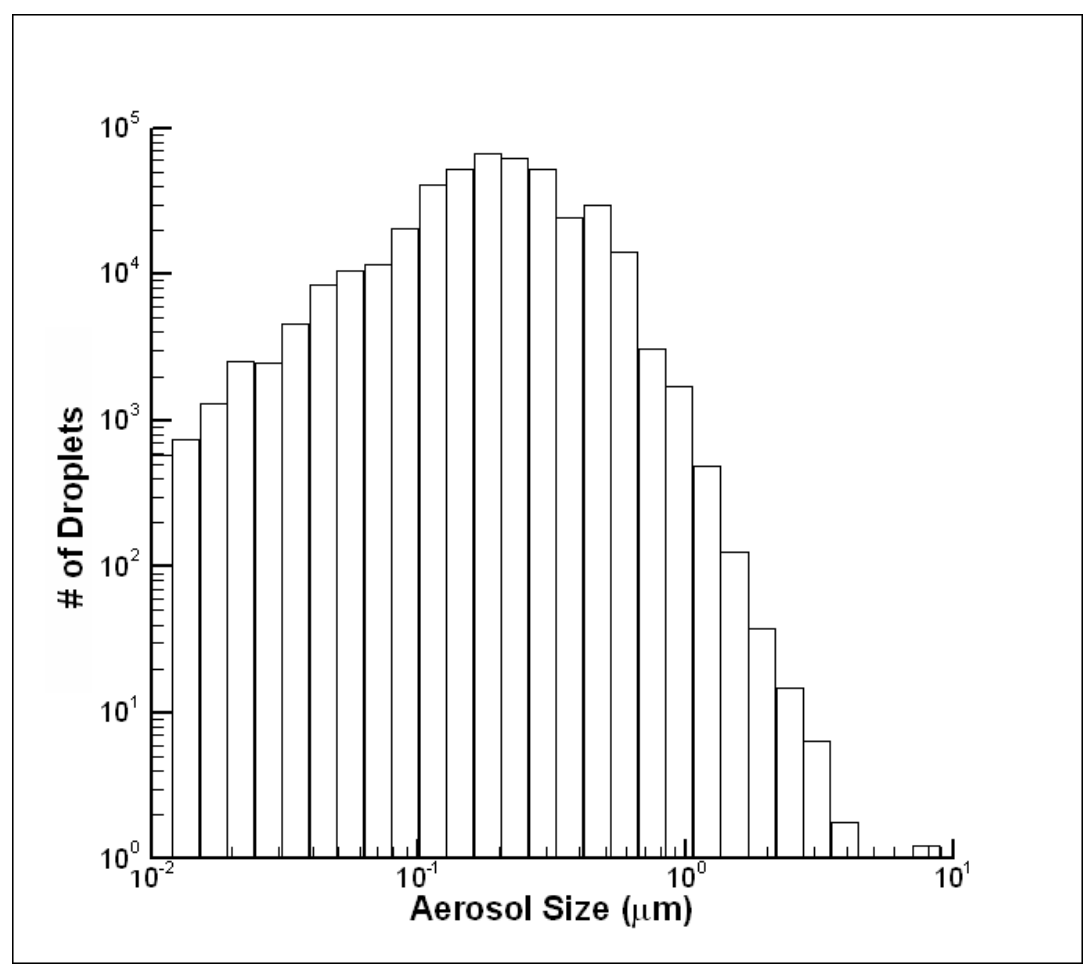

(c)

Figure 4.33: Aerosol size distribution during, (a) second cough of first subject; (b) first cough of fourth subject; (c) third cough of fifth subject.

Since these cough signals were approximately equal to each other, it was expected to obtain similar results for each cough signal. All three simulation results for three different coughs are plotted in Figure 4.34, for a better comparison. The random function, used in the binary droplet breakup mechanism, also has an effect on this similarity but not on the exact size distribution pattern. It should be noted that, for the same coughing signal it is possible to obtain slightly different aerosol distribution every time the simulation is done. In Figure 4.35, the difference of the aerosol size distribution among three cases is given. In this figure the vertical axis is given as a difference between the numbers for each cough and the average droplet size distribution. Figure 4.35 shows clearly that each cough cycle generates a different aerosol distribution. 


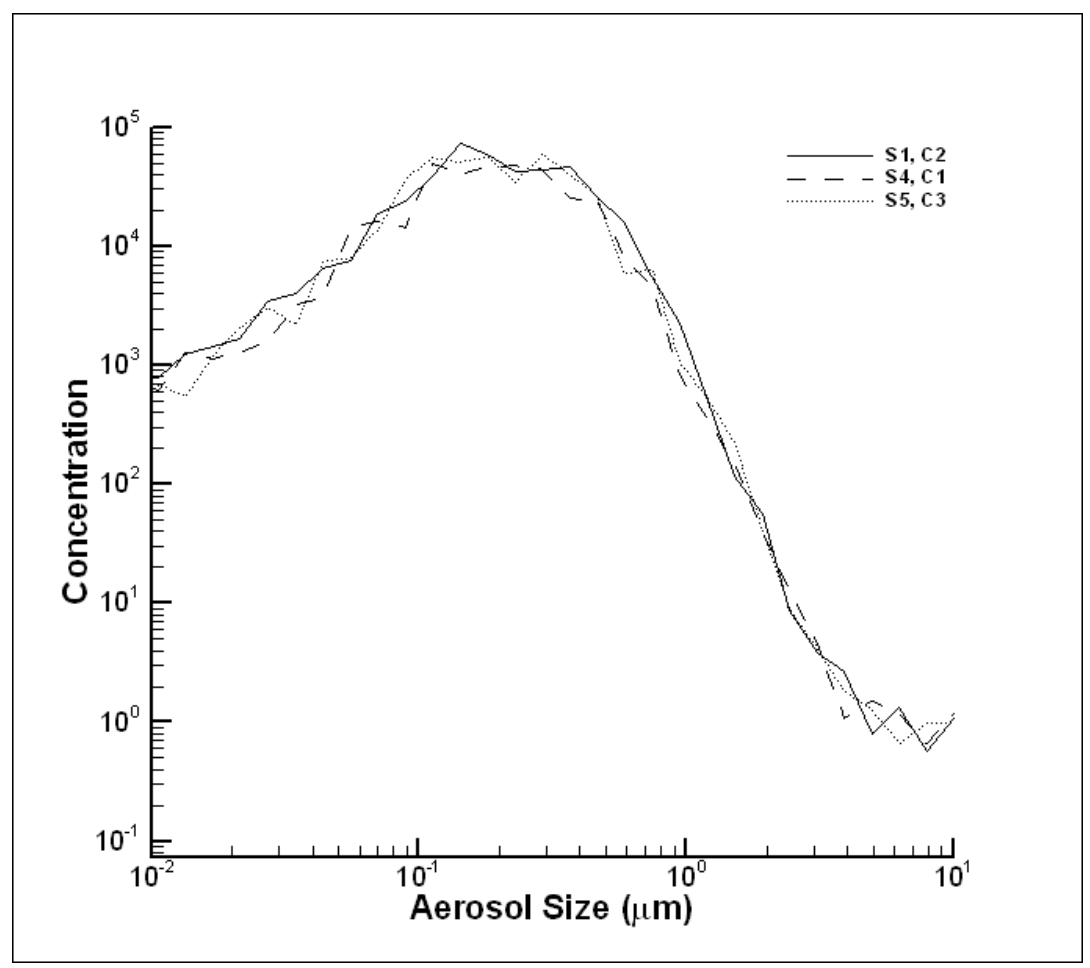

Figure 4.34: Aerosol size distribution for all three coughs.

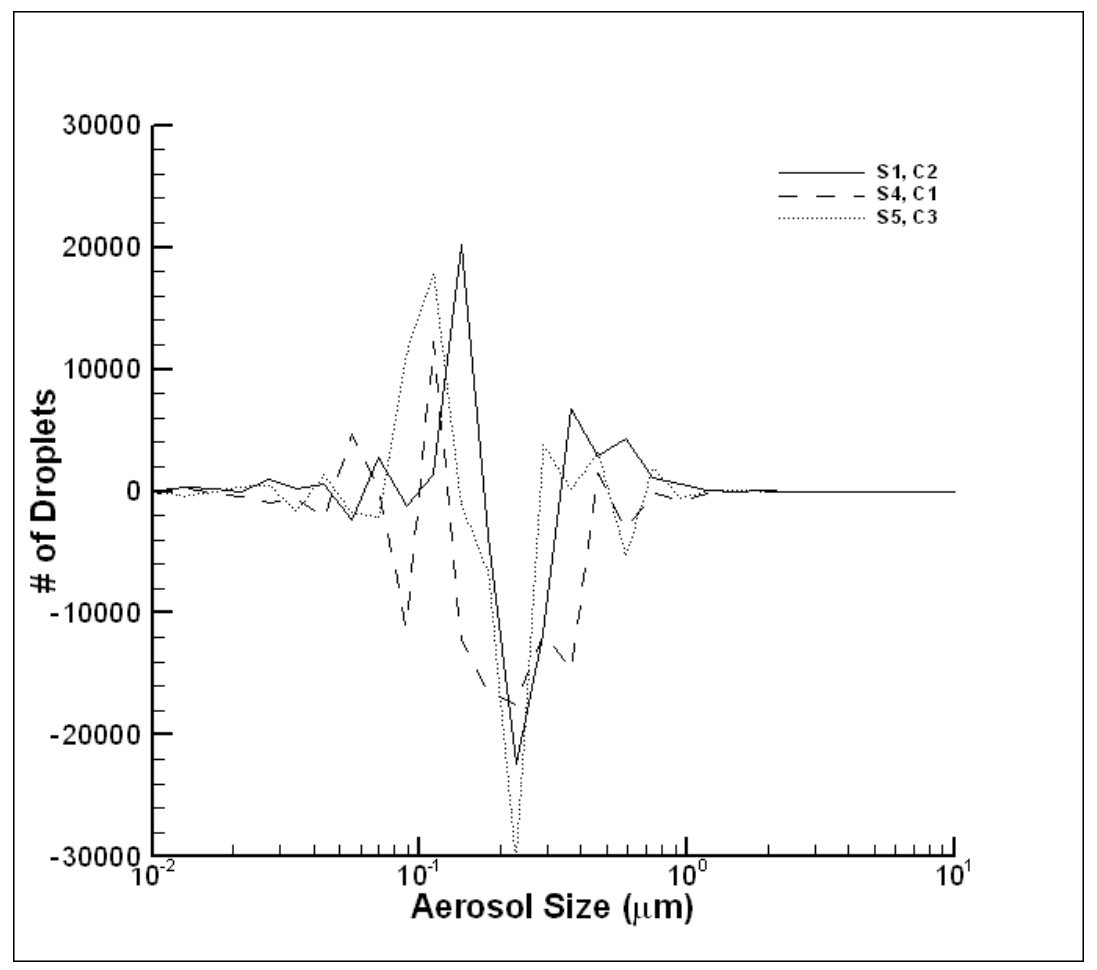

Figure 4.35: Difference between the aerosol size distribution for all three coughs and the average size distribution. 
In order to see the effect of random function during the break up process, the same cough signal is used three times to simulate the same cough. The aerosol size distribution curves for these three consecutive coughs are given in Figures 4.36 and 4.37. Figure 4.36 shows the three distribution curves for these three runs, while Figure 4.37 shows the difference between the individual distribution and the average size distribution. As it can be seen form the figures, the random function has an effect on the size distribution of the aerosols as expected. This effect in addition to the variation in the cough signal adds up to produce slightly different distribution patterns from cough to cough as shown in the previously.

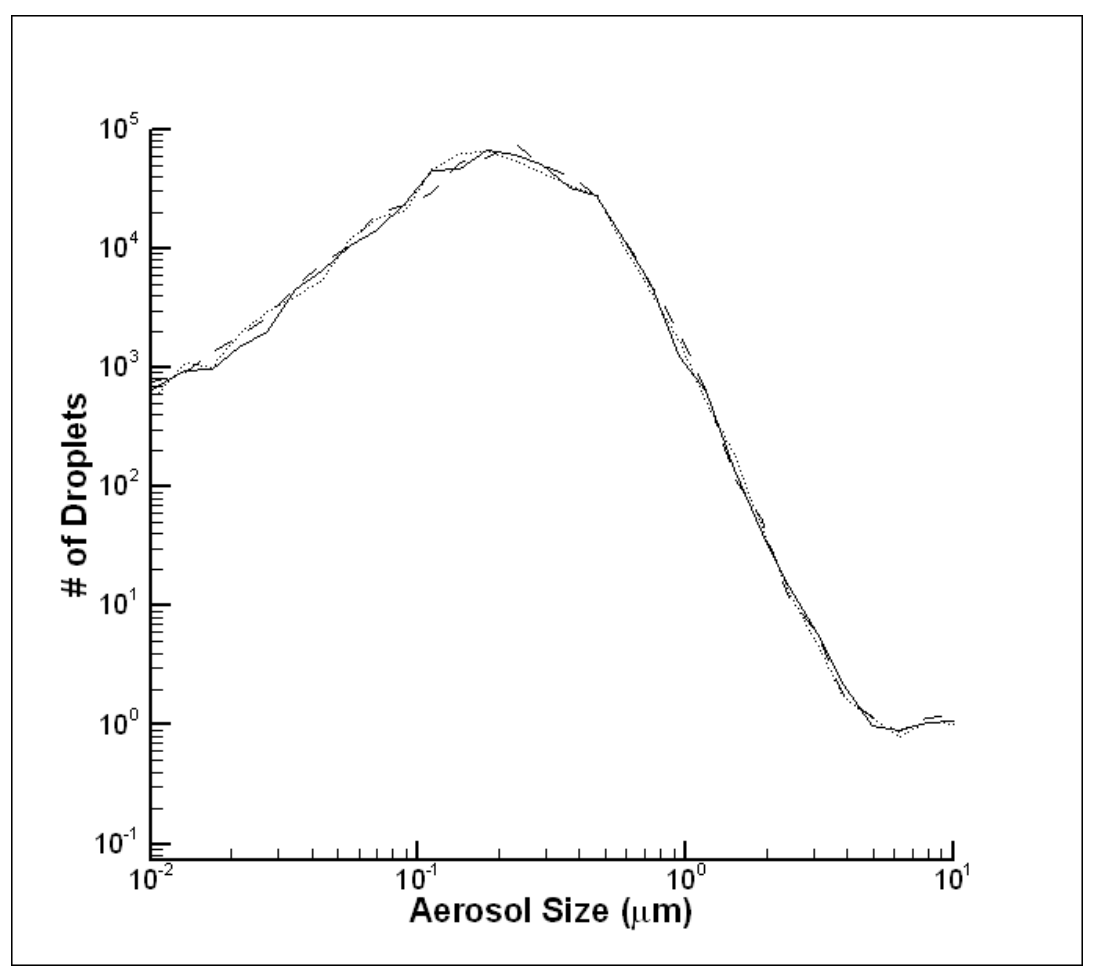

Figure 4.36: Effect of random function on the same cough signal. 


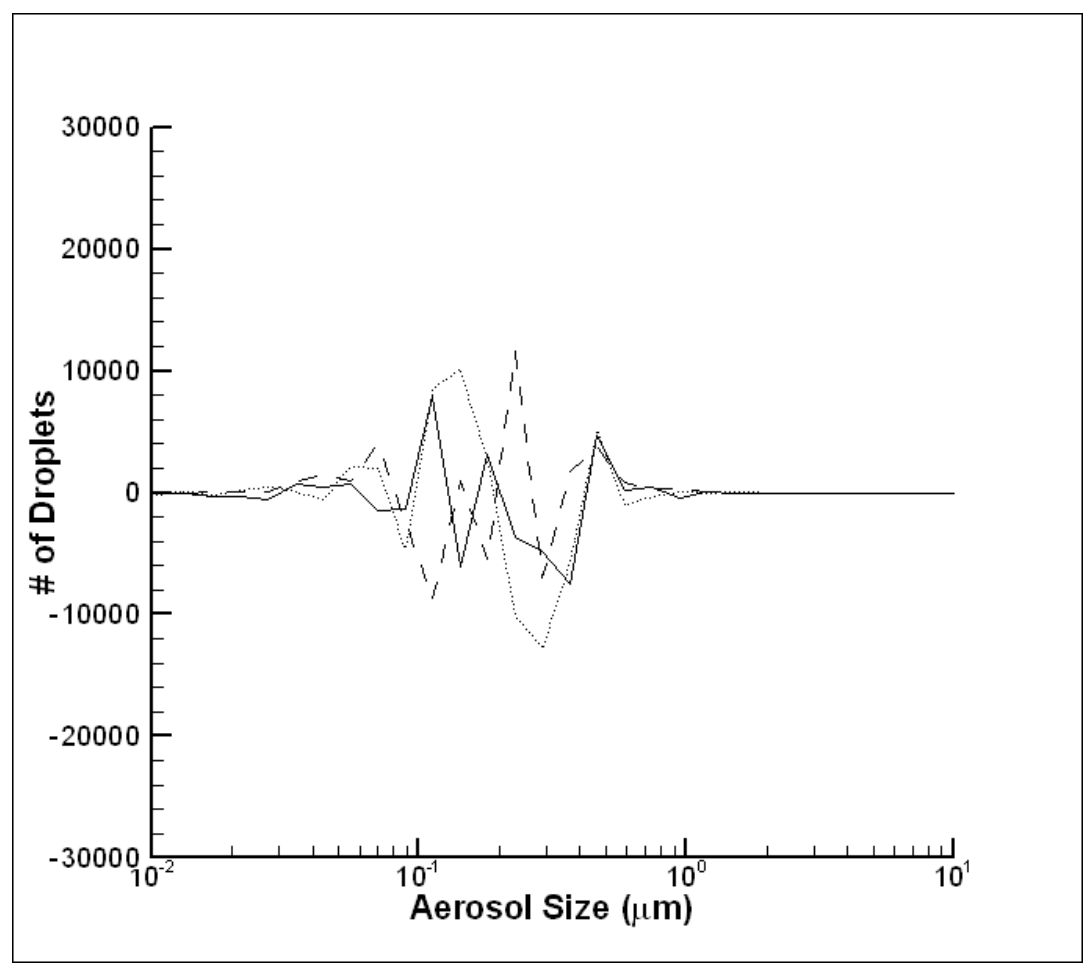

Figure 4.37: Difference between the aerosol size distribution for the same cough in different runs.

One of the subjects (Subject three, Figure 4.17) used in the experimental measurements had a health condition and produced a different cough signal than the other subjects. Since this signal was far from the others, it was not included in the averaging process in order to keep the standard deviation as low as possible. However, this cough signal is also simulated and the aerosol size distribution obtained is shown in Figure 4.38.

Since the strength of the cough is not as strong as the others, it was expected that a much less number of aerosol would be generated with a size distribution shifted towards bigger droplets since the turbulent kinetic energy would be expected lower in this case than the others. This expected result can be seen in the figure clearly. 


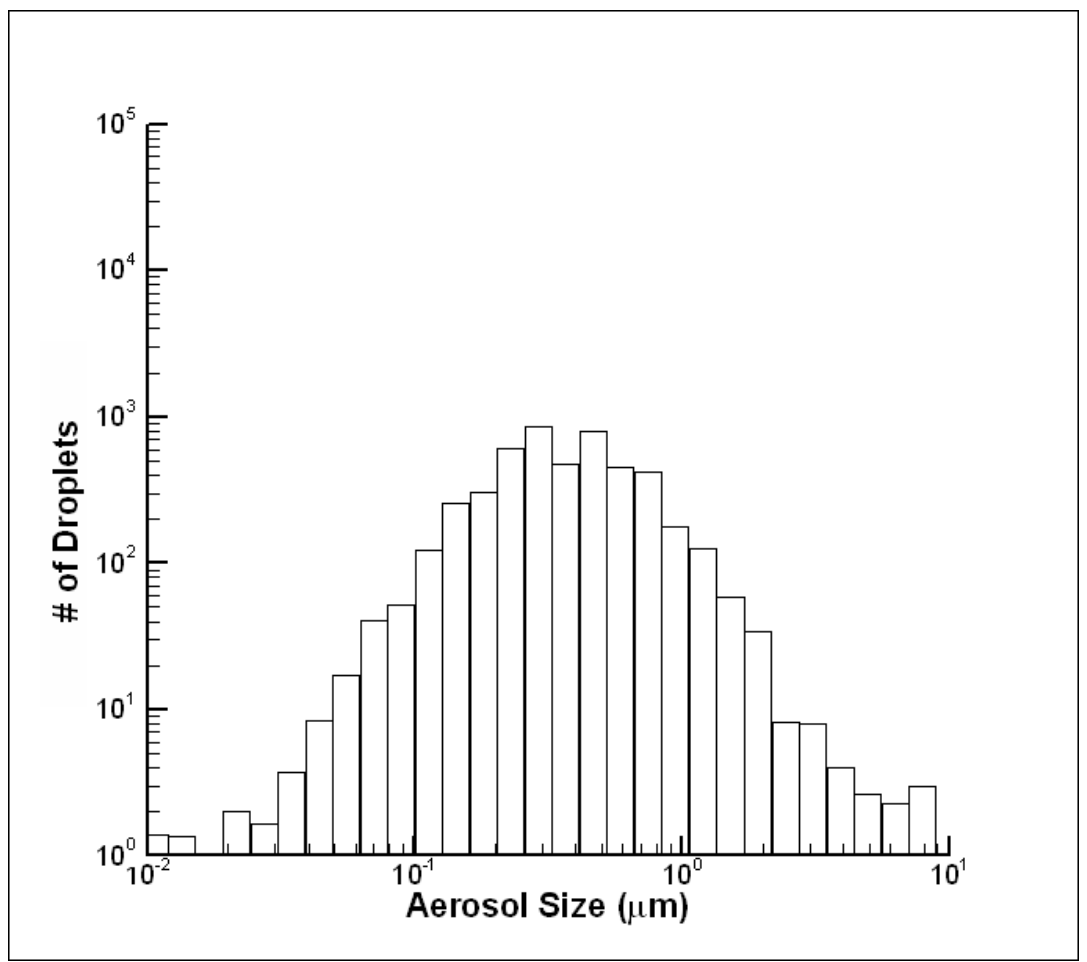

Figure 4.38: Aerosol size distribution during first cough of third subject.

A further step in this study is to investigate some parametrical study. The effect of the physical properties of the mucus is investigated and it is found that, the effect of the surface tension is the largest among the other physical properties. The density, viscosity and surface tension has been varied in the simulations and it has been noted that the effect of density and viscosity is much less than that of surface tension. In Figure 4.39., the aerosol size distribution is given for three different values of the surface tension.

As it can be seen from the figures, the surface tension effects the distribution significantly. And this effect is in the direction of the expectations. When the surface tension decreases, the number of aerosols 
increases since it is easier for them to break, and the size distribution shifts left, which gives smaller aerosol size distribution. When the surface tension increases, the opposite effect is observed.

The reason for the density and viscosity having less effect on the final result can be explained as follows. The density mainly goes into the calculations in the break-up frequency equations, and compared to the other terms in this equation in does not have too much effect on the final result. Similarly, viscosity goes into the equations in the critical shear stress calculations as a ratio to the viscosity of air. Since the ratio is already small, the change in the viscosity does not have a big impact on the final result.

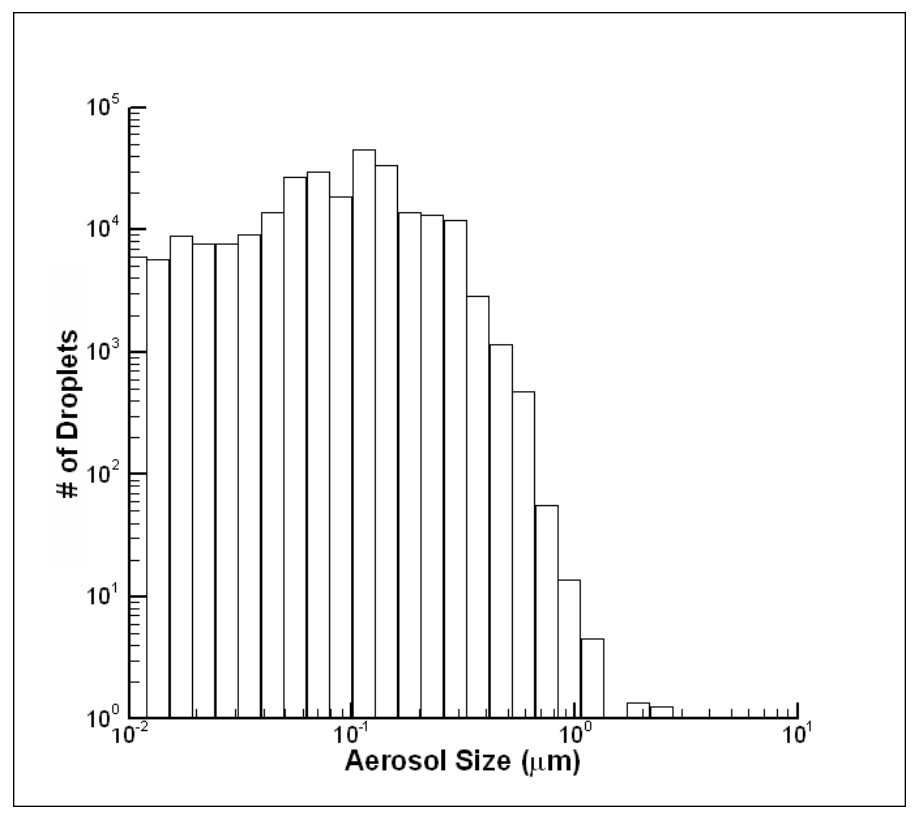

(a) 


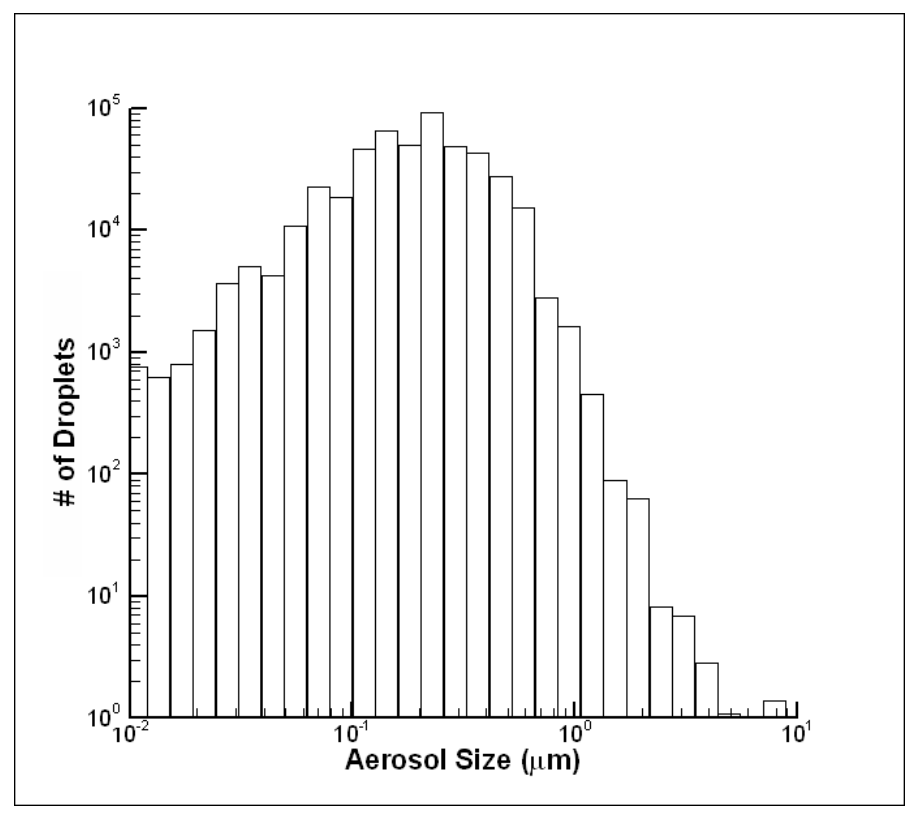

(b)

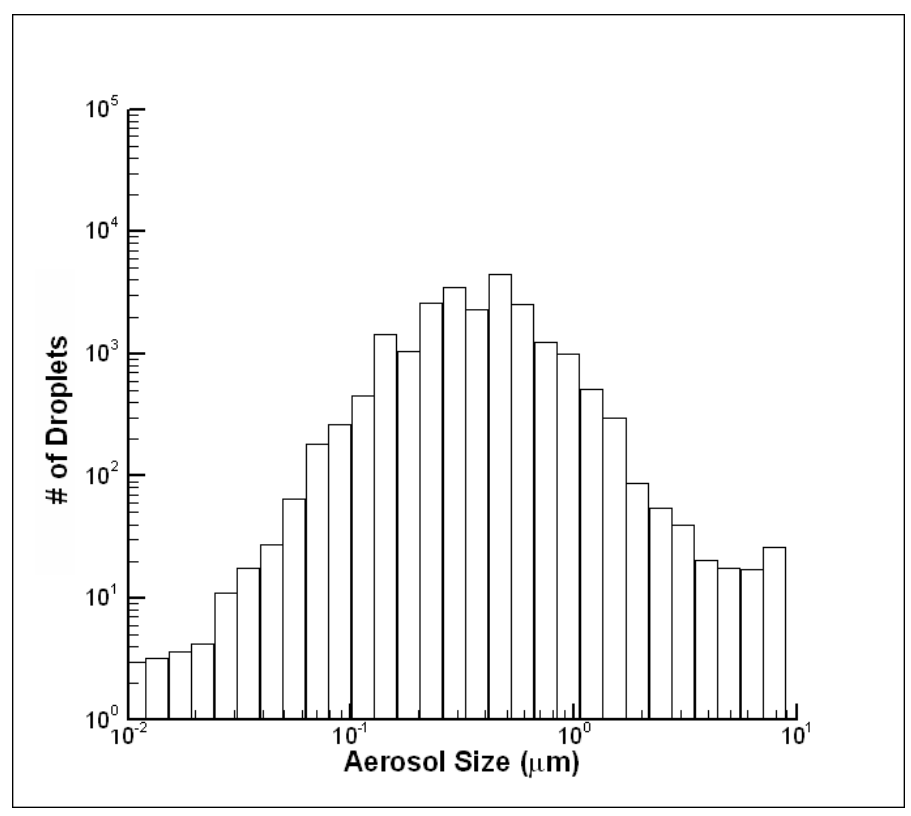

(c)

Figure 4.39: Aerosol size distribution for various surface tension values. (a) $0.1 \sigma$, (b) $\sigma$ and (c) $10 \sigma$. 


\subsection{Consecutive Coughing}

Several consecutive coughs are simulated. In this rapid consecutive coughing, there is no breathing period between the coughs. Two different types of cough cycles have been simulated. The boundary conditions used for these simulations are given in Figures 4.40 and 4.41 . In the first one the same cough signal is repeated four times (Figure 4.40) and in the second one the magnitude of the cough is decreased from one cough to another (Figure 4.41).

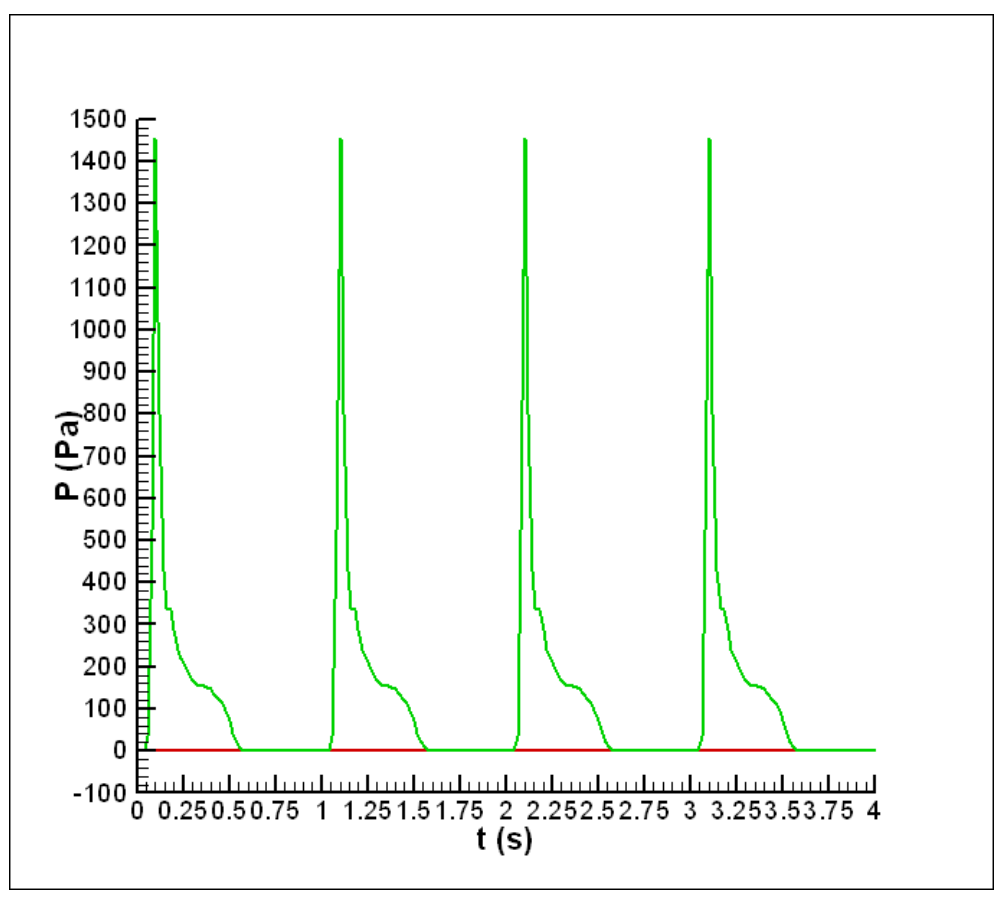

Figure 4.40: Boundary condition for consecutive coughs (same strength). 


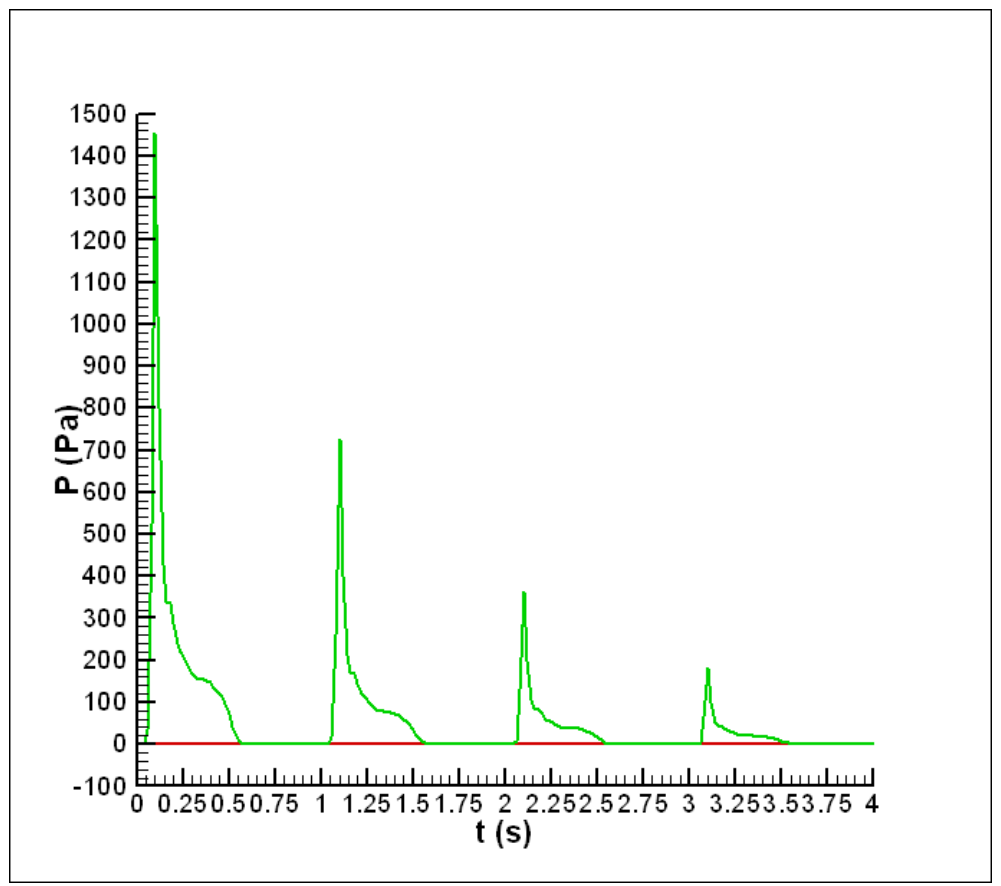

Figure 4.41: Boundary condition for consecutive coughs (decreasing strength).

The size distribution of the aerosol generated after these coughs are given in Figures 4.42 and 4.43. In the first case, where the same cough signal is repeated four times, almost the same size distribution is obtained with a shift in the number of droplets for each aerosol size (Fig. 4.42). However, during the second case where the cough strength decreases from cough the cough, the size distribution shifts towards to larger aerosol size (Fig. 4.43). This is expected, since it has been shown that as the cough strength decreases the aerosol size distribution shifts toward larger sizes. Therefore in Figure 4.43 the effect of both the strong and weak coughs can be seen. 


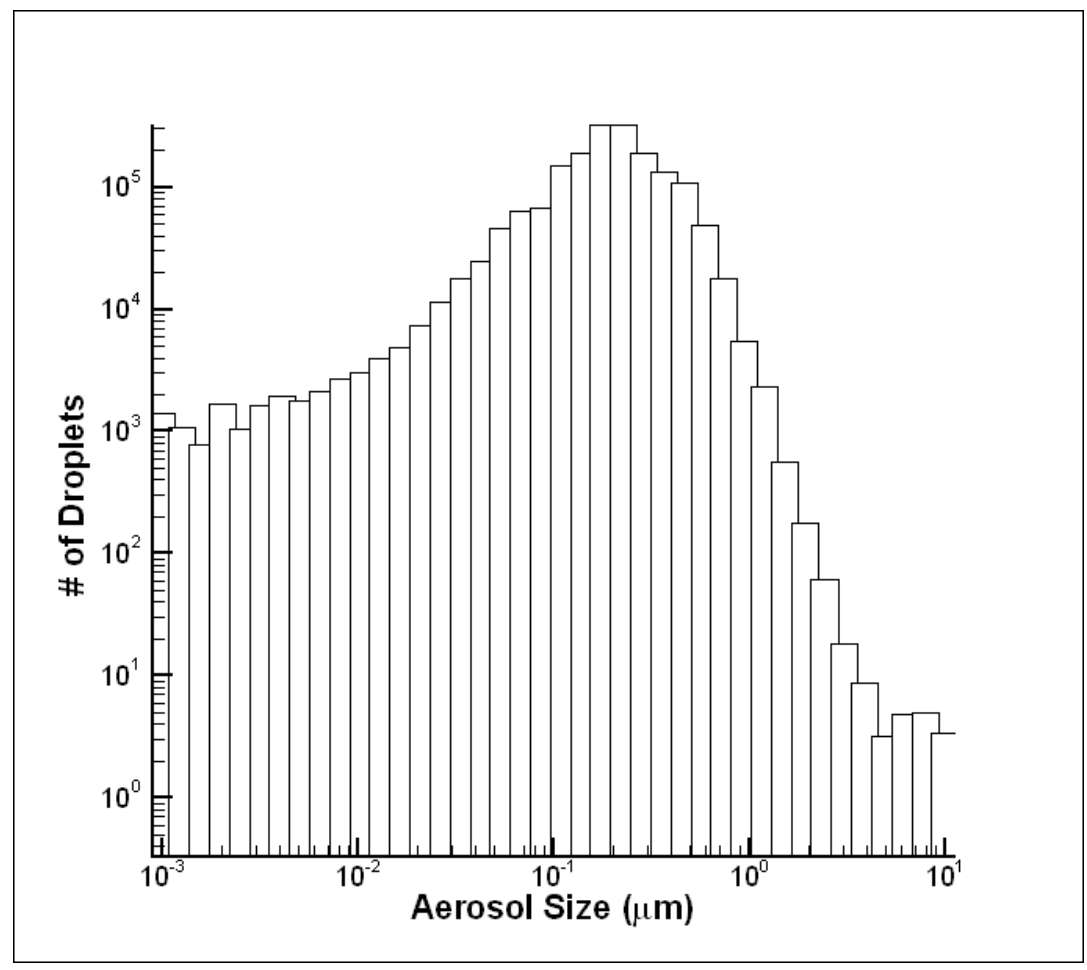

Figure 4.42: Accumulated aerosol size distribution after coughs (same strength). 


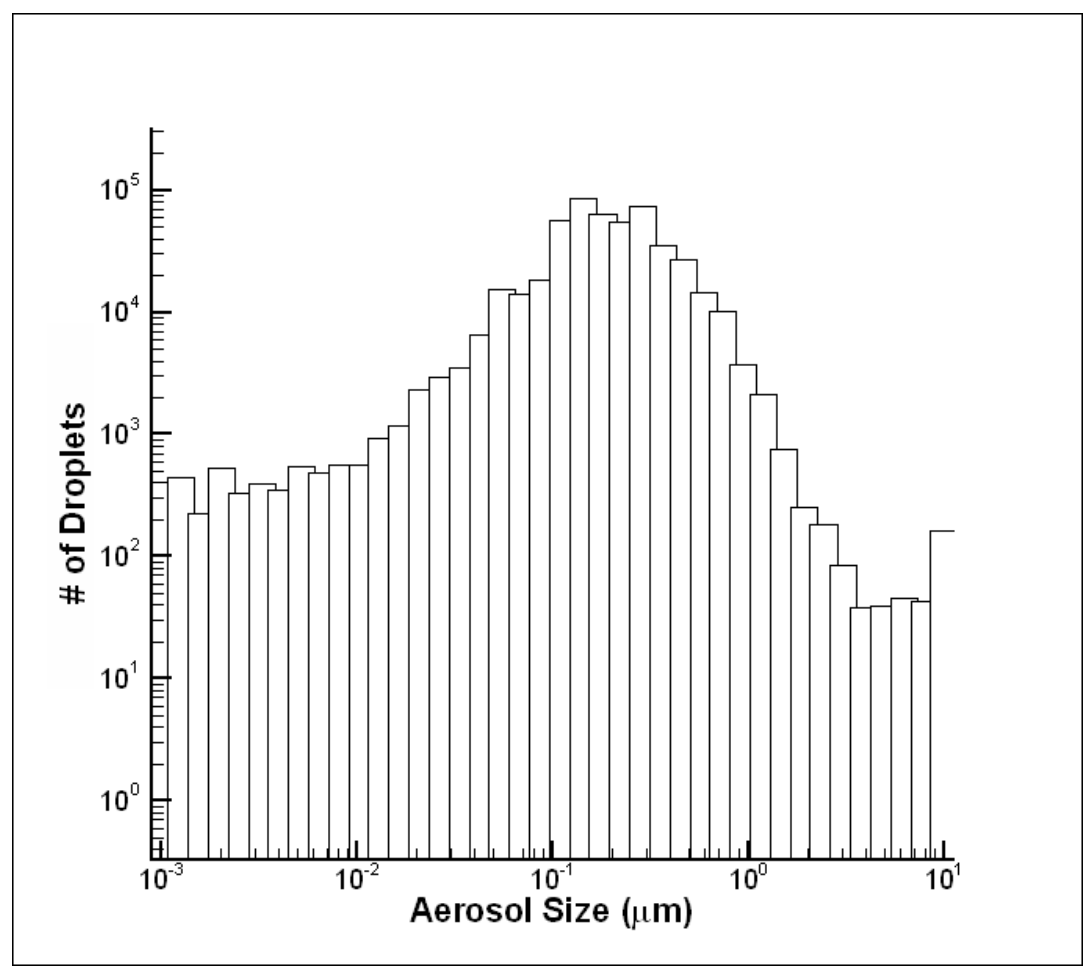

Figure 4.43: Accumulated aerosol size distribution after coughs (decreasing strength).

\subsection{Aerosol Generation during a Child's Cough}

The cough simulation has also been carried out in an anatomical pediatric upper airway. This model is generated based on the three dimensional scan of a child's cadaver [55]. The geometry of the upper respiratory tract for a child is given in Figure 4.44. The one dimensional geometry corresponding to this respiratory tract is also given in Figure 4.45. In this one dimensional representation the cross sectional area of the real geometry is calculated and an effective diameter is calculated to represent the actual geometry in an equivalent circular pipe.

For this geometry, the same coughing signal as in the other coughing cases with a lower magnitude is used. The magnitude of the pressure is 
decreased four times to match the flow rate of the cough. The variation of velocity in the axial direction at the peak of the cough and the variation of volumetric flowrate with time for the given boundary conditions are plotted in Figures 4.46 and 4.47 , respectively.
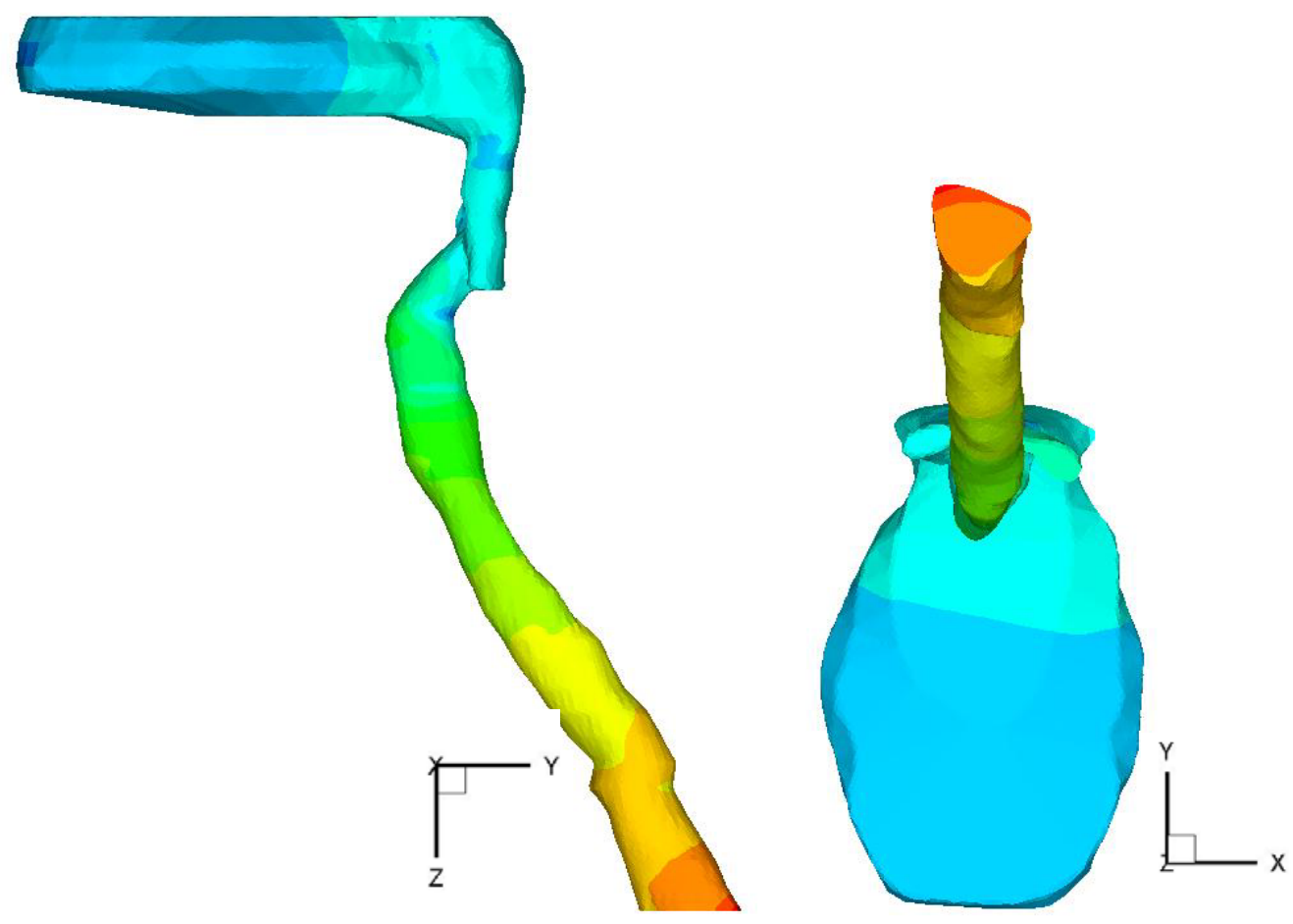

Figure 4.44: Upper respiratory tract of a child [55].

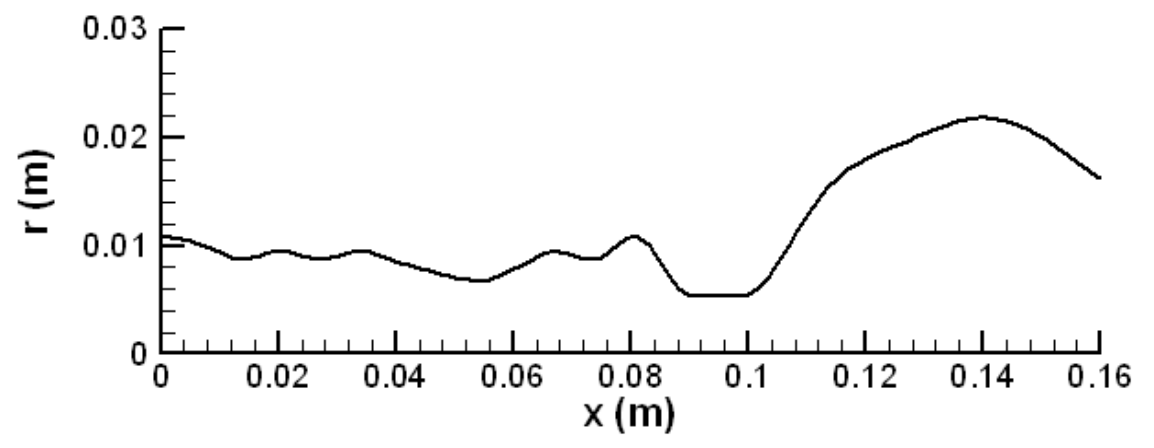

Figure 4.45: One-dimensional geometry for child's respiratory tract. 


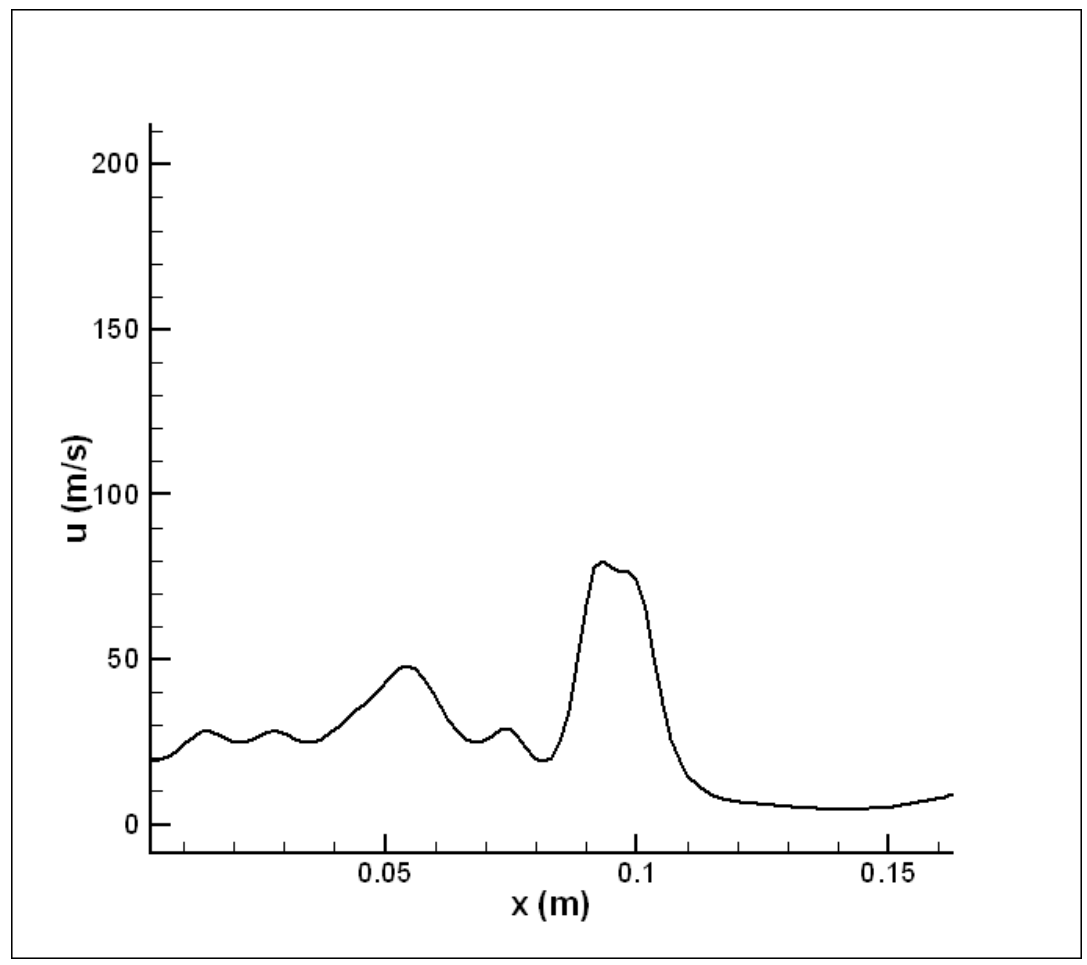

Figure 4.46: Axial velocity variation at the peak of the cough.

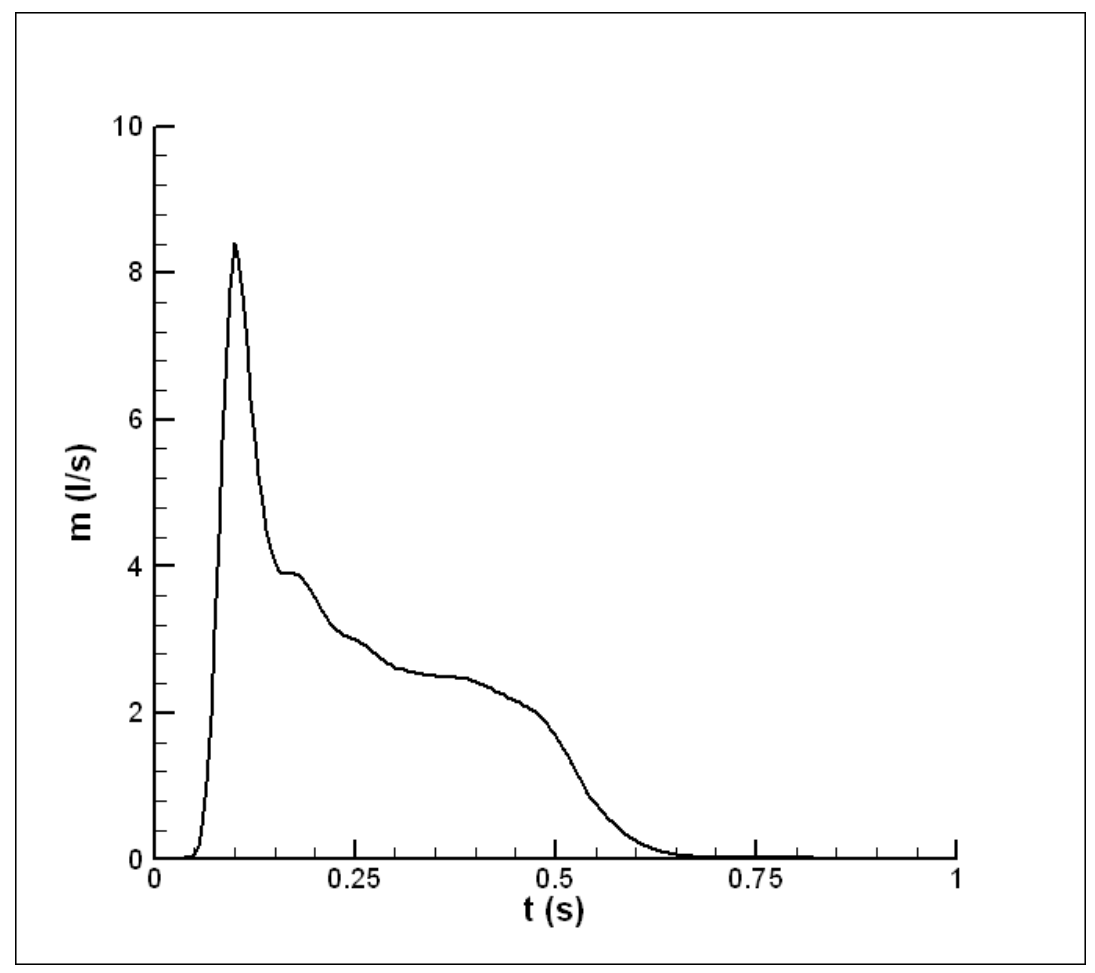

Figure 4.47: Variation of flow rate with time. 


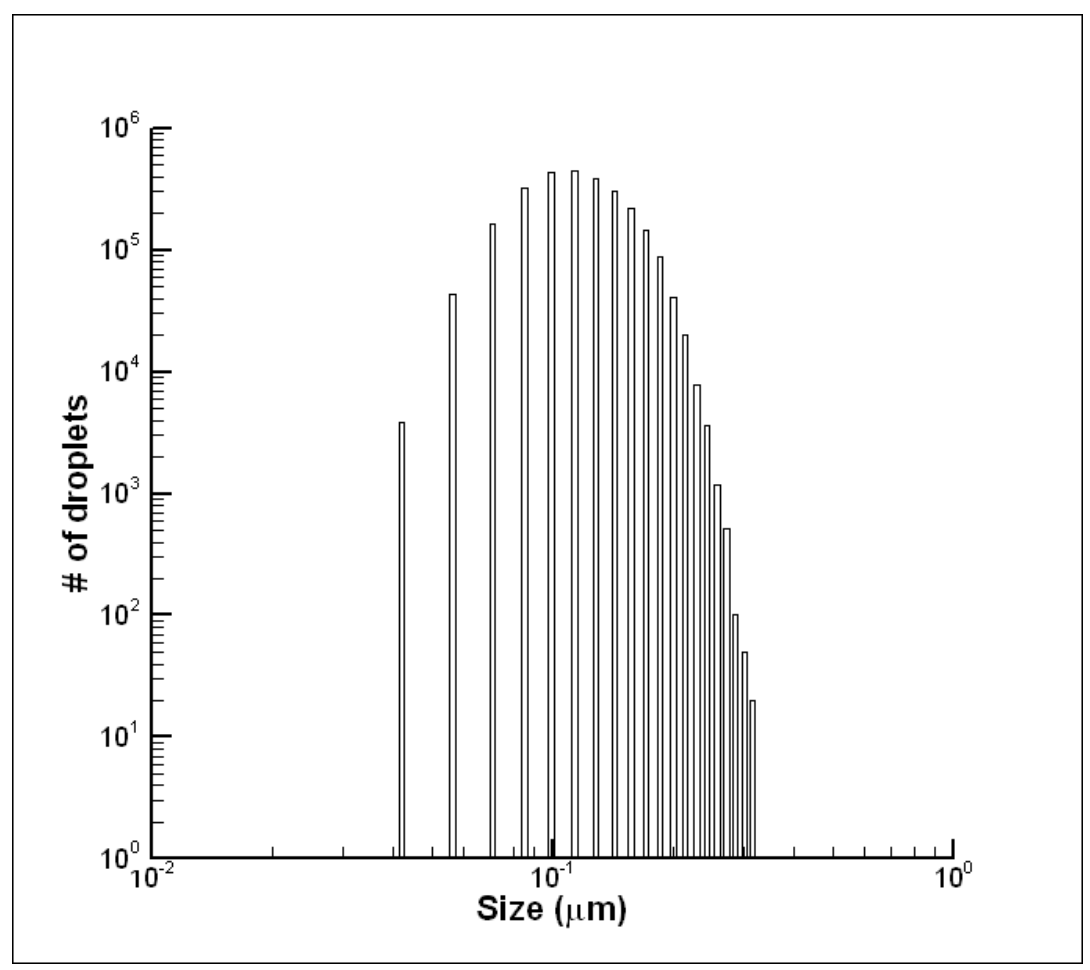

Figure 4.48: Averaged aerosol size distribution during a child's cough.

With the pediatric upper airway geometry and given conditions the aerosol distribution during the cough is obtained. It should be noted that except for the geometry and the boundary conditions all the other parameters, physical properties and model coefficients are kept constant in this child's cough simulation case. The aerosol size distribution is given in Figure 4.48. The cough simulation in this geometry provided a narrower range of aerosols than the previously used geometry and conditions. The total volume of the aerosols generated during the cough of the child is 0.062 $\mathrm{ml}$, which is composed of 2.6 million droplets, while during the adult's cough the total number of aerosols generated is 4.2 million droplets, which make up $0.087 \mathrm{ml}$. Although it is difficult to comment on the accuracy of the results obtained for pediatric upper airways, the trend of the size distribution curve is consistent with the previously obtained curves. The reason for a smaller maximum aerosol size can be explained by the smaller diameter of the trachea, which may cause smaller initial droplets to be entrained initially to 
start with. On the other hand the larger minimum droplet size can be explained by the lower peak velocity in the airways compared to the previously used geometry. The lower velocity leads to lower turbulent kinetic energy, which in turn leads to fewer breakup processes for the droplets. Though, it is not unexpected to obtain a different size distribution for a significantly different geometry, it is necessary to validate the model against experimental data before any definite conclusions can be drawn. 


\section{CHAPTER 5: \\ CONCLUSI ONS AND RECOMMENDATI ONS}

Optimist: "The glass is half full"

Pessimist: "The glass is half empty"

Engineer: "That glass is twice as large as it needs to be"

\subsection{Conclusions}

This research aims to develop a fairly simple yet an accurate model for the flow simulation as well as aerosol generation during coughing in the upper respiratory tract, mainly in the larynx, and the number and size distribution of the aerosols generated during coughing. This objective is important since some diseases, such as influenza, spread by dissemination and inhalation of aerosols of small droplet nuclei that are generated by coughing and remain airborne for an extended time. For that reason a better understanding of the generation of aerosols is important.

In this study aerosol generation, entrainment and breakup sub-models have been developed and integrated into the one-dimensional flow solver and successfully tested on some realistic cases. After tuning the model coefficients and using the available physical data, these models have provided consistent results with the experimental measurements.

In addition to the comparison with available experimental data, a parametric study has been performed to see the effect of physical 
parameters on the aerosol particle size distribution generated as a result of coughing.

Breathing and coughing conditions have been simulated successfully by PTM and FLUENT and the results are compared. It was also shown that the pseudo-two-dimensional model (PTM) produces acceptable results within a fraction of execution time of a multi-dimensional commercial CFD software FLUENT. The decrease in the total execution time when PTM is compared to the FLUENT, makes PTM a practical parametric study tool.

Moreover a preliminary study for particle deposition during breathing was conducted. During breathing simulations particles are injected into the system and their depositions patterns are obtained. The particle deposition patterns obtained within and in the vicinity of larynx agree with those found in the literature. These patterns may explain higher probability of cancer occurrence in the larynx.

\subsection{Recommendations}

With the aerosol generation and entrainment model developed in this study, the size distribution of the aerosols during cough can be calculated. This size distribution resulting from a cough may be used as the source term or as the boundary condition in investigation of the dispersion of the aerosol particles in a confined environment. The aerosol generation model can be used to represent the sick person's coughs and further models can be developed to track the dispersion of these aerosols in the confined room. 
Physical properties of the mucus should be studied further and the corresponding model parameters in the mathematical models should be refined. Model prediction will improve if the coefficients can be tuned against more detailed experimental data.

Including the physico-chemical interaction between the deposited particles, mucus and the respiratory tract should results in better physical representation. These in turn can be studied in more detailed to investigate the relationship between the particle deposition patterns and the occurrence of laryngeal cancer. 


\section{REFERENCES}

[1] Tatli, E., 2003, "One-Dimensional Transient Numerical Modeling of a Micro-Power Generation System," Master Thesis, West Virginia University, Morgantown, WV

[2] Celik, I.B., Ersahin, C., and Tatli, E., 2004, "GFS-1D: A One-Dimensional Transient Solver for Transport Phenomena," Technical Report, Mechanical and Aerospace Engineering Department, West Virginia University, Morgantown, WV, January, MAE-IC04-TR\#01

[3] Lindsley, W.G., 2005, Private Communication

[4] Frazer, D.G., 2005, Private Communication

[5] Afshari, A., Azadi, S., Ebeling, T., Badeau, A., Goldsmith, W.T., Weber, K.C., and Frazer, D.G., 2002, "Evaluation of cough using digital particle image velocimetry,". Proceedings of Second Joint EMBS-BMES Conference, 23-26 October, Houston, TX, IEEE, 975-976

[6] Goldsmith, W.T., Afshari, A., Azadi, S., Jones, B., and Frazer, D., 2001, "A system for analyzing aerosols produced by humans during respiratory maneuvers," Biomedical Engineering Society, Annual Fall Meeting, Durham, NC, BMES, S-141 
[7] Ersahin, C., Celik, I.B., Elci, O.C., Yavuz, I., Li, J., and Hu, G., 2004, "A simple model for fluid flow and particle motion inside the human larynx," Proceedings of 2004 ASME Heat Transfer/Fluids Engineering Summer Conference, Charlotte, North Carolina, July 11-15, HT-FED2004-56137

[8] Ersahin, C., Celik, I.B., and Yavuz, I., 2005, "Aerosol Generation and Deposition Patterns in the Larynx during Coughing," CFD Summit, Dearborn, MI, June 7-9

[9] Tucker, H.M., Ed., 1993, “The Larynx," 2nd ed. New York, Thieme Medical Publishers Co., 291

[10] Elci, O.C., 2004-2005, Private communication

[11] Staubesand, J., Ed., 1990, "Sobotta Atlas of Human Anatomy," Urban\&Schwarzenberg, Baltimore

[12] Elci, O.C., Dosemeci, M., and Blair, A., 2001, "Occupation and the risk of laryngeal cancer in Turkey," Scand J Work Environ Health, 27, 233-239

[13] Phalen, R.F., 1984, "Inhalation Studies: Foundations and Techniques," CRC Press Inc., Boca Raton, Florida

[14] Martonen, T.B., Zhang, Z., and Lessmann, R.C., 1993, "Fluid Dynamics of the Human Larynx and Upper Tracheobronchial Airways," Aerosol Science and Technology, 19, 133-156

[15] Katz, I.M., Davis, B.M., and Martonen T.B., 1999, "A numerical study of particle motion within the human larynx and trachea," J. Aerosol Sci., 30 (2), 173-183. 
[16] Katz, I.M., and Martonen, T.B., 1996, “Three-dimensional fluid particle trajectories in the human larynx and trachea," Journal of Aerosol Medicine, 9 (4) 513-520

[17] Elci, O.C., Akpinar-Elci, M., Dosemeci, M., Blair, A., 2002, "Occupational dust exposure and the risk of laryngeal cancer in Turkey," Scand J Work Environ Health, 28, 278-84

[18] Elci, O.C., Akpinar-Elci, M., Blair, A., Dosemeci, M., 2003, "The risk of laryngeal cancer by occupational chemical exposure in Turkey," JOEM, 45, 1100-1106

[19] Alipour, F., Scherer, R., and Knowles, J., 1996, "Velocity Distributions in Glottal Models," J ournal of Voice, 10 (1), 50-58

[20] Ertbruggen, C., Hirsch, C., and Paiva, M., 2005, "Anatomically Based Three-Dimensional Model of Airways to Simulate Flow and Particle Transport Using Computational Fluid Dynamics," J Appl Physiol, 98, 970-980

[21] Gemci, T., Corcoran, T.E., and Chigier, N., 2000, "Dispersion and Deposition of Inhalation Theraphy Sprays in the Larynx and Trachea Using Experimental and Numerical Methods," $8^{\text {th }}$ International Conference on Liquid Atomization and Spray Systems, Pasadena, CA, July

[22] Gemci, T., Corcoran, T.E., Yakut, T., Shortall, B., and Chigier, N., 2000, "Numerical Simulation of Particle and Air Velocity Fields in a Model of a Cadaver Throat," Private communication 
[23] Renotte, C., Bouffioux, V., and Wilquem, F., 2000, "Numerical 3D analysis of oscillatory flow in the time-varying laryngeal channel," Journal of Biomechanics, 33, 1637-1644

[24] Yavuz, I., Celik, I. and McMillian, M., 2002, "Knock Prediction in Reciprocating Gas-Engines using Detailed Chemical Kinetics," 2001 SAE Transactions, Journal of Engines, 110, Section 3, 981-987

[25] Celik, I., Yavuz, I., Smirnov, A., Smith, J., Amin, E., Gel. A., 2000, "Prediction of In-Cylinder Turbulence For IC Engines" Combustion Science and Technology, 150, 1-30

[26] Celik, I., Yavuz, I., Smirnov, A., 2001 "Large Eddy Simulations of InCylinder Turbulence for IC-Engines: A Review", Int. Journal of Engine Research, 2 (2), August

[27] Hinze, J.O., 1955, "Fundamentals of the Hyrdodynamic Mechanism of Splitting in Dispersion Processes," AIChE Journal, 1 (3), 289-295

[28] Narsiman, G., Gupta, J.P., and Ramkrishna, D., 1979, "A Model for Transitional Breakage Probability of Droplets in Agitated Lean LiquidLiquid Dispersion," Chemical Engineering Science, 34, 257-265

[29] Narsiman, G., Nejfelt, G., and Ramkrishna, D., 1984, "Breakage Functions for Droplets in Agitated Liquid-Liquid Dispersions," AlChE J ournal, 30 (3), 457-467

[30] Sathyagal, A.N., Ramkrishna, D., Narsiman, G., 1996, “Droplet Breakage in Stirred Dispersions. Breakage Functions from Experimental Drop-Size Distributions," 1996, 51 (9), 1377-1391 
[31] Martinez-Bazan, C., Montanes, J.L., and Lasheras, J.C., 1999, "On the Breakup of an Air Bubble Injected into a Fully Developed Turbulent Flow. Part 1. Breakup Frequency", J. Fluid Mech., 401, 157-182

[32] Martinez-Bazan, C., Montanes, J.L., and Lasheras, J.C., 1999, "On the Breakup of an Air Bubble Injected into a Fully Developed Turbulent Flow. Part 2. Size PDF of the Resulting Daughter Bubbles", J. Fluid Mech., 401, 183-207

[33] Shaffer, C.E., 2005, "Flow System Modeling with Applications to Fuel Cell Systems," MS Thesis, West Virginia University, Morgantown, WV

[34] MacCormack, R.W., 1982, "A Numerical Method for Solving the Equations of Compressible Viscous Flow," AIAA Journal, 20 (9), 12751281

[35] Anderson, D.A., Tannehill, J.C., Pletcher, R.H., 1984, Computational Fluid Mechanics and Heat Transfer, New York, McGraw-Hill

[36] McGuirk, J.J., and Page, G.J., 1990, Shock Capturing Using a PressureCorrection Method, AIAA J ournal, 28 (10), 1751-1757

[37] Gusheng, Hu., 2005, "Towards Large Eddy Simulation of Dispersed GasLiquid Two-Phase Turbulent Flows," Dissertation Thesis, West Virginia University, Morgantown, WV

[38] Ersahin, C., Celik, I., Yavuz, I., Lindsley, W., and Frazer D., 2006, "Cough Simulation by Means of a Pseudo-Two Dimensional Model," ASME Joint U.S.-European Fluids Engineering Summer Meeting, FEDSM2006-98100, Miami, FL, USA, July 17-20 
[39] Crowe, C., Sommerfeld, M., Tsuji, Y., 1997, "Multiphase Flows with Droplets and Particles," CRC Press, 0-8493-9469-4

[40] Vanoni, V. A. Editor, 1975 "Sediment Engineering," ASCE, New York, N.Y.,

[41] Celik, I.B., Rodi, W., 1991, "Suspended Sediment-Transport Capactiy for Open Channel Flow," J ournal of Hydraulic Engineering, 117 (2), 191-204

[42] White, F.M., 2003, "Fluid Mechanics," Fifth Ed., McGraw-Hill, 0-07240217-2, 293-340

[43] http://www. processassociates.com/process/dimen

[44] Lefebvre, A.H., 1989, "Atomization and Sprays," Hemisphere Publishing Corporation, New York

[45] Tsouris, C. and Tavlarides, L.L., 1994, "Breakage and Coalescence Models for Drops in Turbulent Dispersions," AIChE Journal, 40 (3), 395406

[46] Hesketh, R.P., Etchells, A.W., and Russell, T.W.F., 1991, "Bubble Breakage in Pipeline Flow," Chemical Engineering Science, 46 (1), 1-9

[47] Konno, M., Aoki, M., and Saito, S., 1983, "Scale Effect on Breakup Process in Liquid-Liquid Agitated Tanks," Journal of Chemical Engineering of Japan, 16 (4), 312-319

[48] Hu, G., 2004-2005, Private communications 
[49] Whisler, R., 2005, Private communications

[50] Badeau Jr., A. E., "A Droplet Formation and Entrainment Model for Stratified Liquid-Liquid Flows," Masters Thesis, West Virginia University, Morgantown, WV

[51] Li, J., 2005, "Critical Issues for Predicting Worker Exposure to Gaseous Contaminants in a Wind Tunnel," Dissertation, WVU, Morgantown, WV

[52] Lefebvre, A.H., 1989, "Atomization and Sprays," Hemisphere Publishing Corporation, New York

[53] Celik, I., Badeau, A., Kandil, S., Wilson, W., and Chang, P., 2005, "Modeling of droplet formation in stratified immiscible liquid-liquid flows," J ournal of Hydraulic Research, 43 (1), 86-97

[54] Kostoglu, M. and Karabelas, A.J., 1998, "On the attainment of steady state in turbulent pipe flow of dilute dispersions," Chemical Engineering Science, 53 (3), 505-513

[55] Allen, G.M., Shortall, B.P., Gemci, T., Corcoran, T.E. and Chigier, N.A., 2004, "Computational Simulations of Airflow in an In Vitro Model of the pediatric Upper Airways," Journal of Biomechanical Engineering, 126, 604-613 


\section{APPENDIX A}

\section{Particle Deposition during Breathing}

A preliminary study of particle deposition in the respiratory tract has been done as a starting point for a future study. In this study, first particles are introduced into the system to observe the trajectories of the particles and the distribution of the deposited particles. Particles are tracked by the use of subroutine developed by $\mathrm{Hu}[37,48]$. In this case the realistic boundary conditions (Sec. 4.2), have been applied at the subglottis (Fig. 4.14).

Quartz particles are injected into the system and are tracked, with a density of $2650 \mathrm{~kg} / \mathrm{s}$ in two different sizes, namely $1 \mu$ and $5 \mu$. A total number of 16500 particles are randomly injected at the inlet into the system and as soon as the particles have a contact with the wall of the larynx, they are assumed to deposit on the wall. The distribution of the particles on the wall is obtained in this way. This type of boundary condition is also not very representative since in the real life there exist physical interaction between the particles and walls, which would affect the efficiency of the particle deposition. However, as a preliminary study for a future work has been implemented here to show the capability of capturing the pseudo twodimensional flow field and producing acceptable results with those suggested in the literature. 
Distribution of the $1 \mu$ particles shows that, $55 \%$ of the particles are deposited on the larynx and $84 \%$ of the deposited particles deposit in the supraglottic area and $16 \%$ of them deposit in the subglottic area. This ratio slightly changes for $5 \mu$ particles and $50 \%$ of the particles are deposited on the larynx and $89 \%$ of the deposited particles deposit supraglottic area and $11 \%$ of them deposit the subglottic area.

The deposited particle distribution is also investigated in several layers of the larynx as shown in Figure A.1 and the particle distribution among these layers are plotted as normalized histograms in Figure A.2. These results were compared with the numerical study carried out by Katz et al. [15] for inspiratory flow conditions, which is actually too restrictive as particles is transported back as shown in our study. Nevertheless results obtained by the present study are found to be in good agreement with their study. The details of this study can be found elsewhere, Ersahin et al. [7].

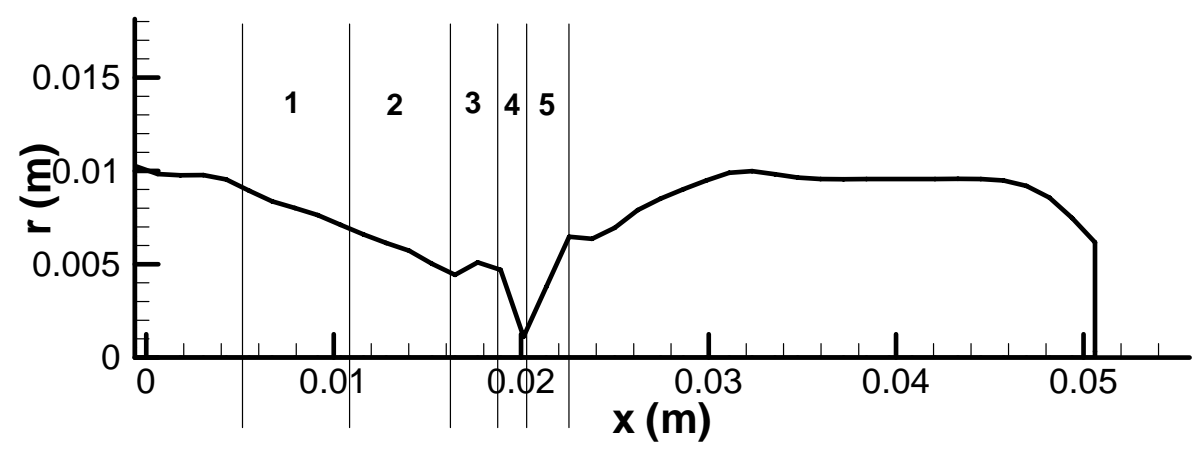

Figure A.1: Layers of the larynx. 


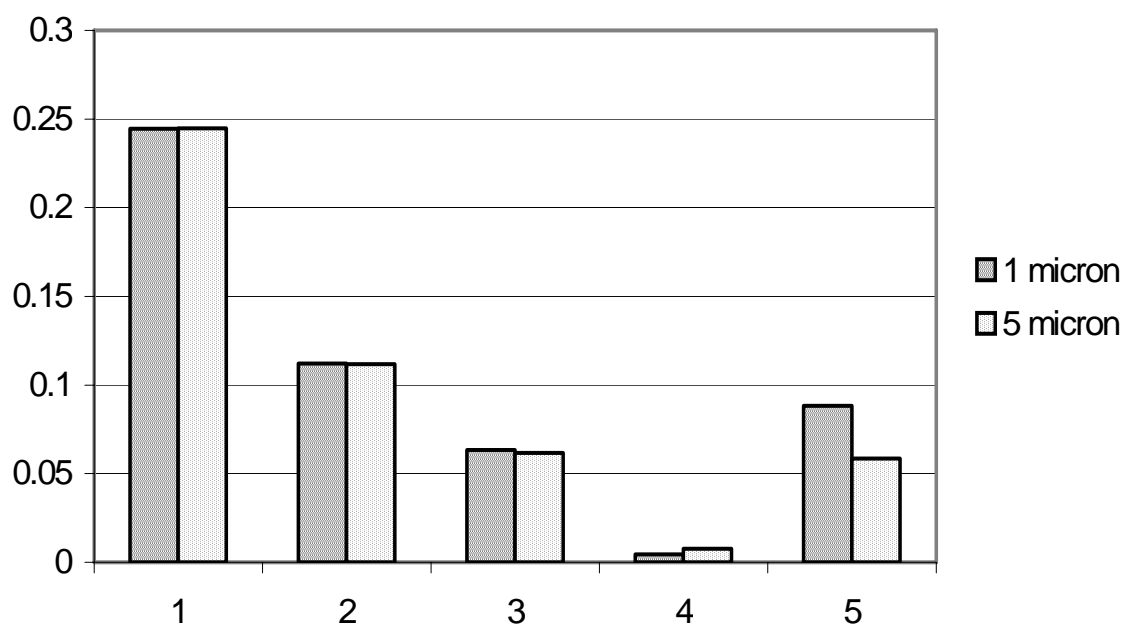

Figure A.2: Distribution of deposited particles among the sub-regions.

As it can be seen from Figure A.2, in both cases the particles give similar distribution. The reason for this similar distribution is that the Stokes number for both particle sizes is above or close to 1 . Stokes number is defined as the ratio of the particle relaxation time to the inertial time scale. Inertial time scale is calculated by dividing the characteristic length scale of the flow by the velocity scale, and the particle relaxation time is defined as $\rho d_{p}^{2} / 18 \mu$. For cases, where the Stokes number is larger than 1, particles will continue in a straight line as the fluid turns around the obstacles. The inertial time scale of particles is found to be $2.90 \times 10^{-5} \mathrm{~s}$. The corresponding particle relaxation time scales are $1.47 \times 10^{-5}$ and $3.68 \times 10^{-4} \mathrm{~s}$ for $1 \mu$ and $5 \mu$ particles, respectively. These values leads to $\mathrm{St}=12$ for $5 \mu$ and $\mathrm{St}=0.5$ for $1 \mu$ particles. The time scale explains why there is very little difference between the deposition patterns of small and larger particles. The expected difference between dispersion and deposition of different size particle could only be detected when there is sufficient time for the particles to adapt to the local fluid motion. Another factor in seemingly atypical results could be the large deposition surface intervals selected for the histogram. If these intervals were smaller one could possibly see a shift in maximum deposition area for different size particles. It should also be mentioned that resolution of 
fine scale fluid turbulence was not adequate in this pseudo-one dimensional fluid model. It seems that the random turbulent fluctuations that will effect primarily the fine particles need to be incorporated in the model e.g. via isotropic assumption $u_{\text {fluid }}(t)=\bar{u}_{\text {fluid }}+\left(u_{r m s}\right)_{\text {fluid }}$. 


\section{APPENDIX B}

\section{Sensitivity Analysis for Entrainment Model}

The sediment discharge in rivers can be calculated by measurement of discharge at certain time intervals and plotting these values against the total fluid discharge and a best line can be fit to these experimental measurements and the resulting curve is called the sediment transport curve. An example for this curve is given in Figure A.3, where the sediment discharge of Colarado river is plotted against the water discharge.

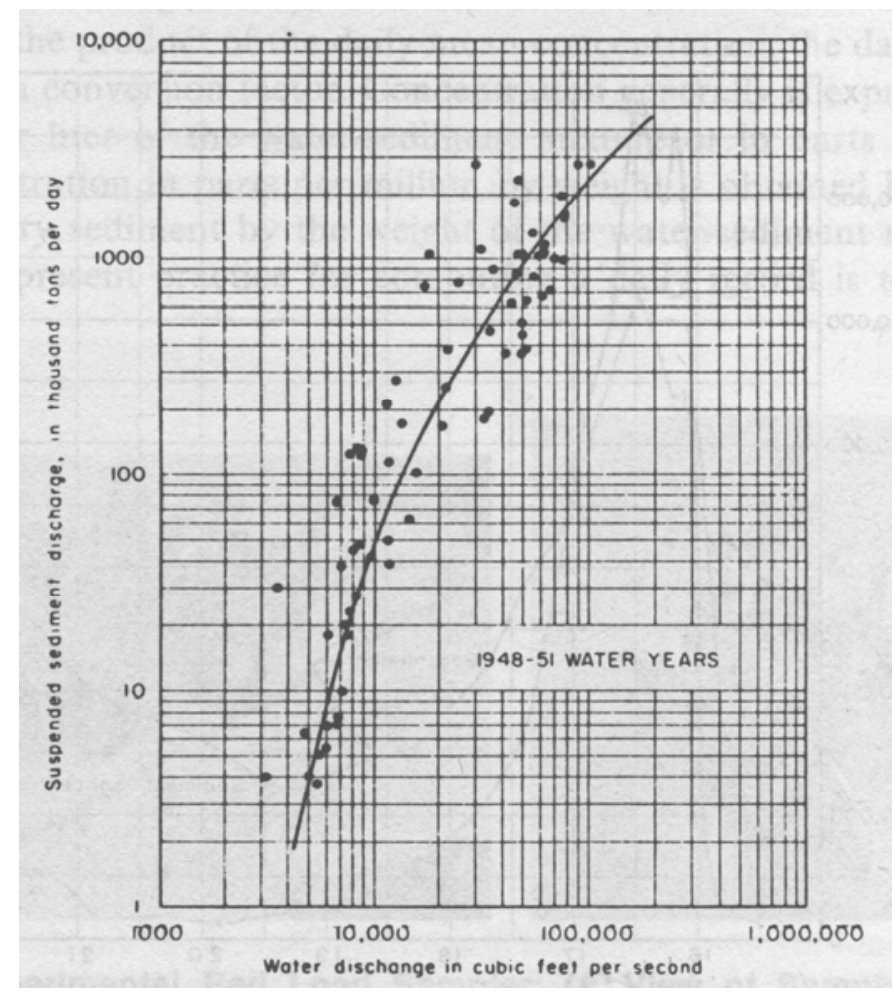

Figure A.3: Sediment transport curve, Colorado River, Arizona [40]. 
An analogy to sediment discharge in rivers can be applied to the total discharge of mucus during a cough in the upper respiratory tract. For this purpose, the total entrainment can be plotted against the velocity of the air flow through the trachea. In this approach, the entrainment of mucus is equivalent to the sediment discharge in the river and the velocity can be taken as the flowrate by assuming constant area of the trachea and constant density of the fluid, which in turn, gives the same trend as the flowrate with a constant factor of area of the trachea times the density of the fluid. For this plot, a range of velocity is selected and the shear stresses at these velocities are calculated. The shear stress is plugged into the entrainment equation (Eqn. 3-17) and the total entrainment is calculated.

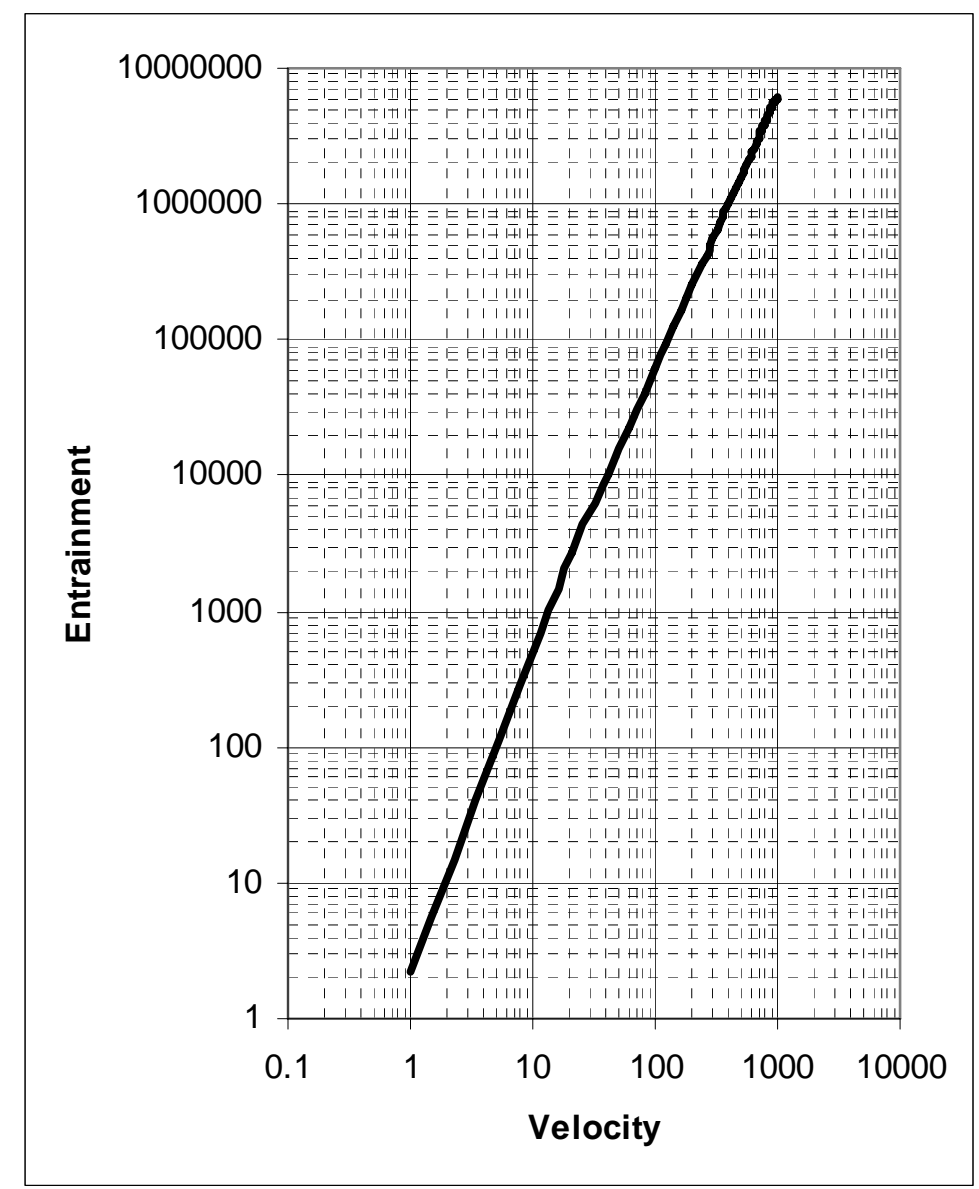

Figure A.4: Total entrainment versus velocity in the trachea. 
As it is seen in Figure A.4, the total entrainment calculated by the model equation (Eqn. 3-17) gives a similar trend as in the experimentally obtained sediment discharge curve (Fig. A.3). This approach shows that, the trend of the entrainment amount calculated by the proposed model agrees with the experimentally obtained sediment discharge qualitatively. For a quantitative comparison, measurements in a setup representing the upper respiratory tract covered with mucus are required. 


\section{APPENDIX C}

\section{Manual for the Solver}

\section{I ntroduction}

This code is based on previously developed one-dimensional lumped model. The details of this one-dimensional solver can be found elsewhere [2]. The previously developed Fortran77 code is translated into Fortran90 subroutine, so that this solver can be used in conjunction with other subroutines easily. All the equations and the method is kept the same during the translation. The main purpose for this translation is to be able to develop a more modular and easy to handle program. With this approach, by including or excluding particular modules, the program can easily be used just to solve a one-dimensional flow or all the other features can be included.

\section{Director Structure}

Two subfolders are required in the running directory of the program. One of them is input and the other one is output. Input files required for the run should be located in the input directory and the output files will be created (or replaced, if there are any) in the output directory. 


\section{I nput Files}

There are four input files required and must exist in the input directory for a successful execution. These are ASClI files which can be opened and edited bye any text editor. However, extra precaution should be used while editing these files. Any unintentional change in this file may cause a run time error which prevents the program running as expected.

Input.dat is the main input file, which keeps the run parameters for the code. Type of run (restart or not), number of iterations, time step, etc. are kept in this file. This file may be updated as necessary by keeping the exact same format. There may be more than one value on each line. In this case only the first value will be taken into consideration and the rest will be ignored. On each line after the value itself, the variable name as used in the program and a brief explanation is provided for clarity. Number of blank lines and comment lines should be kept as they are. Adding or removing even a blank line would cause a run time error or a wrong value to be taken as an input.

Physical.dat file is where the physical properties of the fluid are kept. If any physical property should be changed, the modification should be done in this file. Many physical.dat files can be kept in the same folder with extension of the name of the fluid, and can be replaced by input.dat for a quick change of the fluid in the system. The original library of the fluids in the input folder includes water and air.

Third one is the curve. dat file. This file contains the radius variation of the geometry along the axial direction. It has two columns, left being the axial direction and right column is the radius at that axial location. The number of data in this file should match the nxmax variable in Parameters module. If the data number is more than nxmax, the remaining lines are 
ignored. If nxmax is larger than the number of data in this file, it causes a run time error and the program halts.

The last input file is the PGEM.dat file. This file holds the model coefficient and physical properties for the aerosol generation and entrainment model. The modification can be done as in the other two files. The physical properties, density, surface tension etc, are for the dispersed phase. This is different from the physical.dat file.

\section{Output Files}

All the output files are written to output folder. There are many output files already created by default. The number of files can be increased and decreased by modifying the Printout subroutine in the auxiliary module of the code. Most of these output files are for transient files and there are some for the final converged solution. Please note that existing of final files does not guaranteed a converge solution. Convergence of a solution is the responsibility of the user. The frequency of the printout can be adjusted in the input file with the parameters number of frames. The default value is 200 and regardless of the iteration number (assuming iteration number larger than 200) 200 transient results are written out. Therefore, the frequency of the print out is iteration number/200. The output files are written in Tecplot format by default.

\section{Layout Files}

For each output file created by default, there exists a layout file. These layout files are kept in the layouts folder. Opening any layout files opens the related output file from the output folder and sets the predefined layout 
scheme for this particular file. Although this folder is not required for the program to execute successfully, it is highly recommended that all the layouts files are kept in layout folder for quick access and simplicity of each subfolder and the main run folder.

\section{Restart Files}

Depending on the restart parameter in the input file, a restart file is created at the end of the run. This restart file keeps all the data required for continuation form that time step. Usually it is a good idea to keep a restart file handy, since sometimes it may take too long for a flow to reach steady state. You can always change the flow parameters, but restarting from a restart file usually accelerates the convergence rate. The restart file is not as user friendly as the input files, therefore editing a restart file should be avoided. If it is necessary, one can match the variable names in the write_out subroutine in auxiliary module and the restart file and update the required values.

\section{Terminating a Run}

Usually the run executes until the user defined iteration level is reached and quits running. In some occasion it may be necessary to stop a run before the maximum iteration number is reached. You can simply use Control- $\mathrm{C}$ for a hard stop. However, in this situation you loose all the data you have obtained so far for that particular run. Also since the output files are replaced once a new run is started, the output files may not contain the full information once the run is terminated. If you like to stop a run without loosing any information, open the stop.dat file in the input folder and replace the letter F (False) with $T$ (True). Save this file and close it. At the next check point of the run, this file will be read and the execution will terminate smoothly as it reached the maximum iteration level. The stop check is done 
every time the output files are updated. Therefore it is checked 200 times during a run. It may be checked more occasionally but this approach affects the speed of the execution.

\section{Flow Structure}

The main code is called the PTLFlow. This is the coordinating main program. It calls all the other subroutines.

\section{Modules and Subroutines}

There a several modules, which holds the relevant subroutines together. This improves the modularity of the code. It is very easy to include or exclude individual modules depending on the results needed.

mod_Auxilary: Includes all the subroutines required for main program to communicate with the user and also sets up the initial conditions for the flow.

mod_Boundary: Includes the subroutine to set the boundary conditions for coughing case.

mod_Cough: Includes to subroutines, to select a random cough signal and apply the corresponding boundary condition.

mod_OneD: This module includes all the subroutine for the solution of one dimensional flow. The subroutines in this module were translated the original one-dimensional code (GFS-1D) into Fortran90. 
mod_Parameters: Keeps the global variables for maximum array declarations.

mod_PBM: Includes the subroutines related with particle breakup process.

mod_PGEM: Includes the subroutines related with particle entrainment model.

mod_precision: A parameter file to set up the precision level for the entire program. The code can easly be run as a single or a double precision by modifying this module.

mod_random: Includes the generic pseudo random generation subroutines. It is used to randomly select the cough signal and also randomly slect droplet size during the breakup process.

mod_SetBC: Includes the subroutines to set the boundary conditions.

mod_TDMA: Includes TDMA solver.

mod_TKE: Includes the subroutine to solve the integral turbulent kinetic energy equation.

mod_TwoD: Includes subroutines to generate the two-dimensional flow field.

mod_variables: Includes all the global variables used commonly in all the subroutines. 


\section{Variables}

All global variables are defined in the mod_Variables module. These variables are accessible from each subroutine, which includes the mod_Variables module. This eliminates the passing of commonly used variables between the subroutines and also decreases the memory usage by using only one memory location for these variables. All local variables used within a particular subroutine are defined within the subroutine. 